\title{
3 Regulieren
}

Vor dem Einstieg ins Regulierungkapitel erfolgt im Rahmen eines Exkurses die exemplarische Besprechnung einer paradigmatischen Regulierungsmaßnahme. Der Fokus liegt hier auf einem selbstregulatorischen Ansatz. Als solche werden Lenkungsmaßnahmen bezeichnet, die von regierungsexternen Akteuren wie Herstellern oder Umweltorganisationen ergriffen und beispielsweise in Form einer Verhaltensanweisung oder eines Risikomanagementschemas publiziert werden. Dieses Format stellt im Regulierungsdiskurs der Nanotechnologie eine verbreitete Praxis dar. Im Zentrum des nachfolgenden Exkurses steht dabei die in den Vereinigten Staaten aus der Zusammenarbeit zwischen dem multinationalen Unternehmen DuPont und der Umweltorganisation Environmental Defense Fund entwickelte Handlungsanweisung Nano Risk Framework. Daran soll gezeigt werden, wie eine solche Maßnahme inhaltlich aussieht und mit welchen Zielen und Absichten eine solche ergriffen wird. Ebenso soll aufgezeigt werden, wie selbstregulatorische Maßnahmen zu der für den Regulierungsdiskurs der Nanotechnologie paradigmatischen Delegation der Regulierungsverantwortung an die Gesamtgsellschaft beitragen. Dazu werden im nachfolgenden Exkurs die in der Maßnahme vorgesehenen Regulierungsaspekte, die damit verknüpften Praktiken und ihre thematische Orientierung aufgezeigt.

Exkurs: Das EDF - DuPont Nano Risk Framework

Im Jahr 2007 publizierten die Umweltorganisation Environmental Defense Fund (EDF) und das multinationale Chemieunternehmen DuPont in einem gemeinsamen Raster für das Risikomanagement von Nanomaterialien; das Nano Risk Framework (NRF). Das Ziel der beiden Organisationen lag darin, für Hersteller und Vertriebsorganisationen einen umfassenden Risikoanalyse- und Risikomanagementprozess zu entwickeln, damit diese potenzielle Risiken von Nanomaterialien professionell behandeln. Das Schema umfasst sechs detaillierte Schritte für die Bearbeitung von Schlüsselaspekten und schlägt Risikomanagementpraktiken vor. Zudem beinhaltete das Schema eine Deklaration der anwendenden Organisation, sich an freiwilligen Regierungsprogrammen in diesem Bereich zu beteiligen (Medley \& Walsh 2007).

Die Erarbeitung des Nano Risk Framework erfolgte in beiden Organisationen auf der obersten Führungsebene. Im Vorfeld hielten die beiden Firmenchefs ihr Bekenntnis zur Zusammenarbeit im Bereich der Nanotechnologie in einem gemeinsamen Zeitungsartikel fest (Krupp \& Holliday 2005). Die beiden Organisationen setzten eine 
gemeinsame Arbeitsgruppe ein, in welcher das Schema kooperativ erarbeitet wurde. Das Nano Risk Framework wurde flexibel aufgebaut. Es orientierte sich explizit am Vorsorgeprinzip und schloß in seiner Entwicklungsphase auch externe Expertise ein. Dies geschah einerseits durch die Präsentation der Maßnahme in öffentlichen Veranstaltungen in verschiedenen US-amerikanischen Städten und andererseits mittels eines Aufrufs auf der Projektwebsite, der Kommentare und Ergänzungen erbat. In seiner Einleitung betonten die Autorinnen und Autoren, dass das Schema nicht auf Annahmen, sondern auf Daten zur Gefährlichkeit bzw. zur Sicherheit von bestimmten Materialien basieren und dass bei der Abwesenheit solcher Informationen von einem sogenannt ,vernünftigen worst-case Szenario' bzw. einem Analogieschluss mit verwandten Materialien ausgegangen werden sollte. ${ }^{80}$

Das Ziel der lancierenden Organisationen lag nicht in einer prinzipienbasierten Verhaltensanweisung, wie einem Ethikkodex. Vielmehr sollte mit dem handlungsorientierten Schema ein Werkzeug bereitgestellt werden, mit welchem Anwenderinnen und Anwender bestimmter Materialien Sicherheitsinformationen sammeln, dokumentieren und kommunizieren können. Ebenso sollte ihnen das Instrument die Deklaration unvollständiger Informationen, den Umgang mit Informationslücken und die Begründung eigener Risikomanagemententscheidungen und -handlungen erleichtern (Medley \& Walsh 2007). Das Schema umfasste 101 Seiten Anweisungen zur korrekten Durchführung einer Risikoanalyse spezifischer Nanomaterialien. Diese wurde in sechs Schritte unterteilt und behinhaltete zusätzlich 14 Seiten Arbeitsblätter mit einer Klassierung von nanoskaligen Chemikalien hinsichtlich unterschiedlichster Eigenschaften und Formen. Das Schema wurde auf eine iterative Verwendung hin aufgebaut und umfasst die folgenden sechs Schritte (ibid. 8-10):

1. Schritt: Beschreibung der verwendeten Materialien und ihrer Anwendungen.

2. Schritt: Analysen der physikalischen und chemischen Materialeigenschaften, der inhärenten Umwelt-, Gesundheits-, und Sicherheitsgefährdungen und der damit verbundenen beabsichtigten und unbeabsichtigten Expositionen von Mensch und Umwelt über den gesamten Lebenszyklus.

3. Schritt: Bewertung der in den Materialprofilen gesammelten Informationen zur Identifikation und zur Charakterisierung der material- und anwendungsspezifischen Risiken hinsichtlich ihrer Beschaffenheit, des Ausmaßes und der Eintretenswahrscheinlichkeit. Feststellen und Priorisierung von Lücken in den Lebenszyklusprofilen und Entscheidung über den Umgang mit diesen Lücken.

4. Schritt: Evaluation der verfügbaren Optionen und Entwicklung von Handlungsanweisungen im Umgang mit den festgestellten Risiken, wie beispielsweise Kontrollen, Schutzausrüstung, Risikokommunikation und Anpassungen beim Prozess oder beim Produkt.

5. Schritt: Je nach Produktentwicklungsstufe erfolgende Beratung durch das zuständige Begutachtungsteam und Entscheidung, ob und in welcher Kapazität die Ent-

80 Siehe http://www.nanoriskframework.com/ (5.12.2012). 
wicklung und die Produktion fortgesetzt werden sollten. Dokumentation der Entscheidung und Begründung durch den Anwender in einem transparenten Entscheidungsfindungsprozess und Austausch der relevanten Informationen mit internen und externen Anspruchsgruppen. Bei Bedarf nach zusätzlichen Informationen erfolgen weitere erforderliche Schritte. Entscheidung über Zeitplan und Bedingungen zukünftiger Aktualisierungen der Risikoanalyse gemäß Arbeitsblatt.

6. Schritt: Revision und Aktualisierung der Risikoanalyse durch wiederholt angesetzte gezielte Bewertungen durch den Anwender. Sicherstellung, dass die Risikomanagementsysteme wie geplant durchgeführt und die Systeme angesichts neuer Informationen, Daten und Voraussetzungen, wie neuer Expositionsmuster angepasst werden. Information relevanter Anspruchsgruppen über diese Änderungen.

Die lancierenden Organisationen EDF und DuPont verbanden mit ihrer Initiative die Absicht, dass sich das Nano Risk Framework zu einem internationalen Standard entwickelt. Ihr Ziel lag darin, dass die verschiedenen Hersteller diesen Standard anwenden und bei Vorliegen neuer Informationen gemeinsam weiterentwickeln und verbessern.

Nach der Lancierung des Nano Risk Framework im Jahr 2007 führten EDF und DuPont zwei interaktive Anlässe in San Francisco und in Boston durch, in welchen sie das Schema interessierten Anspruchsgruppen und der Öffentlichkeit vorführten. Nach seiner Publikation wurde der Ansatz von unterschiedlichen Seiten als zu komplex und zu aufwändig kritisiert. Zudem verfassten verschiedene Nichtregierungsorganisationen eine gemeinsame Erklärung, in welcher sie das NRF als grundlegend falschen Ansatz bezeichneten, mit den drängenden Fragen der Nanotechnologie umzugehen und eine robuste gesetzliche Regulierung forderten. Ebenfalls wurde bezweifelt, ob das Schema den Bedürfnissen und Perspektiven der Hersteller, insbesondere kleinerer Unternehmen und Neugründungen gerecht werden kann (Maynard 2011). Im Jahr 2011 übernahm die Internationale Standardorganisation ISO ein an das Nano Risk Framework angelehntes Schema in ihrem technischen Bericht über die Risikoevaluation von Nanomaterialien (ISO 2011). Auch wenn sich die in verschiedenen Publikationen zum NRF formulierten Ziele, dass das Schema von weiteren Firmen übernommen wird und sich branchenweit als Standard etabliert, als zu hoch herausgestellt haben (IRGC 2009), haben Teile des Schemas in eine branchenübergreifende ISO Empfehlung gemündet (Maynard 2011).

Das Fallbeispiel der vom Unternehmen DuPont und der Umweltorganisation Environmental Defense Fund entwickelten Handlungsanweisung $\mathrm{Na}$ no Risk Framework zeigt ein grundsätzliches Problem der Selbstregulierung auf. Da sie außerhalb der traditionell für die Regulierung zuständigen staatlichen Institutionen entsteht, bleiben zentrale Aspekte unklar; nämlich die Frage nach ihrer Festlegung, ihrem Geltungsbereich und damit auch nach ihrer Durchsetzung und ihrer Wirkung. 


\subsection{Regulieren in Nichtwissensdiskursen}

Im Zentrum dieses Kapitels steht das Regulieren als eine Strategie des Umgangs mit dem mit der Nanotechnologie verbundenen Nichtwissen. Dabei stehen zwei Leitfragen im Fokus dieser Analyse: Die erste fragt nach der Behandlung von Nanomaterialien in der Umweltgesetzgebung und die zweite betrifft die von unterschiedlichen gesellschaftlichen Akteuren ergriffenen selbstregulatorischen und freiwilligen Maßnahmen. Diese Fragen werden länderspezifisch analysiert. Hier zeigt es sich, dass Regulierungsdiskurse neben der nationalen- und der supranationalen Ebene der Europäischen Union auch zunehmend in internationalen Foren stattfinden, wie beispielsweise in internationalen Standardorganisationen wie ISO oder in den nanotechnologiespezifischen Arbeitsgruppen der OECD, in welchen nationale Behördenmitglieder kooperieren.

Die vorliegende Untersuchung postuliert ein breites Verständnis des Regulierungsbegriffs. Dieser umfasst nicht nur Praktiken wie die gesetzliche Regulierung, sondern ebenso unterschiedliche formelle und informelle Steuerungs- und Lenkungsinstrumente von Regierungsorganisationen. Ebenso berücksichtigt werden freiwillige und selbstregulatorische Ansätze von regierungsexternen Anspruchsgruppen (siehe Kapitel 1.2). Der Begriff des Regulierens meint hier also die unter dem Begriff des ,harten Rechts' (hard law) aufgefassten rechtlich bindenden Ansätze und Statute, wie Gesetze und Verordnungen. Ebenso umfasst der Regulierungsbegriff hier die als ,weiches Recht' (soft law) bezeichneten quasi-legalen Instrumente, welche keine rechtlich bindende Kraft haben oder deren bindende Kraft schwächer ist als traditionelles gesetzliches Recht. ${ }^{81}$ Solche Instrumente umfassen hier behördlich verordnete Verhaltensanweisungen mit quasi-verbindlichem Charakter, also freiwillige, nicht bindende Regulierungsansätze von Regierungen auf nationaler Ebene sowie die freiwilligen Datenmeldeschemen (Voluntary Reporting Scheme for Engineered Nanoscale Materials, VRS) der britischen und das (Nanomaterial Stewardship Program, NMSP) der US-amerikanischen Umweltbehörde. Auf supranationaler Ebene der Europäischen Union beinhalten freiwillige Regulierungsmaßnahmen beispielsweise Mitteilungen der Kommission, wie dieje-

81 Ursprünglich wird der Begriff „soft law“ hauptsächlich für internationales Recht benutzt. In der Zwischenzeit wird er jedoch auch auf andere Bereiche des inländischen Rechtes übertragen, wie freiwillige Regulierungsansätze durch Behörden (siehe z.B. Kirton \& Treblicock 2004; Nasser 2008). 
nige zum Verhaltenskodex für verantwortungsvolle nanowissenschaftliche und nanotechnologische Forschung und die Empfehlung der Europäischen Kommission zur Definition von Nanomaterialien (COM 2008a; COM 2011).

In diesem Verständnis beschränkt sich das Regulieren also nicht nur auf gesetzliche Interventionen, sondern umfasst auch freiwillige ,eingrenzende und risikominimierende' wie auch ,fördernde und ermöglichende' Maßnahmen von Regierungsakteuren (Bora 2012, 352). Ebenfalls unter dem Regulierungsbegriff subsumiert werden hier zudem von nichtstaatlichen Akteuren ergriffene Maßnahmen, die sich oft an der Schnittstelle zwischen Wissenschaft, Politik Wirtschaft und Öffentlichkeit entwickeln. Solche durch externe Akteure ergriffene Regulierungsmaßnahmen werden auch als selbstregulatorische Steuerungs- und Lenkungsansätze bezeichnet. Sie werden in prinzipienbasierte, wie beispielsweise ethisch geprägte $^{82}$ und handlungsorientierte Ansätze unterschieden. Letztere umfassen die konkreten Handlungsanweisungen und Sicherheitsbestimmungen, Leit- und Richtlinien, wie beispielsweise die vom Branchenverband der chemischen Industrie (VCI) mit Herstellern entwickelten Schemen. ${ }^{83}$ Ebenfalls als selbstregulatorische Ansätze aufgefasst werden die Festlegung von Definitionen, Standards und Risikomanagementansätzen mit dem Ziel, einen übergreifenden, allgemeingültigen Ansatz zu lancieren, wie der vom multinationalen Chemikalienhersteller DuPont und der Umweltorganisation Environmental Defense Fund entwickelte handlungsorientierte Verhaltenskodex, das Nanoriskframework (siehe Einleitung Kapitel 3). Somit nehmen im Regulierungsdiskurs nicht alleine Akteure aus Po-

82 Die ethische Prägung meint normativ unterliegende Ansprüche wie Verantwortung oder Nachhaltigkeit (Kaiser, et al. 2009).

83 Weitere Beispiele umfassen die Leitlinien und den Code of Conduct des multinationalen Chemieunternehmens BASF und des Verbandes der Chemischen Industrie Deutschlands (VCI), Leitfäden und Sicherheitsdatenblätter von VCI und der deutschen Bundesanstalt für Arbeitsschutz und Arbeitsmedizin (BAuA), der Code of Good Practice des multinationalen Chemieunternehmens Bayer, der VCI und International Council for Chemical Associations (ICAA) Responsible Care Global Charter, Risikomanagementansätze des multinationalen Chemiekonzerns Evonik und Laborsicherheitsrichtlinien der Fachgesellschaft American Chemical Society (ACS) und des Branchenverbandes American Chemistry Council (ACC). Für Verhaltenskodizes im Bereich der Nanotechnologie siehe z.B. (Bayer 2007; COM 2008a; NIA 2008). Für eine Analyse solcher Nanokodizes, siehe (Bowman \& Hodge 2009). 
litik und Verwaltung teil, sondern ebenso unterschiedlichste gesellschaftliche Anspruchsgruppen aus Wissenschaft (Forschung und Forschungspolitik), Industrie, Dienstleistung, Branchen- und weiteren Verbänden, Denkfabriken (Think Tanks) und Umwelt- und Konsumentenschutzorganisationen (vgl. z.B. Boekaerts 2005), die ihre Maßnahmen individuell oder kooperativ entwickeln. Reguliert wird also innerhalb und außerhalb staatlicher und staatenübergreifender Regierungsorganisationen und -institutionen.

Im Regulierungsdiskurs verhandelte Themen umfassen zentral die Frage nach dem Regulierungsgegenstand, dem Regulierungsbedarf und den Lücken der aktuellen Regulierung bezüglich der Behandlung der Nanotechnologie und hierin insbesondere der Nanomaterialien. Dabei akzentuiert sich der zentrale Konflikt des Regulierungsdiskurses an der Frage nach der Neuartigkeit bzw. der Herkömmlichkeit der Nanotechnologie und damit verknüpft nach dem Erfordernis neuer Regulierungen bzw. der Zuständigkeit der bestehenden Gesetzgebung. Dabei lässt sich im Diskursverlauf eine Verengung des thematischen Fokus auf Nanomaterialien und darin hauptsächlich auf die Bereiche Umwelt, Gesundheit und Sicherheit beziehungsweise auf Anwendungen und Produkte beobachten. Weitere Themen des Regulierungsdiskurses umfassen ethische Fragen, allgemeine Themen des Risikomanagements, die Frage nach der Implementierung, nach internationalen Abstimmungen, Fragen der Standardisierung und nach einheitlichen Definitionen.

Die vorliegende Untersuchung hat gezeigt, dass sich das Motiv für Regierungsakteure, eine freiwillige Regulierungsmaßnahme zu ergreifen, teilweise aus der fehlenden Zuständigkeit ergibt. Darunter fällt beispielsweise die Empfehlung eines Verhaltenskodexes für die verantwortungsvolle Nanotechnologieforschung der Europäischen Kommission. Da die Kommission für die Regulierung ethischer Fragen in der Forschung kein unionsrechtliches Mandat besitzt, erfolgt die Regulierung dieses Bereiches über rechtlich nicht verbindliche Akte, wie Kommissionsempfehlungen (vgl. Eisenberger, et al. 2010). Wie die in dieser Studie geführten Interviews gezeigt haben, liegt in anderen Fällen die Idee darin, eine Regulierungsmaßnahme in einer frühen Phase der technologischen Entwicklung $\mathrm{zu}$ ergreifen, in welcher der zu regulierende Gegenstand noch nicht abschließend bestimmt ist. Dies lässt sich insbesondere bei den freiwilligen Datenmeldeverfahren in den Vereinigten Staaten und in Großbritannien beobachten. Ergreifen Nichtregierungsakteure wie Hersteller, Branchenverbände, Denkfabriken und Umweltorganisationen selbstregulatorische 
Maßnahmen, so geschieht dies hauptsächlich im Kontext der Wahrnehmung einer fehlenden gesetzlichen Regulierung.

Durch die Problematisierung der Unkalkulierbarkeit der Risiken bzw. des Nichtwissens bezüglich potenzieller Nebenfolgen der Nanotechnologie durch unterschiedliche Akteure wie z.B. der Swiss Re (Swiss Re 2004) oder der Royal Society (RS\&RAE 2004), wird von einem Lernprozess in Wirtschaft und Politik und bei weiteren gesellschaftlichen Akteure gesprochen (vgl. Lösch 2012; Rip \& van Amerom 2009). Dieser zielt darauf ab, in Eigeninitiative neue Formen der Regulierung im Sinne einer ,verantwortungsvollen governance' zu etablieren, die Lösch $(2012,175)$ als ,selbstregulatorisches Nichtwissensmanagement' bezeichnet. Verschiedentlich wird dabei das Fazit gezogen, dass die ,Governance' der Nanotechnologie eine neue Art der Regulierung darstellt, die den ,verantwortlichen' Umgang zu ihrem Leitmotiv erklärt (Kaiser 2015). Ihr Hauptmerkmal wird in der Vorwegnahme potenzieller Konflikte und der ,präventiven Entleerung' derselben gesehen, um das wissenschaftspolitische Konstrukt der Nanotechnologie zu stabilisieren (vgl. Åm 2011; Kaiser 2015; Wullweber 2009). Demgegenüber wird in dieser Studie davon ausgegangen, dass die im Nanotechnologiediskurs beobachtbare verstärkt netzwerkförmige Regulierung weniger zu einer Entleerung von Konflikten, als vielmehr zu einer gesellschaftlichen Distribution der regulatorischen Verantwortung und somit zu einer Entleerung der institutionellen Zuständigkeit führt.

In diesem Kapitel das doppelte Fazit gezogen, dass einerseits eine Korrelation zwischen dem Maß an Nichtwissen und dem Formalisierungsgrad der Regulierung besteht und andererseits die Regulierung zunehmend gesellschaftlich distribuiert wird. Je unbestimmter also der Regulierungsgegenstand ist, desto stärker werden freiwillige Maßnahmen, wie Verhaltenscodizes, allgemein formulierte Richtlinien und freiwillige Verfahren angewendet. Durch die Beteiligung unterschiedlichster auch traditionellerweise nicht an der Gesetzgebung beteiligter Anspruchsgruppen an der Regulierung der Nanotechnologie und durch die Unverbindlichkeit von selbstregulatorischen Maßnahmen, wird die Regulierungsverantwortung vermehrt an die Gesamtgesellschaft abgetreten. Damit wird der Gültigkeitsbereich von Regulierung zunehmend unklar. Wie diese Analyse weiter zeigt, werden solche Regulierungsmaßnahmen sowohl von Regierungs- wie auch von Nichtregierungsakteuren und oftmals auch in unüblichen Kooperationen ergriffen, wie diejenige des multinationalen Chemikalienherstellers DuPont und der Umweltorganisation Environmental Defense Fund. Ge- 
setzliche Maßnahmen werden anwendungs- oder produktorientiert getroffen, wie beispielsweise im Fall der Regulierung von Nanosilber unter der Pestizidgesetzgebung durch die US-amerikanische Umweltbehörde (Kapitel 3.3.2) oder in demjenigen der Regulierung von Nanomaterialien in Kosmetika und in Lebensmitteln auf Unionsebene.

Die Ergebnisse dieser Untersuchung zeigen, dass freiwillige und informelle Regulierungsmaßnahmen häufig scheitern. Durch die doppelte Unsicherheit, sowohl gegenüber dem zu regulierenden Gegenstand, als auch hinsichtlich der Verbindlichkeit der Maßnahme, haben freiwillige Ansätze im Nanotechnologiediskurs kaum ihre Ziele erreicht. Sie haben weder zu einer höheren Rechtssicherheit beigetragen noch sind sie in der Lage, als Ersatz einer gesetzlichen Regulierung Gesellschaft und Umwelt nachweislich vor nachteiligen Implikationen der Nanotechnologie zu schützen. Faktisch bleiben Nanomaterialien trotz des Vorhandenseins freiwilliger Ansätze unreguliert. Die im neoliberalen Kontext häufig vertretene Haltung, dass Gesetze Innovationen hemmen, wird in dieser Studie widerlegt. Die im Rahmen dieser Studie erhobenen Interviewdaten deuten vielmehr darauf hin, dass eine gesetzliche Regulierung den rechtlichen Spielraum und die Haftungssituation für die Industrie klärt und dass sich die dadurch geschaffene Rechtssicherheit förderlich auf die Investoren, die öffentliche Wahrnehmung und somit auch auf die Ermöglichung von Innovationen auswirkt. Eine klare gesetzliche Regulierung wirde gerade in Bereichen hohen Nichtwissens als innovationsfördernd wahrgenommen.

Die nachfolgenden Kapitel beleuchten die unterschiedlichen Regulierungsansätze fallspezifisch in den untersuchten Ländern und auf der Unionsebene. Dabei steht die Frage im Zentrum, wie diese Maßnahmen den Diskurs prägen und was sie über den gesellschaftlichen Umgang mit Nichtwissen aussagen. Dabei wird der Fokus auf zwei Schwerpunkte gelegt: Einerseits wird die gesetzliche bzw. die behördliche Behandlung der Nanotechnologie im Kontext des Umwelt- und hier insbesondere des Chemikalienrechts nachgezeichnet. Andererseits werden Beispiele von durch regierungsexterne Akteure ergriffene freiwillige Regulierungsansätze diskutiert. Wie in den vorangegangenen Kapiteln werden die verwendeten Praktiken, die behandelten Themen und die involvierten Akteure länderspezifisch untersucht und die in den Interviews erwähnten Ansätze fallweise und beispielhaft dargestellt. Dies geschieht ohne Anspruch auf eine vollständige, flächendeckende Darlegung. In einigen Fällen äußern sich die Interviewpartner ausführlicher, in anderen kaum. Diese ersteren Fälle werden auch hier ausführlicher dargelegt. Dies ermöglicht einen exempla- 
rischen Einblick in die relevanten Themen, Praktiken und Akteurskonstellationen.

\subsection{Vorsorge versus Vermeidung: Zwei Regulierungskulturen}

Eine im Regulierungsdiskurs zentral verhandelte Thematik betrifft die Frage nach der Zuständigkeit der aktuellen Umweltgesetzgebung für die Nanotechnologie. Diese Frage wird sowohl in den Vereinigten Staaten als auch in Europa verhandelt. Wie die Analyse des Abschätzungsdiskurses zeigt, betreffen Fragen des nanotechnologiespezifischen Nichtwissens insbesondere die Umweltbehörden. Im Regulierungsdiskurs akzentuiert sich diese Beobachtung. Aus diesem Grund wird hier der Fokus auf die Diskurse um das Umweltrecht und hier insbesondere auf die Chemikalienregulierung beziehungsweise das Stoffrecht gelegt. Weitere Bereiche des sektoralen Produktrechts wie Lebensmittel und Kosmetika werden hier lediglich fallweise beleuchtet.

Im Umweltrecht wird von einem signifikanten Unterschied zwischen der europäischen und der US-amerikanischen Regulierungkultur gesprochen. Während der europäische Ansatz als ,vorsorgeorientiert' oder risikobasiert bezeichnet wird, wird die US-amerikanische Haltung als ,vermeidungsorientiert' oder wissenschaftsbasiert bezeichnet. Durch die zentrale Etablierung des Vorsorgeprinzips gilt die derzeitige Umweltpolitik der Europäischen Union international als führend. ${ }^{84}$ Die beiden Regulierungskulturen werden verschiedentlich weniger als eine Frage grundsätzlicher regulierungskultureller Unterschiede, sondern als eine im historischen Kontext zeitlich divergierende Entwicklung gesehen. Vogel (2003) beschreibt die Etablierung von Regulierung als zirkulären Trend, der den engen Zusammenhang zwischen öffentlicher Risikowahrnehmung und der Verschärfung der Regulierungspraxis abbildet. Sobald eine strenge neue Regulierungspraxis als funktionierend wahrgenommen wird, reduziert sich der Druck nach noch strengerer Regulierung. In diesem Moment steigt der Einfluss der Industrie, die mit dem Kostenargument Gesetzgeber zu einem Regulierungsstillstand überzeugen, wie dies in den Vereinigten Staaten

84 Die umweltregulatorische Vorreiterrolle Europas wird mit der spezifischen partizipatorisch und kooperativ ausgerichteten politischen Kultur der Europäischen Kommission begründet, in welcher Mitgliedsstaaten über ein umfangreiches Mitspracherecht verfügen und (vgl. Vogel 2003, 191). 
seit den 1990er Jarhen der Fall ist (vgl. Vogel 2003, 198). Der zwischen den 1960er und den 1990er Jahren entwickelte Regulierungsstandard der US-amerikanischen Umwelt- und Konsumentenschutzpolitik wird international als führend, insbesondere auch im Vergleich zu Europa sowohl auf Staaten wie auch auf Unionsebene als innovativer, risikoaverser und robuster beschrieben (vgl. Vogel 2003). In Europa setzt die Umweltpolitik nach 1970 ein und die Risiko-, Gesundheits- und Umweltregulierung wird bis 1990 so vorangetrieben, so dass beide Länder sowohl in Ziel, Ausmass des Schutzes als auch der Vorsorgeorientierung einen ungefähren Gleichsteht erreicht haben (vgl. Christoforou 2004). Dabei vertritt Vogel beispielsweise die Ansicht, dass sich dieses Bild seit Mitte der 1990er Jahren ins Gegenteil gedreht hat und Europa sowohl auf nationaler wie auch auf Unionsebene eine Reihe signifikanter regulatorischer Standarts setzt, die innovativer, risikoaverser und robuster als diejenigen der Vereinigten Staaten sind (vgl. Vogel 2003). Dabei sprechen verschiedene Autoren von Phasen von sich gegenseitig ablösender Vorherrschaft. Im Kontext einer einsetzenden gesellschaftlichen Sensibilisierung für Umweltfragen haben die Vereinigten Staaten bereits in den 1960er Jahren eine wirkungsvolle, vorsorgeorientierte und international führende Umweltregulierung etabliert (vgl. Vogel 2003). Der frühe Start der US-amerikanischen Umweltpolitik wird mit einem damals in der amerikanischen Bevölkerung eingesetzten Umweltbewusstsein begründet. Dieses gilt als durch Publikationen genährt, wie diejenigen über die fatalen Folgen von Pestiziden (Carson 1962), das Ende der Ressourcen, (Meadows, et al. 1972), großtechnische Katastrophen wie das Nuklearunglück in Three Mile Island (Douglas \& Wildavsky 1982) sowie von den im Zusammenhang mit der Raumfahrt entstandenen Bildern, die einen fragilen blauen Planeten Erde in der Endlosigkeit des Weltalls zeigen (Jasanoff 2004). In den 1980er Jahren sind Umweltfragen in den Vereinigten Staaten nicht mehr derart prominent im Fokus der öffentlichen Wahrnehmung gestanden und durch als drängender wahrgenommene Probleme, wie beispielsweise Arbeitslosigkeit und Immigration überlagert worden. Damit ist die Umweltrgulierung zu einem zentralen Bereich geworden, der im Rahmen der neoliberalen Politik der republikanischen Administration unter Präsident Ronald Reagan systematisch abgebaut und geschwächt worden ist. Auch in den nachfolgenden demokratischen Administrationen unter den Präsidenten Carter und Clinton hat die Umweltregulierung keine Stärkung erfahren und das zentrale Konzept der Vermeidensorientierung hat sich weiter etabliert (ibid.). 
Das Einsetzen eines vergleichbaren Umweltbewusstseins in der Zivilbevölkerung lässt sich in Europa erst in den späten 1970er und in den 1980er Jahren im Kontext großtechnischer Störfälle wie dem Reaktorunglück von Tschernobyl und den Chemiekatastrophen von Seveso, Bhopal und Schweizerhalle nachweisen (vgl. Beck 1986; Vogel 2003, 193). Im Jahr 1992 erhält das im Umweltrecht der Europäischen Union zentral verankerte Vorsorgeprinzip durch seine Erwähnung im Vertrag von Maastricht konstitutionellen Status. Die Etablierung des Vorsorgeprinzips auf Unionsebene wird dabei als eine ,Strategie der Bearbeitung von Nichtwissen' beschrieben (Böschen \& Wehling 2012). So sehen die Autoren das epistemische Problem des Nichtwissens beispielsweise im europäischen Chemikalienrecht REACH insofern behandelt, als dass eine Perspektive institutionalisiert wird, die neben toxikologisch nachweisbaren Parametern auch sogenannt weiche Indikatoren für das mögliche Gefährdungspotenzial von Chemikalien berücksichtigt (ibid.). Als Ergänzung dazu wird durch die Einsetzung einer zuständigen Behörde - in diesem Fall die Europäische Chemikalienagentur (ECHA) - ermöglicht, risikorelevantes Wissen aufzubauen. Damit wird der Bereich des unerkannten Nichtwissens strukturiert und wissenschaftlich und politisch anschlussfähig gemacht (vgl. Böschen \& Wehling 2012).

Nach seiner Erwähnung im Verlag von Maastricht wird das Vorsorgeprinzip zum Leitmotiv der Europäischen Umweltpolitik. ${ }^{85}$ Dieses ermöglicht die Regulierung auch bei Verdachtsmomenten und Unsicherheiten, die sich mit traditionellen wissenschaftlichen Methoden wie Kausalität kaum nachweisen lassen (vgl. Christoforou 2004; Raffensperger \& Tickner 1999,23). Das Vorsorgeprinzip wird in einer Mitteilung der Europäischen Kommission ausgehend von drei Grundsätzen beschrieben; 1) Anwendung auf Basis einer möglichst umfassenden wissenschaftlichen Be-

85 Der Ursprung der europäischen Vorsorgeorientierung wird im Deutschland der 1970er Jahre im Kontext eines zunehmenden Bewusstseins der ökonomischen und gesundheitlichen Konsequenzen von Umweltproblemen und in einem wachsenden Unbehagen gegenüber der wissenschaftlichen und politischen Entscheidungsfindung in Risikosituationen verortet. Im Jahr 1971 wird sie im ersten Umweltprogramm der Bundesregierung als zentrales umweltpolitisches Handlungsprinzip festgelegt und findet in den 1980er Jahren zunehmend Eingang in die internationale Umweltpolitik, wie in die von der UN-Generalversammlung beschlossene Weltcharta für die Natur (Erdcharta) von 1982 und in die Rio-Deklaration zu Umwelt und Entwicklung (Agenda 21) von 1992 (vgl. Selin 2007b; Tickner 2003; Vogel 2003, 179). 
wertung unter Berücksichtigung der wissenschaftlichen Unsicherheit, 2) Bewertung der Risiken und potenziellen Folgen einer Untätigkeit bei Entscheidungen für oder gegen bestimmte Tätigkeiten sowie 3) Einbindung der Betroffenen in die Entscheidungsfindung (COM 2000). Zudem soll das Vorsorgeprinzip der Politik ermöglichen, die menschliche Gesundheit und die Umwelt auch angesichts einer unsicheren Risiko- und wissenschaftlichen Faktenlage zu schützen. Eine in den Vereinigten Staaten verbreitete Definition sieht das Vorsorgeprinzip als durch vier zentrale Komponenten definiert; 1) Präventive Handlungen im Fall von Unsicherheit ergreifen, 2) Beweislastumkehr zu den Herstellern oder Befürwortern potenziell schädlicher Handlungen, 3) Alternativen zu potenziell schädlichen Aktivitäten entwickeln und 4) erhöhte öffentliche Einbindung in wissenschaftliche und technische Entscheidungsfindung (Ashford, et al. 1998). ${ }^{86}$

Damit trägt das Vorsorgeprinzip zu einer Bedeutungsverschiebung bei, die weg von der wissenschaftlichen Expertise hin zu wissenschaftsexternen Abschätzungen als Grundlage für regulatorische Entscheidungen im politischen Diskurs führt (vgl. Vogel 2003). Das Vorsorgeprinzip wird insbesondere bei der Regulierung von Technologien angewendet, denen die Öffentlichkeit kritisch gegenübersteht, wie z.B. der Gentechnik und toxischen Chemikalien. ${ }^{87}$ In der Folge wird das Umweltrecht auf Unionsebene stark ausgebaut. Sowohl auf Staaten- wie auch auf Unionsebene werden hohe Standards etabliert. Dabei wird von einer, Risikobasierung' des Europäischen Umweltrechtes gesprochen (vgl. z.B. Tickner 2003; Vogel 2003).

Demgegenüber basiert die dem US-amerikanischen Umweltrecht derzeit zugrunde liegende Vermeidungsorientierung auf der politischen Haltung, dass regulatorische Maßnahmen nur dann ergriffen werden können,

86 Ursprünglich hat die Beweislast im Europäischen Chemikalienrecht bei den Behörden gelegen, erst bei der Etablierung von REACH wird die Beweislastumkehr hin zu den Herstellenden etabliert (vgl. Selin 2007b, 76).

87 Vogel (2003) stellt seine Etablierung in der Umweltpolitik in den Kontext eines Vertrauensverlustes der Bevölkerung in den Nutzen wissenschaftlich-technologischer Entwicklung nach einer Reihe großtechnischer Unfälle, wie Tschernobyl und Schweizerhalle in den späten 1980er Jahren, Lebensmittelskandalen wie BSE in den 1990ern sowie einer dem wissenschaftlich, technologischen Fortschritt zunehmend kritisch eingestellten Bevölkerung. Das Fehlen einer direkten kausalen Verbindung zwischen dem vermuteten Verursacher und dem Schaden wird in unterschiedlichen nationalen Umweltgesetzen und internationalen Umweltabkommen zitiert (vgl. Christoforou 2004, 21). 
wenn wissenschaftlich gesicherte Nachweise für eine Schädigung vorliegen. ${ }^{88}$ Dabei wird von einer, Wissenschaftsbasierung' des Umweltrechts bzw. einem der US-amerikanischen Regulierungskultur inhärenten Ansatz gesprochen, nur dann regulatorisch aktiv zu werden, wenn Schäden wissenschaftlich zweifelsfrei erwiesen sind (vgl. Brickman, et al. 1985; Vogel 1986). In ihrer vergleichenden Analyse der Gentechnikregulierung bezeichnet Jasanoff (1995) den US-amerikanischen Ansatz als ,produktorientiert'. Den Begriff der Produktorientierung versteht sie als auf dem Substanzäquivalenzprinzip basierend, was bedeutet, dass Produkte unabhängig ihres Herstellungsprozesses regulatorisch gleich behandelt werden. Gentechnisch veränderte Pflanzen werden in den Vereinigten Staaten also — im Unterschied zur ,prozessorientierten' Europäischen Gesetzgebung, die in der regulatorischen Behandlung vom Herstellungsprozess ausgeht — gesetzlich wie herkömmlich gezüchtete Pflanzen behandelt.

Zudem sieht die US-amerikanische Regulierungskultur für die Behörden eine doppelte Last vor: Diese müssen sowohl regulatorische Aufgaben übernehmen als auch den wissenschaftlichen Nachweis für deren Notwendigkeit erbringen. Dies erschwert den Regulierungsprozess insbesondere im Bereich von neuen und komplexen Technologien. Zudem trägt dies zu einer zunehmenden Kluft zwischen den sich rasch verändernden Technologien und ihrer Regulierung bei, die mit diesen Entwicklungen kaum Schritt halten kann und somit laufend veraltet (vgl. Marchant, et al. 2007, 203). In diesem regulierungskulturellen Spannungsfeld steht in den Vereinigten Staaten keine mit der Europäischen Vorsorgeorientierung vergleichbare politische Möglichkeit zur Verfügung, bei einer Gefährdungsvermutung regulatorische Maßnahmen zu ergreifen. ${ }^{89}$

Das europäische Umweltrecht ist durch seine Vorsorgeorientierung und Risikobasierung verursacherorientiert angelegt. Mittels Anwendung der ,Beweislastumkehr' müssen die Produzenten die Sicherheit ihrer Stoffe nachweisen, um für diese eine Marktzulassung zu erhalten. Demgegen-

88 Zudem wird die US-amerikanische Regulierungskultur als hoch legalistisch und kompetenztrennend beschrieben. Dadurch erscheint der Regulierungsapparat als träge und einmal eingesetzte Statute, die zwar als überholt gelten, wie z.B. der Clean Water Act und TSCA, werden kaum innert nützlicher Frist überarbeitet (vgl. Marchant, et al. 2007, 201).

89 Zu kulturellen Prägungen der US-amerikanischen Umwelt-, Gentechnik- und Chemikalienregulierung bzw. zu Regulierungsstilen (vgl. z.B. Brickman, et al. 1985; Gottweis 1998; Jasanoff 1995; Vogel 1986; Wright 1994). 
über obliegt beim US-amerikanischen Umweltrecht der Gefährdungsnachweis bei den Behörden (P.L. 91-190 1969). Die Ablösung der Vereinigten Staaten in ihrer Vorreiterrolle der Umweltregulierung durch die Europäische Union wird auf Faktoren, wie die politische Institutionalisierung des Umweltgedankens in grünen Parteien, die in den 1990er Jahren auf Unionsebene erfolgt, zurückgeführt. Ein weiterer Faktor betrifft die Zentralisierung der Regulierung und der hohe Einfluss von Verantwortlichen aus den sogenannt ,grünen' Mitgliedsstaaten in der Kommission (vgl. Christoforou 2004). ${ }^{90}$ Diese seit Mitte der 1990er Jahren zunehmend divergierende umweltpolitische Haltung zwischen den Vereinigten Staaten und Europa prägt auch den Regulierungsdiskurs der Nanotechnologie.

\subsubsection{Nanomaterialien zwischen Vorsorge und Vermeidung}

Vergleichbar zum Abschätzungsdiskurs (Kapitel 2) lässt sich auch im Regulierungsdiskurs ein frühzeitig einsetzender verstärkter Fokus auf Nanomaterialien feststellen. Dies führt dazu, dass insbesondere das Stoffrecht hier eine zentrale Rolle spielt. Auf der Ebene der Europäischen Union ist dies die am 1. Juni 2007 in Kraft gesetzte Chemikalienverordnung REACH (EC 1907/2006 2007). Sie gilt als einer der umfangreichsten umweltregulatorischen Rechtsakte weltweit und hat das bisherige Chemikalienrecht der Union grundlegend harmonisiert und vereinfacht (vgl. Selin 2007b, 64). Zudem steht REACH für ein zentral dem Vorsorgeprinzip unterliegendes Gesetz. Denn in Artikel 1 wird explizit auf die Anwendung des Vorsorgeprinzips auf sämtliche Bestimmungen der Verordnung hingewiesen (ibid.). Bislang sind in REACH keine für Nanomaterialien spezifischen Regeln verankert; Nanomaterialien werden aber auch nicht explizit ausgeschlossen.

90 In den Vereinigten Staaten werden sowohl der vielzitierte neoliberalistisch geprägte Regulierungsabbau unter der Administration der Präsidenten Reagan und Bush verantwortlich gemacht, als auch die umweltpolitische Stagnation in der Clinton Ära sowie die fehlende politische Institutionalisierung von Umweltschutz durch die Parteien. Ebenso wird die Verschiebung der öffentlichen Aufmerksamkeit weg von Umweltfragen hin zu anderen Bereichen öffentlicher Besorgnis wie Arbeitslosigkeit, Sozialversicherungen und die Immobiliernkrise. Dies wird auch verantwortlich gemacht für die Verschiebung des Aufmerksamkeitsfokus der Behörden weg von ihrem Nutzen, stärker auf die Kosten und den Aufwand der Umweltregulierung (vgl. Vogel 2003). 
In den Vereinigten Staaten wird die regulatorische Behandlung von Nanomaterialien hauptsächlich unter dem Kontrollgesetz für Toxische Substanzen (Toxic Substance Control Act, TSCA) diskutiert (P.L. 94-469 1976). TSCA ist im Jahr 1976 entwickelt worden, um eine Regulierungslücke im Bereich toxischer Chemikalien zu füllen, die durch den damals medienspezifisch-orientierten Gesetzgebungsansatz, der die Medien Luft, Wasser und Boden, nicht aber die Stoffe reguliert hat, nicht abgedeckt gewesen ist. Ebenfalls hat die Etablierung von TSCA darauf abgezielt, der EPA die übergreifende Kompetenz zur Chemikalienregulierung zu erteilen (vgl. Marchant, et al. 2007, 193).

Wie für das Umweltrecht als solches beschrieben, sind auch die beiden Statute REACH und TSCA durch die transatlantisch unterschiedlichen Regulierungskulturen geprägt. Die Beweislast der Chemikaliensicherheit liegt in den Vereinigten Staaten ebenso bei den Behörden, wie sie in der Europäischen Union den Herstellern übertragen wird. ${ }^{91}$ Dies führt zu einem zentralen Hindernis für die US-amerikanische Umweltbehörde, Chemikalien zu regulieren, da sie die wissenschaftlichen Nachweise für ein im Statut als ,unverhältnismäßig' bezeichnetes Risiko vorlegen muss, bevor sie regulatorisch aktiv werden kann (vgl. Marchant, et al. 2007, 193). Zudem liegt die grundsätzliche Logik der Chemikalienregulierung TSCA auf der Auffassung, dass sämtliche Chemikalien als sicher gelten, bevor ihre Gefährlichkeit wissenschaftlich nachgewiesen worden ist. Angesichts der komplexen Stoffgemische ist ein solcher Nachweis meist kostenintensiv und kaum zu erbringen (ibid.).

In TSCA unterscheidet der US-amerikanische Gesetzgeber zwischen existierenden Chemikalien (Altstoffen), die nicht unter die gesetzliche Regulierung fallen und neuen Chemikalien (Neustoffen), also solchen mit einer neuen chemischen Identität und einem jährlichen Produktionsvolumen von über 11 Tonnen. Neustoffe müssen vor ihrer Vermarktung bei der Umweltbehörde registriert werden. Die von der Umweltbehörde zu erbringende Sicherheitsprüfung der Chemikalien unter TSCA erfolgt dabei strukturbasiert. Wie ein Interviewpartner erläutert, entscheidet die EPA aufgrund der molekularen Struktur der Chemikalien und damit verknüpf-

91 Das Europäische Umweltrecht wird verursacherorientiert angelegt und basiert auf der ,Beweislastumkehr'. Das bedeutet, dass der Sicherheitsnachweis von Stoffen den Herstellern obliegt. Demgegenüber liegt beim vermeidungsorientiert angelegten US-amerikanischen Umweltrecht der Gefährlichkeitsnachweis bei den Behörden (P.L. 94-469 1976). 
ten Erfahrungswerten bezüglich bestimmter Stoffeigenschaften darüber, ob ein Hersteller zusätzliche Informationen abgeben muss oder nicht:

„Die Kategorien, mit denen wir arbeiten, sind Analogieschlüsse zu anderen Chemikalien. Wir schließen aufgrund ihrer Strukturen und Attribute auf bestimmte Reaktionsweisen und wir arbeiten ausgehend von der Annahme, dass die neuen Chemikalien so gefährlich sind wie das problematischste Exemplar der jeweiligen Kategorie." (Nanotechnologieverantwortlicher, EPA, 22. Januar 2008, Übersetzung der Autorin).

Im Gegensatz dazu gilt die REACH Verordnung nicht nur für Neustoffe, sondern auch für sich bereits auf dem Markt befindende Altstoffe, die über einem jährlichen Produktionsvolumen von einer Tonne hergestellt werden (EC 1907/2006 2007). Diese müssen ebenso unter REACH registriert und auf ihre Ungefährlichkeit hin überprüft werden wie Substanzen, deren Marktzulassung zum ersten Mal beantragt wird. Zudem bietet REACH den Herstellern starke Anreize zur Substitution gefährlicher Substanzen (vgl. Selin 2007b, 76). Neben der Beweislast für die Ungefährlichkeit eines Stoffes müssen die Hersteller Testdaten erheben und diese hinsichtlich ihrer Unbedenklichkeit interpretieren und klassieren. Auch die Einführung abgestimmter Risikomanagementsysteme liegt gemäß REACH Verordnung bei den Herstellern. REACH und die ihr zugrunde liegende Vorsorgeorientierung wird aus Umwelt-, Gesundheits- und Sicherheitsperspektive als starke Regulierung bewertet. Demgegenüber wird TSCA als schwaches Statut kritisiert (vgl. Selin 2006). Diese Einschätzung findet sich auch in den Interviews. Nach Aussage eines Interviewpartners der Europäischen Kommission gilt REACH insbesondere wegen der Beweislastumkehr zulasten der Hersteller als weltweit führende Stoffrechtsverordnung:

„Die grundlegende Idee von REACH ist, dass individuelle Hersteller oder Importeure einer bestimmten Substanz verantwortlich sind, die Gesundheitsund Sicherheitsstandards zu entwickeln und zu kommunizieren. Wir haben das weltweit führende, und ich darf das sagen, da ich kein anderes Land kenne, das ein nicht einmal zu 10\% mit der Konsequenz von REACH vergleichbares Stoffgesetz besitzt." (Nanotechnologieverantwortlicher, Europäische Kommission, DG Umwelt, 30.10.2008, Übersetzung der Autorin).

Die transatlantischen Unterschiede in der Behandlung von Chemikalien sind den US-amerikanischen Behördenmitgliedern bekannt, sie werden jedoch als politisch konstruiert angesehen. Dazu erläutert ein Interviewpartner der US-amerikanischen Umweltbehörde, dass in technischen Fragen oder in der Implikation kaum Unterschiede bestehen. Er vertritt die Haltung, dass die übergeordneten Ziele trotz der unterschiedlichen Regulie- 
rungsauffassungen letztendlich überall die gleichen sind, nämlich Daten zu generieren, um die Umwelt und die öffentliche Gesundheit vor Chemikalien zu schützen:

\begin{abstract}
„Wir haben selten fachliche Differenzen zwischen den Vereinigten Staaten und Europa. Wir haben politische Differenzen und Differenzen in der Art und Weise, wie wir die Dinge betrachten. Aber grundsätzlich wollen wir alle eine ausreichende Anzahl an Daten erheben, um die Chemikalien zu überprüfen, die sich in der Umwelt befinden und denen Menschen ausgesetzt sind und wir möchten Entscheidungen zum Schutz von Gesundheit und Umwelt treffen. Wir verfangen uns in Begriffen, wie 'Vorsorgeprinzip', 'gefahrenbasiert', 'risikobasiert' und so weiter. Dabei handelt es sich um politische Argumente im Unterschied zu wirklichen Substanzargumenten. Denn letztendlich haben wir beide die gleichen fachlichen Ziele." (Nanotechnologieverantwortlicher, EPA, 22. Januar 2008, Übersetzung der Autorin).
\end{abstract}

Ein Interviewpartner einer Umweltorganisation weist auf den kulturellen Unterschied der beiden Regulierungen bezüglich der gesetzlich festgelegten Fakten und ihrer Implementierung hin. So erläutet er, dass insbesondere US-amerikanische Firmen die Einschätzung vertreten, dass das europäische System robustere Gesetze auf dem Papier hat, diese jedoch kaum in ihrer vollumfänglichen Konsequenz durchsetzt, während demgegenüber die Gesetzestexte in den Vereinigten Staaten schwächer formuliert sind, aber konsequenter umgesetzt werden:

„US-amerikanische Firmen sagen normalerweise, dass Europa auf dem Papier strenge Gesetze hat, aber dass es diese nicht wirklich durchsetzt. Demgegenüber haben wir in den Vereinigten Staaten möglicherweise Gesetze, die nicht ganz so weit gehen, aber sie werden so durchgesetzt, dass wir sie befolgen." (Chemikalienspezialist, Environmental Defense Fund, 6. März 2008, Übersetzung der Autorin).

Persönlich bezweifelt der Interviewpartner die Richtigkeit dieser Einschätzung, dass in den Vereinigten Staaten die Gesetze konsequenter eingehalten werden:

„Irgendwie bezweifle ich, dass wir einen so guten Vollzug haben in diesem Land. Aber ich denke, dass in den Vereinigten Staaten traditionellerweise eine antagonistischere Beziehung zwischen der Regierung und der Industrie besteht als in Europa. Ob dies im einen oder im anderen System zu einer besseren Einhaltung der Gesetze führt, das kann ich nicht beurteilen." (Chemikalienspezialist, Environmental Defense Fund, 6. März 2008, Übersetzung der Autorin). 
Allerdings bewertet er die Beziehung zwischen der Industrie und den Regierungsbehörden in den Vereinigten Staaten als feindseliger als in Europa.

\subsubsection{Innovation versus Regulierung - ein Mythos?}

Zudem zweifeln verschiedene Interviewpartner die vielzitierte und die neoliberale Politik leitende Haltung an, dass eine gesetzliche Regulierung der Industrie schadet - oder anders gesagt, dass sich Regulation und Innovation ausschließen. Vielmehr gehen sie von einer innovationsfördernden Wirkung einer klaren gesetzlichen Regelung aus und vertreten die Haltung, dass sich die Industrie Rechtssicherheit wünscht. Ihrer Ansicht nach klärt eine gesetzliche Regulierung den rechtlichen Spielraum und die Haftungssituation, was sich förderlich auf Innovationen und die öffentliche Wahrnehmung auswirkt. Zudem würden Produkte im unregulierten System wegen Rechtsunsicherheit an Konkurrenzfähigkeit einbüßen. Zugleich wird argumentiert, dass eine fehlende gesetzliche Regulierung in der Bevölkerung Wiederstand hervoruft, was sich ebenfalls nachteilig auf die Branche auswirkt.

Dies deutet darauf hin, dass eine gesetzliche Regulierung gerade in Bereichen, in denen ein hohes Maß an Nichtwissen besteht, als innovationsfördernd wahrgenommen wird. Dazu erwähnt ein Interviewpartner des Wilson-Zentrums, dass auch die Industrie an einer gesetzlichen Regulierung interessiert ist. Am Beispiel der unregulierten US-amerikanischen Biotechnologieindustrie erläutert er, dass ein unregulierter Markt zu Haftungsunsicherheiten führt und ein regulierter Markt dadurch über Wettbewerbsvorteile verfügt. Dies führt dazu, dass zunehmend auch die Industrie die gesetzliche Regulierung der Nanotechnologie einfordert:

„In den vergangenen Jahren ist die Industrie sogar nach Washington gekommen und hat darum gebeten, reguliert zu werden. Ohne Regulierung, fehlen der Biotechnologieindustrie die Investoren. Die Industrievertreter befürchten Haftungsfälle und im regelfreien System von chinesischen Produkten unterboten $\mathrm{zu}$ werden. Auch befürchten sie eine unausgewogene Regulierungslandschaft." (Leitungsmitglied, Woodrow Wilson International Center for Scholars, 4. März 2008, Übersetzung der Autorin).

Die Problematik, dass im unregulierten System Investitionen durch potenzielle Haftungsklagen gebremst werden und Produkte dadurch an Konkurrenzfähigkeit einbüßen, schildert auch eine deutsche Rechtsexpertin und 
Mitverfasserin eines Rechtsgutachtens zur Nanotechnologie. Sie beobachtet, dass industrielle Akteure einer gesetzlichen Regulierung zustimmen, da sie befürchten, dass Produkte im unregulierten System an Konkurrenzfähigkeit einbüßen:

„Aus Industriesicht sollte man auch sehen, dass eine Regulierung zu Rechtssicherheit beiträgt. Die fehlende Regulierung ist ein Zustand, der von der Industrie auch oftmals beklagt wird.“ (Rechtsanwältin, Mitverfasserin des Rechtsgutachtens ReNaTe, 16.9.2008).

Ein ebenfalls in die Verfassung des Rechtsgutachtens Nanotechnologie involvierter Interviewpartner stellt eine Analogie zum Gentechnikdiskurs her. Dabei vertritt er die Ansicht, dass die fehlende gesetzliche Regulierung nicht im Interesse der Industrie sein kann. So hat die Rechtsunsicherheit in der Gentechnik zu einem Akzeptanzverlust in der öffentlichen Wahrnehmung geführt, was sich bei der Nanotechnologie wiederholen kann:

„Die Industrie müsste eigentlich an klaren Spielregeln interessiert sein, um ihre Eigenverantwortung in einem vernünftigen Rahmen entfalten zu können. Denn sonst droht ihnen die Gefahr, dass sie ein Desaster erleiden wie bei der Gentechnik. Wenn die Nanotechnologie nämlich unreguliert ist, dann ist auch unklar, was sie nun wirklich machen müssen, und der Vorwurf, sie haben nicht genug gemacht, ist in der öffentlichen Diskussion schnell erhoben." (Leitungsmitglied, Sonderforschungsgruppe Institutionenanalyse (sofia), Hochschule Darmstadt, 9.9.2008).

Auch im internationalen Diskurs wird die Beobachtung geäußert, dass sich die Industrie eine gesetzliche Regulierung wünscht. So erläutert ein Interviewpartner der OECD-Arbeitsgruppe Nanomaterialien, dass die Firmen über die regulatorische Unsicherheit besorgt sind:

„Die Firmen scheinen besorgt zu sein über die regulatorischen Unsicherheiten, insbesondere bezüglich der EHS-Forschung und der Umwelt. Sie möchten Klarheit haben über die Regulierung. Den Firmen ist nicht klar, welche Regulierung für ihre Produkte in welchem Land gilt und es scheint, dass dies einige Investitionen zurückhält. Darüber besteht Besorgnis sowohl in Europa, in den Vereinigten Staaten als auch in Asien." (OECD Verantwortlicher, Science and Technology Policy, Working Party on Nanotechnology, 9. Dezember 2008, Übersetzung der Autorin).

Derselbe Interviewpartner vertritt die Haltung, dass eine Klärung der rechtlichen Situation vonseiten der nationalen Regierungen notwendig ist:

„Regierungen müssen die Situation klären. Natürlich haben wir Gesetze in Europa. Es gibt den Verhaltenskodex der Europäischen Union. Aber dann kommen Firmen und die Industrie zusammen und schlagen freiwillige Sche- 
men vor. Darin scheint viel Unsicherheit zu gründen. Und es gibt Firmen, die Instrumente zum Testen von Nanomaterialien entwickeln. Diese erhoffen sich auch mehr Klarheit zu bekommen, so dass sie wissen, welche Instrumente für EHS-Analysen benötigt werden." (OECD Verantwortlicher, Science and Technology Policy, Working Party on Nanotechnology, 9. Dezember 2008, Übersetzung der Autorin).

Die unterschiedlichen freiwilligen und selbstregulatorischen Maßnahmen der Industrie und der Regierungen erachtet er für die Herstellung der Rechtssicherheit als nicht besonders hilfreich. Die traditionelle Sichtweise, dass sich Regulation und Innovation ausschließen, scheint sich also im Nanotechnologiediskurs nicht zu bestätigen.

\subsubsection{Verschwimmende Regulierungskulturen durch internationale Kooperation}

Sowohl in der Literatur als auch in den Interviews wird von divergierenden Regulierungskulturen zwischen dem europäischen und dem US-amerikanischen Umweltrecht gesprochen, so dass auch für die Regulierung der Nanotechnologie das zuständige Stoffrecht gilt. Während das US-amerikanische Umweltrecht als vermeidungsorientiert, schadens- oder wissenschaftsbasiert beschrieben wird, gilt das europäische als vorsorgeorientiert oder risikobasiert. Diese unterschiedlichen Ansätze betreffen insbesondere Fragen nach der Beweislast und nach den getesteten Substanzen (Alt- und Neustoffe/Volumen). Dennoch deuten verschiedene im Rahmen dieser Untersuchung erhobene Aspekte auf ein Verschwimmen dieser ausgeprägten regulierungskulturellen Unterschiede im Nanotechnologiediskurs hin.

Einerseits zeigen sich diese regulierungskulturellen Unterschiede hauptsächlich in den Gesetzen und bereits weniger ausgeprägt in der Implementierung. Hierzu wird die Vermutung geäußert, dass die regulatorische Schwäche des US-Systems durch eine konsequente Umsetzung teilweise ausgeglichen wird. Kurz gesagt vertritt diese These die Behauptung, dass die Europäische Union über eine robustere Umwelt- und Chemikalienregulierung, aber über eine schwächere Umsetzung verfügt. Demgegenüber gilt die US-amerikanische Gesetzgebung als schwächer aber deren Umsetzung als konsequenter. Eine weitere Vermutung betrifft den Antagonismus zwischen Industrie und Behörden. Derzufolge gilt die US-amerikanische Industrie als regulierungsaverser als die europäische. Dennoch lässt sich in beiden Regulierungskulturen sowohl in der vermeidungsorientierten der Vereinigten Staaten als auch in der vorsorgeorientierten der Europäischen 
Union der Wunsch der Industrie nach einer robusten gesetzlichen Regulierung und somit nach Rechtssicherheit feststellen.

Andererseits - und dies wird hier als bedeutsamerer Faktor des Verschmelzens der regulierungskulturellen Unterschiede im Nanotechnologiediskurs gesehen - lässt sich ein Bedeutungszuwachs der internationalen Kooperation in Regulierungsfragen beobachten. Da es sich bei der Nanotechnologie um eine global intensiv vorangetriebene Technologie handelt, wird auch das damit verbundene Nichtwissen global wahrgenommen. Damit erscheint es naheliegend, dieses auch auf internationaler Ebene kooperativ anzugehen. Dieses Bestreben nach internationaler Kooperation lässt sich sowohl auf Regierungs- wie auch auf Nichtregierungsebene beobachten und wird hier auch als Delegation der Regulierungsveranwortung bezeichnet. Diese Delegation führt von nationalstaatlichen Regulierungsbehörden weg nicht nur in die Gesamtgesellschaft, sondern auch in supranationale Organisationen wie die Europäische Union aber auch in internationale Regierungsforen wie die Organisation für wirtschaftliche Zusammenarbeit und Entwicklung (OECD), ${ }^{92}$ die Internationale Standard Organisation (ISO) und in private internationale Initiativen, Stiftungen und Kooperationen, wie sie beispielsweise der in Genf ansässige Internationalen Risikoregulierungsrat (IRGC) mit seinem Nanotechnologieschwerpunkt darstellt.

Verschiedene Interviewpartner auf der Länder- und der Unionsebene erwähnen die internationale Kooperation als eine wichtige Strategie im Umgang mit dem mit der Nanotechnologie verbundenen Nichtwissen. Eine solche Zusammenarbeit erfolgt einerseits bilateral zwischen den einzelnen Ämtern. Andererseits werden solche Kooperationen gerade auch im Bereich der Nanotechnologie in internationalen Gremien institutionalisiert. Diese Institutionalisierungen entstehen in unterschiedlichen Bereichen, wie beispielsweise in der Zusammenarbeit über die Definition und die Standardisierung der Nanotechnologie in der Internationalen Standardor-

92 Die OECD wird im Jahr 1961 als Nachfolgeorganisation der nach dem Zweiten Weltkrieg etablierten Organisation für Europäische wirtschaftliche Zusammenarbeit (OEEC) und des Marshallplans zum Wiederaufbau Europas gegründet. Ihr Ziel ist die Förderung des ökonomischen Fortschritts der Mitgliedsstaaten. Die OECD dient als Forum, in welchem die Regierungen der 34 Mitgliedsstaaten meist westliche Industrienationen - ihre Erfahrungen austauschen, für Mitglieder bindende Empfehlungen abgeben und Lösungen für gemeinsame Probleme erarbeiten (vgl. http://www.oecd.org/about/, 23.04.2014). 
ganisation (ISO). Auch im Bereich der Vorsorgeregulierung und der Innovationsförderung werden internationale Kooperationsgremien etabliert, wie die beiden von der OECD eingesetzten Arbeitsgruppen zur Nanotechnologie und zu Nanomaterialien. Ein Industrievertreter beschreibt die internationale Kooperation in solchen Gremien als zentral für die Rechtssicherheit von global agierenden Industrieunternehmen:

„Die OECD und ISO sind komplementär und wichtig. ISO braucht man, um Begrifflichkeiten und Messtechniken festzulegen und OECD, um diese global zu harmonisieren. Deswegen votieren wir dafür, dass die Überprüfung der existierenden Testguidelines bei der OECD stattfindet und nicht im nationalen Kontext. Denn mit dem OECD-Prozess kommen wir automatisch zu global akzeptierten und gemeinsamen Regularien und damit den Welthandel fördernden Techniken.“ (Nanotechnologieverantwortlicher, Evonik/Degussa, HanauWolfgang, 10.9.2008).

Verschiedene Interviewpartner erwähnen die Kooperation auf OECD-Ebene als eine der Strategien im Umgang mit dem mit Nanotechnologie verbundenen Nichtwissen. Dies deutet auf einen auf Regierungsebene identifizierten Kooperations- und internationalen Koordinationsbedarf im Bereich der Nanotechnologie hin. In solchen Fällen gilt die OECD als eine der zentralen Plattformen. ${ }^{93}$ Ihre Rolle liegt in der internationalen Abstimmung über globale Themen, die den Welthandel, die Forschung und die Entwicklung betreffen (vgl. Falkner 2007). Die OECD spielt insbesondere eine wesentliche Rolle als Plattform, auf der verschiedene Wirtschaftsräume, wie beispielsweise die Europäische Union und die Vereinigten Staaten, ihre Risikoregulierungsansätze harmonisieren. ${ }^{94}$ Diese Einschätzung wird in den Interviews geteilt. Dazu stellt ein Mitglied der Europäischen

93 Der OECD kommt zentrale Bedeutung in der internationalen Kooperation zur Harmonisierung der Risikoregulierung zu. Hierin spielt auch die Welthandelsorganisation (WTO) eine wichtige Rolle. Diese ist jedoch nicht Gegenstand dieser Untersuchung. Im Rahmen der OECD werden Vorgaben und Richtlinien verfasst und gelegentlich auch für die Mitglieder rechtlich verbindliche Verträge festgelegt. Die OECD ermöglicht auch Nichtmitgliedsstaaten und regierungsexternen Akteuren die Teilnahme an ihren Aktivitäten. Dabei unterhält die OECD verschiedene Programme, in die sich auch Nichtmitglieder einschreiben können (vgl. http://www.oe cd.org/about/think, 23.04.2014).

94 Im Idealfall trägt dies zu einer hohen Risikovorsorge unter der gleichzeitigen Förderung von wissenschaftlichen und ökonomischen Innovationen bei. Nach Falkner (2007) ergeben sich aus den Divergenzen in der Regulierungskultur, in der öffentlichen Wahrnehmung und in der politischen Dynamik Schwierigkeiten bei der transatlantischen Koordination und Zusammenarbeit. Diese führen zu unterschied- 
Kommission fest, dass die OECD die wichtigste internationale Verhandlungsplattform darstellt:

„In diesem Bereich muss auf der internationalen, auf der globalen Ebene gearbeitet werden und die wichtigste Plattform dafür ist heute die OECD.“ (Nanotechnologieverantwortlicher, Europäische Kommission, DG Unternehmen und Industrie, 3. Dezember 2008, Übersetzung der Autorin).

Die OECD-Arbeit unterscheidet zwischen Nanomaterialien und Nanotechnologie beziehungsweise zwischen Innovations- und Implikationsmanagement. Der Bereich der Nanomaterialien steht für potenzielle Implikationen, insbesondere bezüglich, der Umwelt, der Gesundheit und der Sicherheit (EHS) und für damit einhergehende regulatorische Fragen. Der Bereich der Nanotechnologie fokussiert demgegenüber Forschung und Entwicklung; also hauptsächlich Innovationen und Fragen der Definition. $\mathrm{Zu}$ beiden Bereichen hat die OECD Arbeitsgruppen eingesetzt. Es sind dies die im Jahr 2006 etablierte Arbeitsgruppe Nanomaterialien (WPMN) und die ein Jahr später ins Leben gerufene Arbeitsgruppe Nanotechnologie (WPN).

Die Aufgabe der Arbeitsgruppe Nanomaterialien (WPMN) liegt in der internationalen Harmonisierung von wissenschaftsbasierten Risikoanalysen, Schadens- und Expositionsabschätzungen. Eines der Hauptprojekte dieser Arbeitsgruppe betrifft die Durchführung eines Testprogramms für Nanomaterialien (Sponsorship Programme). Dieses Programm will die Risikoforschung im Bereich der Nanomaterialien international koordinieren und die Sicherheit der 14 meistverwendeten Nanomaterialien testen. Die einzelnen Mitgliedsstaaten teilen sich dabei die Finanzierung und die Durchführung von Sicherheitstests untereinander auf:

„Die aktivste Aufgabe ist derzeit das Testen einer repräsentativen Auswahl an Nanomaterialien. Die Länder haben 14 Nanomaterialien identifiziert, die sie testen möchten. 13 Mitgliedsstaaten haben sich dazu bereiterklärt, das Testen eines Nanomaterials vollständig, teilweise oder mit einem Beitrag zu finanzieren. Wir schätzen, dass das Testen eines dieser Nanomaterialien etwa zwischen 2 und 3 Mio. Euro kosten dürfte. Das ist ein großer Beitrag, den die Länder leisten." (OECD Verantwortlicher, Working Party on Engineered Nanomaterials, 19. Dezember 2008, Übersetzung der Autorin).

Wie verschiedene Mitglieder erläutern, ist die internationale Kooperation in der Arbeitsgruppe Nanomaterialien höchst relevant für den Austausch,

lichen Regulierungsansätzen, wie beispielsweise bei hormonbehandeltem Rindfleisch und gentechnisch veränderten Lebensmitteln (vgl. Falkner, et al. 2010). 
die Abstimmung und die Durchführung von umwelt- und gesundheitsrelevanter Begleitforschung. Weitere Aufgaben betreffen die Überprüfung sämtlicher international verfügbarer OECD-Richtlinien auf ihre Anwendbarkeit auf Nanomaterialien, die Erstellung einer offen zugänglichen Forschungsdatenbank mit Sicherheitsinformationen zu den wichtigsten synthetisch hergestellten Nanomaterialien ${ }^{95}$ und freiwillige Datenmeldesysteme. Wie der OECD-Vertreter erläutert, sind verschiedene Länder an freiwilligen Datenmeldesystemen, wie dem britischen Datenmeldesystem (VRS) interessiert. Im Rahmen dieses Projektes diskutieren die Länder neben freiwilligen auch verbindliche regulatorische Ansätze:

„Wir haben ein Projekt, in dem freiwillige Datenmeldeschemen angeschaut
werden, beispielsweise dasjenige Großbritanniens. Dabei wird geschaut, was
anders ist, was funktioniert, was nicht funktioniert und dann vielleicht auch
die Frage, wie gemeinsame Prinzipien entwickelt werden könnten, um ein
freiwilliges System anzupassen. Es wird sogar über unfreiwillige Maßnahmen
oder Regulierungsmaßnahmen nachgedacht, welche Nanomaterialien betref-
fen. Dabei handelt es sich um einen relativ aktiven Bereich mit intensiven
Diskussionen zwischen den Ländern.“ (OECD Verantwortlicher, Working
Party on Engineered Nanomaterials, 19. Dezember 2008, Übersetzung der
Autorin).

Verschiedene Autoren von Regulierungsanalysen diskutieren die Idee einer koordinierten und weltweit gültigen Nanotechnologieregulierung. Dabei wird als eine der Optionen deren Etablierung auf der OECD-Ebene diskutiert (vgl. Abbott, et al. 2010; Breggin, et al. 2009; Falkner, et al. 2010). ${ }^{96}$ Auch ein Interviewpartner aus einem US-amerikanischen Dialogberatungsinstitut bewertet die OECD-Ebene als den Ort, an welchem derzeit die bedeutendsten Aktivitäten im Regulierungsdiskurs der Nanotechnologie stattfinden. Seiner Ansicht nach könnte dieses Gremium die Basis für eine mögliche Regulierung legen:

„Möglicherweise eine der signifikantesten Aktivitäten, findet auf OECD-Ebene statt. Sie könnte die Grundlage für nützliche Wege liefern, um über Regulierung und den Regulierungsbedarf nachzudenken." (Leitungsmitglied, Meridian Institute, 23. Januar 2008, Übersetzung der Autorin).

95 Siehe http://www.oecd.org/env/ehs/nanosafety/ (16.09.2013).

96 Diesbezüglich werden auch verschiedentlich Aktivitäten des IRGC aufgeführt. In den im Rahmen dieser Studie geführten Interviews zeigt sich jedoch, dass diese kaum Resonanz auf nationaler, internationaler oder supranationaler Ebene erlebten (siehe Kapitel 3.6.2). 
Die anderen, im Rahmen der vorliegenden Studie befragten Interviewpartner, erwähnen diese Option jedoch kaum. Sie vertreten vielemehr die Haltung, dass die internationale Kooperation auf OECD-Ebene dem Austausch von Informationen und der Harmonisierung der Implementierung dient, nicht jedoch der Abstimmung über die Regulierung. Dazu erläutert eine Vertreterin der Europäischen Kommission, dass die Kernkompetenz der OECD-Arbeit in der Harmonisierung der Umsetzung liegt:

„Die OECD kann die Regulierung auf der Implementierungsebene unterstützen, aber sie kann nie die Regulierung als solche betreffen. Die zentrale Priorität liegt bei der Arbeit an der Implementierung. Das Ziel der OECD-Arbeit ist die Gesetzgebungen weltweit in der Harmonisierung ihrer Testrichtlinien zu unterstützen, so dass eine Firma in den Vereinigten Staaten dieselben Testmethoden anwendet wie eine Firma in Japan oder eine Firma in Europa." (Nanotechnologieverantwortliche, Europäische Kommission, DG Umwelt, 13. November 2008, Übersetzung der Autorin).

Ein Interviewpartner der Generaldirektion Unternehmen erläutert, dass vielmehr Implementierungsfragen sowie solche der Terminologie, des Datenaustauschs und des Arbeitsschutzes dazu geeignet sind, auf internationaler Ebene harmonisiert zu werden, als solche der Regulierung:

„Ich denke nicht, dass man auf globaler Ebene regulieren kann. Einigen kann man sich über Terminologien, über Risikoabschätzungsmodelle, über den Datenaustausch. Terminologie, Datenaustausch, Arbeitsschutz, Messen, Testen, Risiko- und Lebenszyklusanalysen, all diese Aspekte können auf der internationalen Ebene geregelt werden." (Nanotechnologieverantwortlicher, Europäische Kommission, DG Unternehmen und Industrie, 3. Dezember 2008, Übersetzung der Autorin).

Ein Vertreter einer US-amerikanischen Umweltorganisation, der als Beobachter in die OECD-Arbeit eingebunden ist, sieht ebenfalls kaum Möglichkeiten, wie die OECD Regulierungsaspekte harmonisieren soll. Vielmehr beschreibt er die Arbeitsgruppe Nanomaterialien als ein Forum, in welchem die Harmonisierung der Implementierung und der Informationsaustausch im Zentrum stehen:

„Die OECD ist hauptsächlich ein Forum zum Austausch von Informationen und zum gemeinsamen Nutzen von Daten, zum Fördern des gegenseitigen Verständnisses und zum Harmonisieren der Ansätze. Sie erlässt, wenn überhaupt, sehr selten Auflagen oder harte Empfehlungen, wie Länder mit bestimmten Chemikalien umgehen sollen und sie macht keine Vorschriften zum Risikomanagement oder zu Risikokontrollen, die von den Mitgliedsstaaten übernommen werden." (Chemikalienspezialist, Environmental Defense Fund, 6. März 2008, Übersetzung der Autorin). 
Ein Vertreter der OECD erläutert, dass trotz der unterschiedlichen Regulierungskulturen der einzelnen Mitgliedsstaaten auf Implementierungsebene eine hohe Übereinstimmung besteht. Die OECD-Arbeit stößt jedoch dort auf ihre Grenzen, wo sie die technischen Belange der Implementierung verlässt und es um gesetzliche Maßnahmen und Regulierungsparadigmen geht. Eine Regulierungsnorm vorzuschlagen, die sämtliche Mitgliedsländer übernehmen müssen, liegt nicht in der Kompetenz der OECD. Deshalb beurteilt er die Frage nach der Umsetzungspraxis als die wichtigere als diejenige nach der Regulierung:

„Wir sind nicht erfolgreich, wenn wir anfangen, Risikomanagementfragen oder dergleichen zu behandeln. Das Regulierungssystem funktioniert anders. Aber viel wichtiger sind die Risikoabschätzung und dabei insbesondere die Datensammlung und der Datenaustausch. Es wäre sehr schwierig, zu sagen, dass die Vereinigten Staaten das REACH-System oder Europa TSCA übernehmen sollte. So funktioniert es nicht. Wichtiger ist die Frage, wie etwas getan werden soll, als auf der Ebene der Regulierung zu denken." (OECD Vertreter, Working Party on Engineered Nanomaterials, 19. Dezember 2008, Übersetzung der Autorin).

Der Fokus der OECD-Arbeitsgruppe Nanotechnologie (Working Party on Nanotechnology, WPN) liegt insbesondere im Bereich der Forschung und Entwicklung, also bei den Innovationen. Wie auch in den nationalen Diskursen, besteht eines der Ziele darin, eine international gültige Definition festzulegen. Zudem umfassen ihre Aufgaben die Erfassung international vergleichbarer und validierter Indikatoren und Statistiken über die Marktentwicklung, die Ausgaben für Forschung und Entwicklung sowie über die Publikationen und Patente für die Politik und die Regierungen. ${ }^{97}$ Wie ein Vertreter der WPN erläutert, liegt die zentrale Aufgabe der Arbeitsgruppe darin, Länder zusammenzubringen und gemeinsam einen Umgang mit nanotechnologischen Herausforderungen zu finden:

„Wir versuchen die Länder zusammenzubringen, um zusammen zu überlegen, was Nanotechnologie ist, was die Möglichkeiten und mögliche Auswirkungen und Risiken sind. Wir diskutieren, tauschen Ergebnisse und Erkenntnisse aus, um das Feld und seine Entwicklung besser zu verstehen." (OECD Vertreter, Science and Technology Policy, Working Party on Nanotechnology, 9. Dezember 2008, Übersetzung der Autorin).

97 Siehe http://www.oecd.org/sti/sci-tech/oecdworkingpartyonnanotechnology.htm (21.9.2013) 
Ein wichtiger Schwerpunkt der WPN-Arbeit liegt auch im Austausch über nationale Regulierungsansätze. Dabei stehen nach Aussage des OECDVertreters insbesondere auch die Schwellen- und asiatische Länder wie China, Russland und deren regulatorische Ansätze im Zentrum des Interesses:

„Es wäre interessant zu erfahren, wie sich die Länder in einigen Vorschriften unterscheiden; wie beispielsweise der US-amerikanische Ansatz, der Ansatz der Europäischen Union aussieht und um zu sehen, welcher Regulierungsansatz in Asien verfolgt wird: $\mathrm{Ob}$ dieser näher bei Europa steht oder bei den Vereinigten Staaten oder ob Asien ein eigenes System entwickelt hat. Und all das hat mit Harmonisierung zu tun; das ist sehr wichtig." (OECD Vertreter, Science and Technology Policy, Working Party on Nanotechnology, 9. Dezember 2008, Übersetzung der Autorin).

Da in Europa bereits vielfältige Institutionen aktiv sind, liegt nach Ansicht des Interviewpartners die Herausforderung darin, Asien und weitere Schwellenländer in die WPN-Arbeit zu integrieren. Dabei stellt für ihn die globale Kooperation eine zentrale Aufgabe dar:

„In Europa gibt es eine Reihe an Institutionen. Es besteht bereits eine Tradition zur Zusammenarbeit in dem Bereich und die Vereinigten Staaten sind involviert. Die Herausforderung liegt darin, Asien zu integrieren. Auch Schwellenländer, die schnell ziemlich wichtig werden, wie China, Russland, Indien und Korea." (OECD Vertreter, Science and Technology Policy, Working Party on Nanotechnology, 9. Dezember 2008, Übersetzung der Autorin).

Auch hinsichtlich eines möglichen Schadensfalls sieht er es als wichtig an, dass ein globales Netzwerk existiert, das die Koordination auf der internationalen Ebene ermöglicht:

„Wenn unerwartete Probleme auftreten, die eine enge Zusammenarbeit erfordern, haben wir zumindest ein Forum, in welchem die Länder in diesen Fragen zusammenarbeiten können." (OECD Vertreter, Science and Technology Policy, Working Party on Nanotechnology, 9. Dezember 2008, Übersetzung der Autorin).

Neben der Kooperation auf der OECD-Ebene, an welcher insbesondere die Regierungen, aber auch Firmen und Umweltorganisationen teilnehmen, lassen sich auch von den etablierten internationalen Organisationen losgelöste internationale Kooperationen beobachten. Eine davon umfasst die nanotechnologiespezifischen Aktivitäten des in Genf (Schweiz) ansässigen Internationalen Risikoregulierungsrats (IRGC), der sich der Entwicklung eines übergreifend wirksamen internationalen Risikosteuerungsschemas verschrieben hat. Mittels Kooperationen mit international renom- 
mierten Experten wie einem der federführenden Köpfe der NNI, Mihail Roco, dem deutschen Risikosoziologen Ortwin Renn sowie dem international tätigen Rückversicherer und Verfasser einer vielbeachteten Abschätzungsstudie zu Nanomaterialien; der Swiss Re (siehe Kapitel 2), hat diese internationale Organisation verschiedene Risikoanalyse-Berichte zur Nanotechnologie verfasst (IRGC 2007; IRGC 2009).

Zudem hat der IRGC einen selbstregulatorischen Lenkungsansatz entwickelt. Dieser in einem Weißpapier publizierte Ansatz nimmt die Form eines konzeptuellen Schemas zur Analyse der Nanotechnologie und fokussiert auf internationale regulatorische und wissenschaftspolitische Aspekte (IRGC 2006). Ziel dieses Schemas ist es gemäß IRGC, Entscheidungsträgern einen ,systematischen' und ,einheitlichen' Ansatz der Risikoanalyse und des Risikomanagements zur Verfügung zu stellen (ibid. S. 12). Darin sollen auch übergreifende Aspekte und transdisziplinäre Fragen behandelt werden, die nicht von einzelnen Anspruchsgruppen alleine bearbeitet werden können. In der im Jahr 2008 im Auftrag des IRGC erstellten Studie zu Nanotechnologie in Lebensmitteln und bei Kosmetika geben die Autoren einen Überblick über die toxikologischen Erkenntnisse und die ergriffenen regulatorischen Maßnahmen. Sie kommen zum Schluss, dass insbesondere die freiwilligen Verhaltensnormen nur dann effektiv sind, wenn sie über die Meinungspflege hinaus, auf echte Produktsicherheit abzielen (Grobe, et al. 2008). ${ }^{98}$ Ein zentral in die Entwicklung der NNI und in die Aktivitäten des IRGC involvierter Interviewpartner erläutert, dass der IRGC die Haltung vertritt, dass die existierende Regulierung zwar die Nanotechnologie erster Generation abdeckt, dass aber für die nächste Generation nanomaterialien-basierter Produkte mit komplexeren interaktiven Systemen eine Regulierungslücke besteht und neue Regulierungsansätze notwendig werden:

„Bislang sind alle Länder zum Schluss gelangt, dass die bestehende Regulierung auch für Nanomaterialien funktioniert. Sobald man jedoch von Nanoprodukten der nächsten Generation spricht, die komplexere Systeme von Nanostrukturen darstellen, muss man sich eine neue Art der Regulierung überle-

98 Zudem hat der IRGC Konferenzen organisiert, wie die Tagung: ,Risk Governance of Nanotechnology: Recommendations for Managing a Global Issue' vom 6. 7. Juli 2006 am Zentrum für globalen Dialog der Swiss Re bei Zürich (Schweiz). Daraus ist ein Schlussbericht mit Empfehlungen hervorgegangen, welche in etwa denjenigen entsprechen, welche die Royal Society und die Royal Academy of Engineering im Jahr (2004) vorgeschlagen haben (Swiss Re 2006). 
gen. Ich bin Teil des Internationalen Risikoregulierungsrats und wir versuchen, diese Langfristigkeit im Auge zu behalten." (Mitinitiator der NNI, 27. März 2008, Übersetzung der Autorin).

Das vom IRGC entwickelte selbstregulatorische Risikoanalyseschema unterscheidet zwischen verschiedenen Entwicklungsgraden der Nanotechnologie. In seinen Empfehlungen unterscheidet es zwischen den Kategorien ,Gefahr', ,Exposition' und ,Risiko'. Neben den gegenwartsbezogenen und definierbaren Nanomaterialien, erster Generation' zielt es auch auf die Behandlung aktiver Nanostrukturen der sogenannten, zweiten und dritten Generation' ab, deren Form, Struktur, Verhalten und Anwendung als höchst ungewiss gilt (IRGC 2006; IRGC 2007; IRGC 2009). Die Analyse der Dokumente lässt dabei den Eindruck entstehen, dass der damit verbundene Anspruch, für zahlreiche Anspruchsgruppen und unterschiedlichste Anwendungen der Nanotechnologie relevante Ansätze zu entwickeln, das Erreichen eines hohen Detaillierungsgrades erschwert hat. So sind das Schema, wie auch die darauf aufbauenden Empfehlungen, relativ allgemein und übergreifend formuliert (vgl. Abbildung 2). Dabei scheint letztendlich auch für dieses Schema zu gelten, was Grobe, et al. (2008) für zahlreiche weitere selbstregulatorische Risikomanagementansätze festgestellt haben:

„Die verschiedenen freiwilligen Kodizes widerspiegeln auseinanderklaffende regulatorische und kulturelle Hintergründe und zielen auf unterschiedliche Akteure wie die Industrie, Forschende oder gemischte Anspruchsgruppen ab. Derzeit existiert kein gemeinsames Bekenntnis zu keinem dieser Kodizes und es ist fraglich, ob es überhaupt möglich ist, eine Einheitslösung zu finden.“ (Grobe, et al. 2008, 44, Übersetzung der Autorin).

All diese unterschiedlichen Ansätze widerspiegeln divergierende regulatorische und kulturelle Hintergründe und zielen auf unterschiedliche Akteure und Anwendungen ab. Daher bezweifeln Grobe, et al. (2008) die Erfüllbarkeit des Anspruchs, einen allgemeingültigen, international anerkannten Ansatz zu entwickeln, auf den sich sämtliche Länder, Institutionen, Organisationen und Anspruchsgruppen einigen können, wie er im Rahmen solcher Schemen wiederholt geäußert worden ist.

Sowohl auf der OECD-Ebene, wie auch im IRGC, ist die Frage nach der Harmonisierung der bestehenden Regulierung zentral. Die OECD fokussiert dabei insbesondere auf Indikatoren, Definitionen und die Implementierung der unterschiedlichen Gesetze. Demgegenüber scheint der IRGC auch Ideen eines internationalen Abgleichs des Risikomanagements und eine Harmonisierung der Regulierungsansätze zu verfolgen. Dabei 
lässt sich auch hier die doppelte Korrelation feststellen, die sich schon auf Länderebene beim NanoriskFramework, dem Responsible Nanocode und den deutschen Herstellerinitiativen gezeigt hat; je unbestimmter der zu regulierende Gegenstand eingegrenzt wird, desto unverbindlicher wirkt die damit verbundene Lenkungsmaßnahme.

Das bezüglich der Sicherheit von Nanomaterialien bestehende hohe $\mathrm{Ma}$ an Nichtwissen hat zum Dispositiv geführt, die Regulierungsverantwortung auf die internationale Ebene zu delegierien. Diese Strategie scheint nach Aussage verschiedener Interviewpartner bei der Implementierung, also beim Harmonisieren von Testrichtlinien und der Risikoanalyse eine mögliche erfolgsversprechende Option darzustellen. Als weniger tauglich wird sie jedoch bei der Harmonisierung des Risikomanagements und der gesetzlichen Regulierung bewertet. Dennoch - so die These dieser Studie - führt die Delegation der Regulierungsverantwortung auf die internationale Ebene zum Verwischen regulierungskultureller Unterschiede. Dies geschieht nicht auf der Ebene der Anpassung von Gesetzestexten, sondern vielmehr durch die Nichtregulierung der Nanotechnologie auf nationaler stoffrechtlicher Ebene.

Die nachfolgenden Kapitel geben einen länderspezifischen Überblick über den Regulierungsdiskurs der Nanotechnologie. Dieser erfolgt fallweise und exemplarisch. Dabei stehen insbesondere Diskussionen um gesetzliche Maßnahmen, wie die Anwendbarkeit der Umweltgesetzgebung auf Nanomaterialien und die freiwilligen Regulierungsansätze von Regierungsorganisationen im Zentrum der Analyse. Solche umfassen beispielsweise die von den britischen und den US-amerikanischen Umweltbehörden eingesetzten freiwilligen Datenmeldeverfahren, die Ergänzung gesetzlicher Statute im sektoralen Produktrecht, wie dem Lebensmittel- und dem Kosmetikrecht der Europäischen Union, und dem Pestizidrecht in den Vereinigten Staaten. Ein weiterer Fokus der nachfolgenden Länderkapitel liegt auf den selbstregulatorischen Steuerungs- und Lenkungsmaßnahmen, die von regierungsexternen Akteuren entwickelt worden sind, wie prinzipienbasierte und handlungsorientierte Verhaltenskodizes, Risikomanagementschemen, Leitlinien, Richtlinien und Definitionen. Solche werden ebenfalls exemplarisch und beispielhaft analysiert. 


\subsection{Vereinigte Staaten: Freiwilliges Datenmeldeverfahren und unübliche Kooperation}

Dieses Kapitel zum US-amerikanischen Regulierungsdiskurs behandelt einerseits die Frage nach der Behandlung von Nanomaterialien in der bestehenden Umweltregulierung und dabei insbesondere in der Chemikaliengesetzgebung TSCA. Hier steht hauptsächlich das von der Umweltbehörde EPA im Jahr 2008 eingeführt freiwillige Datenmeldeverfahren im Zentrum. Neben einem kurzen Überblick über Regulierungsstrategien weiterer Vorsorgebehörden, wie diejenige der Lebens- und Arzneimittel- (FDA) und der Arbeitssicherheitsbehörde (OSHA und NIOSH) behandelt dieses Kapitel auch von regierungsexternen Akteuren ergriffene Steuerungs- und Lenkungsansätze. Als Beispiel gilt das im Exkurs zum Kapitel 3 besprochene, in der unüblichen Kooperation der Umweltorganisation Environmental Defense Fund mit dem multinationalen Chemikalienhersteller DuPont entstandene NanoRiskFramework. Dieser Fall und die Diskussionen um die gesetzliche Behandlung von Nanomaterialien im Stoffrecht dienen als Beispiele für Praktiken, die im US-amerikanischen Regulierungsdiskurs im Umgang mit dem mit Nanomaterialien verbundenen Nichtwissen ergriffen worden sind.

\subsubsection{Das koordinierte Regelwerk des Nanotechnology Research and Development Act}

Die in den Vereinigten Staaten seit Mitte der 1990er Jahren etablierte umweltpolitische Haltung der Vermeidungsorientierung prägt auch den Regulierungsdiskurs um die Nanotechnologie. Dabei orientiert sich die Strategie der gesetzlichen Behandlung der Nanotechnologie vergleichbar zu derjenigen der Gentechnik an einem koordinierten Vorgehen (vgl. Marchant, et al. 2007). So wird im Jahr 2003 die nanoskalige Forschungs- und Entwicklungsförderung unter dem eigens dafür geschaffenen Nanotechnologieforschungs- und Entwicklungsgesetz (21st Century Nanotechnology Research and Development Act, NRDA) geregelt (P.L. 108-153 2003). Dieses Gesetz behandelt die Finanzierung der nanotechnologischen Forschung und Entwicklung, den Aufbau von behördenübergreifenden begleitenden und organisatorischen Strukturen, die Integration von sozialen Anliegen sowie die Förderung der Bürgerbeteiligung. Mit dem Inkraftsetzen des Gesetzes wird der Nationale Nanotechnologieberatungsausschuss (Na- 
tional Nanotechnology Advisory Panel, NNAP) eingesetzt. Aufgabe dieses forschungspolitischen Gremiums ist die Überwachung und die Koordination der nationalen Forschungs- und Entwicklungsprogramme. Wie das koordinierte Rahmenwerk zur Regulierung der Biotechnologie (Coordinated Framework for Regulation of Biotechnology,) basiert auch die Nationale Nanotechnologieinitiative (NNI) und das daraus entwickelte Nanotechnologieforschungs- und Entwicklungsgesetz (NRDA) auf einem inkrementellen Ansatz. Dies bedeutet, dass die Regulierung der Nanotechnologie im Rahmen der bestehenden Gesetzgebung erfolgt und auf dem Konsens basiert, dass keine neuen Gesetze entwickelt werden sollen (P.L. 108-153 2003). Der 21st Century Nanotechnology Research and Development Act, wird im Jahr 2009 durch den National Nanotechnology Initiative Amendments Act ergänzt, um die Reichweite des nationalen Nanotechnologie Programms auszudehnen. In Unterstützung des Programms soll durch dieses Gesetz die Entwicklung standardisierter Referenzmaterialien, die Teilnahme an der Entwicklung von Standards in nationalen und internationalen Organisationen, die Finanzierung der Nanotechnologieausbildung und Personalentwicklungsprogramme und die Koordination der Forschung ermöglicht werden. Im Jahr 2009 erlässt der U.S. Senat den Nanotechnology Innovation and Price Competition Act. Dieser verpflichtete das Handelssekretariat ein Wettbewerbsprogramm zu institutionalisieren, das finanzielle und ideelle Anreize für Individuen und Firmen schafft. Dabei sollen Entwicklungen der Nanotechnologie in den Bereichen Umwelt, Entwicklung alternativer Energieträger, Verbesserung der menschlichen Gesundheit oder Konsumprodukte gefördert werden. Das Programm wird dem Senatskommittee für Handel, Wissenschaft und Transport unterstellt (Marchant, et al. 2007).

Die im NRDA vorgegebene strukturelle Verflechtung der Förderung der Technikentwicklung mit der Risikoanalyse und der Forschung über die sozialen Implikationen führt zu einer Gleichzeitigkeit zweier antagonistischer Auflagen: So soll die NNI gleichzeitig die Technologie fördern und ihre potenziellen Risiken verhindern. Dies wird als eines der zentralen Probleme des US-amerikanischen Regulierungsansatzes identifiziert. Dazu erläutert ein Interviewpartner einer Umweltorganisation, dass die rechtliche Behandlung von Risikofragen im Rahmen eines Gesetzes zur Technologieförderung schwierig sei. Damit wird die Risikoregulierung als innovationshemmend dargestellt, was eine Sichtweise ist, die selbst von der Industrie nicht geteilt wird: 
„Unsere nationale Nanotechnologieinitiative unterliegt dem grundsätzlichen Konflikt, dass sie die Nanotechnologie nicht nur fördern, sondern auch überwachen muss. Wir haben eine Trennung dieser beiden Funktionen verlangt, da wir davon überzeugt sind, dass die Regierung Risikofragen als Hindernis der Technologieentwicklung sieht. Demgegenüber denken wir und nicht wenige Firmen, dass die Technologie anfälliger wird für die Ablehnung durch die Konsumenten, wenn wir nicht einen Ansatz entwickeln, um mit diesen Risiken umzugehen. In dieser Situation denke ich, dass die Poliitik in der Wahrnehmung des Regulierungsbedarfs noch weiter zurückliegt als die Industrie." (Chemikalienspezialist, Environmental Defense Fund, 6. März 2008, Übersetzung der Autorin).

Im Unterschied zu den anderen untersuchten Ländern wird im US-amerikanischen Regulierungsdiskurs weniger mit dem hohen Maß an Nichtwissen argumentiert, das eine gesetzliche Regulierung verunmöglicht. Ebenso wenig wird argumentiert, dass mit einer freiwilligen Maßnahme die zeitintensive Phase der Entwicklung einer gesetzlichen Regulierung überbrückt werden kann. Vielmehr beobachten verschiedene Interviewpartner eine grundsätzliche Regulierungsunwilligkeit der in den Jahren 2001-2009 regierenden republikanischen Administration unter Päsident George W. Bush und machen für die fehlende Regulierung seine neoliberale Politik verantwortlich. Eine Verantwortliche des Wilson-Zentrums erläutert, dass diese Regulierungsunwilligkeit sämtliche politischen Schritte umfasst; sogar freiwillige Maßnahmen. Das Interview ist unmittelbar vor der Einführung des freiwilligen Datemeldeprogramms (NMSP) der EPA geführt worden. Wie sich aus dem Interviewzitat zeigt, haben damals verschiedene Akteure nicht mehr an dessen Realisierung geglaubt:

„Bis jetzt hat die Regierung sämtliche politischen Schritte vermieden. Sogar die Umweltschutzbehörde ist bislang vor einem freiwilligen Meldeverfahren zurückgeschreckt." (Nanotechnologieverantwortliche, Woodrow Wilson International Center for Scholars, 15. Januar 2008, Übersetzung der Autorin).

Ein Vertreter des Meridian-Instituts stellt einen Zusammenhang zwischen Regulierungsansätzen und der parteilichen Zugehörigkeit der regierenden Administration her. Seiner Meinung nach entscheidet diese darüber, welcher Regulierungsansatz verfolgt wird:

„Die nächste Regierung, ob sie nun republikanisch oder demokratisch wird, entscheidet über die grundsätzliche Richtung und Haltung, die sie in Regulierungsfragen einnimmt. Ich vermute, dass bis zum Ende dieser Administration in einem Jahr, keine regulatorischen Schritte mehr ergriffen werden." (Leitungsmitglied, Meridian Institute, 23. Januar 2008, Übersetzung der Autorin). 
Dabei wird nicht nur die Regulierungsunwilligkeit der damaligen Administration unter George W. Bush problematisiert, sondern auch die in seiner Amtszeit erfolgte Kürzung der ohnehin schon knapp bemessenen Mittel der US-amerikanischen Vorsorgebehörden. Nach Aussage verschiedener Interviewpartner verunmöglicht dies den Behörden, ihre regulatorische Verantwortung wahrzunehmen. So äußert ein Interviewpartner des Wilson-Zentrums seine Zweifel darüber, wie Behörden, die kaum über genügend Mittel verfügen, laufende Bereiche wie Gentechnik und Chemikalien ausreichend $\mathrm{zu}$ behandeln, in der Lage sein sollen, neue, vielschichtige Themen wie die Nanotechnologie zu regulieren:

„Nano ist komplex. Vieles findet im Zwischenbereich zwischen Chemie und Physik und Biologie statt. Die meisten Behörden haben nicht einmal die Kapazitäten, ihre regulatorische Verantwortung wahrzunehmen und den politischen Willen durchzusetzen. So scheitert hier die staatliche Aufsicht." (Leitungsmitglied, Woodrow Wilson International Center for Scholars, 4. März 2008, Übersetzung der Autorin).

Im Rahmen ihres inkrementellen Ansatzes sieht die US-amerikansche Regierung die regulatorische Behandlung der potenziell nachteiligen Implikationen der Nanotechnologie in den bestehenden Strukturen vor. Dabei ist insbesondere das unter der Aufsicht der Umweltbehörde EPA stehende Chemikalienrecht TSCA bedeutsam. Wichtig ist auch das durch die Nahrungs- und Heilmittelbehörde FDA beaufsichtigte Lebensmittelrecht und das Arbeitsrecht, das unter der Aufsicht der Arbeitssicherheitsbehörden OSHA und NIOSH steht. Der Ansatz, Nanotechnologie in den existierenden, divergierenden Strukturen zu regulieren, wird ambivalent diskutiert: Proponenten der inkrementellen Regulierung vertreten die Haltung, dass diese den zuständigen Behörden einen umfassenden Handlungsspielraum eröffnet. In dessen Rahmen können die Behörden die Nanotechnologie innerhalb der in ihren Kompetenzen liegenden Statute in eigener Regie regulieren. Auch können sie ihre regulatorischen Einschätzungen und Entscheidungen untereinander abstimmen (vgl. Marchant, et al. 2007, 190).

Opponenten vertreten hingegen die Ansicht, dass daraus eine grundsätzliche Ungleichbehandlung der verschiedenen Anwendungen resultiert: Während Nanomaterialien in Pharmaka, Medizinalprodukten oder in Pestiziden als wirksam reguliert gelten, werden sie in Bereichen wie Chemikalien, Kosmetika oder Lebensmittelzusatzstoffe als deutlich ,unterreguliert' beschrieben. Dieser Widerspruch zieht sich teilweise mitten durch den Kompetenzbereich der einzelnen Ämter. So gelten im Gegensatz zu TSCA die unter dem FIFRA Statut regulierten Nanotechnologieanwen- 
dungen als ausreichend reguliert, wie ein Interviewpartner des WilsonZentrums feststellt: Während er das Pestizidgesetz als wirksam einschätzt, stellt er der Chemikalienregulierung TSCA ein vergleichsweise schlechtes Zeugnis aus:

„Bezüglich der EPA: TSCA ist aktuell ein sehr schwaches Gesetz, verglichen mit dem FIFRA, den wir auf Pestizide anwenden. Wenn man Nano in Pestizide einbringt, hat man vor der Vermarktung ein rigoroses Testregime zu befolgen. Dasselbe mit Brennstoffzusätzen.” (Leitungsmitglied, Woodrow Wilson International Center for Scholars, 4. März 2008, Übersetzung der Autorin).

Eine vergleichbare Situation zeigt sich auch bei der Lebensmittel- und Arzneimittelbehörde FDA. So liegt deren Kompetenz in der Regulierung von Nanomaterialien in Arzneimitteln, Kosmetika und in Lebensmitteln. Während derselbe Interviewpartner das von der FDA überwachte Arzneimittelgesetz als wirksam einschätzt, identifiziert er signifikante Regulierungslücken in den ebenfalls unter der Zuständigkeit der FDA liegenden Bereichen Kosmetika und Lebensmittel. So sind seiner Meinung nach nanotechnologische Kosmetika und Lebensmittelzusatzstoffe komplett unterreguliert:

„Da gibt es eine Reihe von Defiziten im System. Wenn man die Lebensmittel und Arzneimittelbehörde anschaut, dann denke ich, dass ihre Fähigkeit mit Nano in medizinischen Hilfsmitteln und Medikamenten umzugehen, möglicherweise in Ordnung ist. Sie sind seit sieben Jahren gezwungen, mit der Industrie zusammenzuarbeiten und Versuche zu machen. Das ist die Vorvermarktungsinteraktion mit der Industrie. Das alles gibt es nicht mit Kosmetika oder Lebensmittelzusatzsstoffen. Diese Produkte stehen einfach so in den Regalen." (Leitungsmitglied, Woodrow Wilson International Center for Scholars, 4. März 2008, Übersetzung der Autorin).

Wie derselbe Interviewpartner weiter erläutert, liegen Regulierungsdefizite häufig ausgerechnet in den Bereichen, in welchen die meisten Nanotechnologieprodukte vermarktet werden:

„Nicht das Regulierungssystem selbst ist unausreichend, es ist lediglich die Tatsache, dass es in bestimmten Bereichen große Löcher gibt. Was uns Sorgen macht, ist, dass sich diese Bereiche genau dort befinden, wo viele Nanoprodukte auf den Markt gebracht werden." (Leitungsmitglied, Woodrow Wilson International Center for Scholars, 4. März 2008, Übersetzung der Autorin).

Im Gegensatz zur EPA wird die FDA im politischen Diskurs um die Regulierung von Nanomaterialien weniger stark kritisiert. Ebenfalls im Unterschied zur EPA lassen sich bei der FDA verschiedene Ansätze der Kooperation mit unterschiedlichen Anspruchsgruppen inklusive partizipatorische 
Ansätze zur Einbindung der Zivilgesellschaft beobachten (siehe Kapitel 4). Dennoch wird auch der regulatorische Ansatz der FDA kritisiert. Insbesondere problematisiert wird ihr produktbasierter Regulierungsansatz. Dieser führt einerseits zu einer hohen regulatorischen Divergenz zwischen einzelnen Produkten. Beispielsweise werden für Medikamente umfangreiche Vormarktsetzungstests verlangt, während Kosmetika ohne solche auf den Markt gebracht werden können.

Dadurch und durch die Schwierigkeit, aktive Nanostrukturen höherer Generationen einer einzelnen Produktkategorie zuzuordnen, ergeben sich zusätzliche regulatorische Probleme (vgl. Marchant, et al. 2007). Zudem hat die FDA die im Arbeitsgruppenbericht (Alderson, et al. 2007) zugesagten wissenschaftlichen Analysen der potenziellen Umwelt-, Gesundheits- und Sicherheitsimplikationen von Nanomaterialien, die ihren Regulierungsbereich betreffen, nicht weitergeführt. Dies hat ihr zusätzliche Kritik eingebracht. Wie der Interviewpartner des Wilson-Zentrums erläutert, vermisst er Informationen darüber, wie die FDA die regulatorischen Implikationen derzeit einschätzt:

„Sie haben versprochen, auf diese Punkte zu reagieren, aber es kam nie etwas. Wir wissen, dass die FDA wirklich mit der Nanomaterialienregulierung vorankommen will. Aber wir wissen nicht, wie sie genau vorgehen und wohin es führen wird." (Nanotechnologieverantwortlicher, Woodrow Wilson International Center for Scholars, 15. Januar 2008, Übersetzung der Autorin).

Im Jahr 2010 erlässt der US-amerikanische Senat das Nanotechnologiesicherheitsgesetz (Nanotechnology Safety Act). Dieses Gesetz betrifft die FDA, die verpflichtet wird, ein Programm zur Untersuchung von Nanomaterialien in Arzneimitteln, Kosmetika und Lebensmitteln zu entwickeln (P.L. S 2942 2010). Weitere regulatorische Ansätze betreffen beispielsweise die im Jahr 2010 publizierten Richtlinien der NASA zum Umgang mit Nanomaterialien in eigenen Forschungslaboratorien (vgl. Shapira \& Youtie 2010).

Die Arbeitssicherheitsbehörden OSHA und NIOSH verfolgen ebenfalls einen vermeidungsorientierten Regulierungsansatz. Im Unterschied zu den europäischen Arbeitssicherheitsstandarts, die als Vorsorgemaßnahme etabliert werden, können im Rahmen des US-amerikanischen Ansatzes lediglich bei wissenschaftlich nachgewiesenen Schädigungen Grenzwerte etabliert werden. Dabei vertritt insbesondere die NIOSH die Haltung, dass Nanomaterialien ein Gefahrenpotenzial für die menschliche Gesundheit darstellen und dass angesichts dieser Gefahren ein vorsorgeorientierter 
Regulierungsansatz zu prüfen ist. ${ }^{99}$ Im Unterschied zu NIOSH gilt die Partnerbehörde OSHA in Fragen der Nanotechnologieregulierung als relativ zurückhaltend (vgl. Marchant, et al. 2007). Ein Grund dafür wird in der Beweislast gesehen, welche das Amt bei der Festlegung von Grenzwerten trägt, wie ein Interviewpartner der OSHA erläutert:

„Neben dem Setzen von Standards, liegt eine Aufgabe der OSHA darin, Richtlinien und Informationen bereitzustellen, die den Verantwortlichen hilft, Arbeitsplatzrisiken anzugehen. Die OSHA hat neue Standards für Chemikalien gesetzt, diese betreffen auch die Nanotechnologie. Dabei liegt jedoch eine relativ hohe Beweislast bei der Behörde." (Nanotechnologieverantwortlicher, OSHA, 15. Januar 2008, Übersetzung der Autorin).

Der Interviewpartner erwähnt, dass derzeit keine spezifischen Grenzwerte für Nanomaterialien existieren. Wenn Nanoformen bestimmter Chemikalien in relevanten Mengen produziert werden, fallen sie unter die bestehenden Chemikalienstandards. Falls jedoch Daten publiziert werden, die auf eine potenzielle Gefährdung hinweisen, kann die OSHA umgehend Standards setzen:

„Wir haben bislang noch keine Standards entwickelt, welche die spezifischen Eigenschaften von Nanomaterialien betreffen. Wir haben aber eine Menge allgemeiner Standards, die für alle gefährlichen Chemikalien gelten, die sich im Handel befinden. Diese schließen Nanomaterialien mit ein. Dennoch haben wir auch Werkzeuge, die es der OSHA erlauben, Standards durchzusetzen, sobald wir über Daten verfügen, die auf potenzielle Gefahren hinweisen." (Nanotechnologieverantwortlicher, OSHA, 15. Januar 2008, Übersetzung der Autorin).

Im vermeidungsorientierten Kontext der Vereinigten Staaten, in dem dann gesetzlich reguliert wird, wenn, wissenschaftlich gesicherte' Nachweise einer Gefährdung vorliegen (siehe Kapitel 3.2), überrascht die eher vorsorgeorientierte Sprachwahl des Interviewpartners, der von ,Hinweisen' auf ,potenzielle Gefahren's spricht.

99 Da die NIOSH selbst über keine Regulierungsautorität verfügt, kann sie lediglich Empfehlungen abgeben. Über deren Umsetzung in Standards entscheidet die OSHA. Verschiedene Beobachter gehen davon aus, dass die NIOSH ihren regulatorischen Einfluss alleine durch wissenschaftliche Integrität und Exzellenz verstärken kann. Aus diesem Grund ist es kaum denkbar, dass die NIOSH ohne signifikante wissenschaftliche Grundlagen substanzielle Empfehlungen zur Arbeitssicherheit und der Toxizität von Nanomaterialien abgibt (vgl. Marchant, et al. 2007, 199). 
Die US-amerikanische Regulierungskultur in den Bereichen Umwelt, Chemikalien und Gesundheit sieht einen wissenschaftlichen Nachweis der Gefährdung eines bestimmten Stoffes oder einer Anwendung vor, bevor diese reguliert werden kann. Zudem sind mit der Festlegung von Expositionsgrenzwerten hohe Schwierigkeiten verbunden. Erst muss eine Fülle an Wissen generiert werden, wie die detaillierte Analyse der vorhandenen wissenschaftlichen Studien bezüglich Risiken, Reaktivität und Exposition bestimmter Stoffe. Zudem müssen Risikoanalysen durchgeführt und wissenschaftliche Unsicherheiten berücksichtigt werden. Auch muss die Behörde das Risiko für Arbeitskräfte abschätzen, die Machbarkeit der Implementierung unterschiedlicher Kontrollmaßnahmen nachweisen und ökonomische Effekte mitberücksichtigen:

„Wir müssen die wissenschaftliche Grundlage der Risikoinformation detailliert untersuchen. Wir müssen Risikoabschätzungen machen, Frequenzgangkurven analysieren, wissenschaftliche Unsicherheiten berücksichtigen und eine vertiefte Analyse machen, um herauszufinden, was die Expositionsgrenze sein soll. Wir müssen das Risiko für die Arbeitnehmer abschätzen, die Machbarkeit der Implementierung verschiedener Kontrollmaßnahmen einschätzen. Wir müssen ökonomische Effekte und Auswirkungen, insbesondere Auswirkungen auf kleine Unternehmen beachten. Wir müssen all diese Aspekte mitberücksichtigen." (Nanotechnologieverantwortlicher, OSHA, 15. Januar 2008, Übersetzung der Autorin).

Die daran anschließenden wissenschaftlichen Diskussionen nehmen ebenfalls viel Zeit und Ressourcen in Anspruch. Deshalb liegt die Strategie der OSHA darin, nur für akut toxische Substanzen Grenzwerte zu setzen:

„Anschließend folgt eine Reihe wissenschaftlicher Argumente, die sehr viel Zeit und Ressourcen bindet. Da die Entwicklung eines Grenzwertes so viel Arbeit in Anspruch nimmt, wollen wir primär Standards entwickeln, die einen hohen Nutzen haben und Arbeitnehmerinnen und Arbeitnehmer effektiv schützen. Folglich haben wir über die Jahre nicht sehr viele neue chemische Standards gesetzt. Wir versuchen uns auf sehr große Gefahrenexpositionen zu konzentrieren." (Nanotechnologieverantwortlicher, OSHA, 15. Januar 2008, Übersetzung der Autorin).

Ein Interviewpartner erläutert, dass das $\mathrm{Ma} ß$ an Nichtwissen noch zu hoch ist, um entscheiden zu können, ob Nanomaterialien spezifisch reguliert werden müssen. So liegen zu wenig wissenschaftliche Daten vor, die Risiken sind zu wenig geklärt und die Toxizität der Nanomaterialien ist unklar. Zudem ist sich die Behörde nicht im Klaren darüber, welche Arbeitsschutzmaßnahmen am wirkungsvollsten sind. Die derzeitige Strategie der Behörde im Umgang mit diesem Nichtwissen liegt daher in der Kooperati- 
on mit ihrer Partnerbehörde NIOSH und der britischen Gesundheitsbehörde HSE. Von der britischen Gesundheitsbehörde soll das COSHH-Klassifizierungsschema übernommen werden. ${ }^{100}$ Dieses ermöglicht der OSHA in Kooperation mit der NIOSH, eine Risikoeinschätzung aufgrund standardisierter Struktur- und Expositionsmerkmale durchzuführen und für besonders gefährliche Prozesse Kontrollblätter zu entwickeln:

„Wir versuchen mit der NIOSH zusammen Kontrollblätter zu entwickeln, die sich am COSHH System der britischen Gesundheitsbehörde orientieren. Dies wird als Kontrollbindung bezeichnet. Wir konzentrieren uns vorerst auf Prozesse, die mit höchster Wahrscheinlichkeit luftübertragene Emmissionen verursachen. Also wird es einige der heißen Prozesse betreffen. Darüber hinaus könnten wir weitere, damit verbundene Gefahren anschauen." (Nanotechnologieverantwortlicher, OSHA, 15. Januar 2008, Übersetzung der Autorin).

Im Kontext des mit der Nanotechnologie verbundenen hohen Maßes an Nichtwissen, dient die Kooperation mit dem NIOSH und weiteren Behörden primär der Klärung des Forschungsbedarfs. Das nachfolgende Interviewzitat steht für die vielfältigen Unklarheiten, Unsicherheiten und das hohe Maß an Nichtwissen bezüglich der mit den Nanomaterialien verbundenen Gefahren:

„Für die OSHA liegen noch zu wenig wissenschaftliche Daten vor, um darüber zu entscheiden, ob wir einen Grenzwert benötigen, der die Exposition zu diesem Material einschränkt. Wir können das noch nicht sagen. Wir sind noch zuwenig sicher, wie ein solches Risiko aussieht. Wir wissen noch nicht genug über die Charakteristika von Nanomaterialien, über deren Toxizität und das Risiko. Wir sind noch nicht einmal sicher, welches die besten Maßnahmen hinsichtlich der Arbeitsplatzexposition sind. Als Ergebnis haben wir mit der NIOSH und weiteren nationalen Behörden zusammengearbeitet, um herauszufinden, welche Art von Forschung durchgeführt werden muss." (Nanotechnologieverantwortlicher, OSHA, 15. Januar 2008, Übersetzung der Autorin).

Die OSHA bestimmt in Abstimmung mit der NIOSH die durchzuführende Forschung, von der die OSHA mangels eigener Forschungskompetenz, abhängig ist. Der Interviewpartner erläutert sein Interesse an einem ra-

100 Das COSHH-Klassifizierungsschema steht für die von der britischen Gesundheitsbehörde Health and Safety Executive entwickelten Kontrollblätter für gesundheitsgefährdende Stoffe (Control of Substances Hazardous for Health). Diese umfassen Richtlinien und Vorschriften (vgl. http://www.hse.gov.uk/pubns/indg 136.htm/ 13.05.2014). 
schen Vorliegen von Ergebnissen, damit sie darauf basierend Empfehlungen, Arbeitsschutzbestimmungen und Grenzwerte festsetzen können:

„Wir arbeiten mit der NIOSH zusammen. Sie haben uns die Daten zur Verfügung gestellt und wir versuchen basierend darauf, eine Handlungsanweisung für Hersteller dieser Materialien zu verfassen. Aus dieser Forschung ergeben sich also Empfehlungen. Denn Empfehlungen und Handlungsanweisungen können wir sehr schnell machen. Grenzwerte dauern etwas länger. Aber wir wollen nicht warten. Wenn wir die Information haben, die uns hilft, Maßnahmen zu entwickeln, welche die Exposition reduzieren, möchten wir, dass diese Maßnahmen sofort umgesetzt werden.” (Nanotechnologieverantwortlicher, OSHA, 15. Januar 2008, Übersetzung der Autorin).

Die in der Regulierung der Nanotechnologie durch die Vorsorgebehörden FDA und OSHA festgestellten Defizite, Schwierigkeiten und Unsicherheiten betreffen auch die Umweltbehörde EPA, wie das nachfolgende Kapitel zeigt.

\subsubsection{Nanomaterialien in der Umwelt- und Chemikalienregulierung}

Als die für die Umwelt- und Chemikalienregulierung zuständige Behörde, ist die EPA zentral in den Regulierungsdiskurs der Nanotechnologie involviert. Ihre Aktivitäten betreffen die Förderung der Forschung und den Aufbau von internationaler Expertise in umweltbezogenen Anwendungen und Implikationen der Nanotechnologie (vgl.Marchant, et al. 2007, 192). Zudem stehen Fragen wie solche nach der Zuständigkeit der Chemikaliengesetzgebung TSCA (siehe Kapitel 3.1) für die Nanotechnologie im Zentrum ihrer behördlichen Tätigkeiten. Im Regulierungsdiskurs etwas weniger prominent vertreten sind die medienspezifischen Gesetze, wie das Luftreinhaltegesetz (Clean Air Act, CAA), das Wasserreinhaltegesetz (Clean Water Act, CWA), das Ressourcenschutz und Rückgewinnungsgesetz (Resource Conservation and Recovery Act, RCRA) und das Pestizidgesetz (Federal Insecticide, Fungicide and Rhodendicide Act, FIFRA).

Bei der Regulierung von Nanomaterialien unter dem traditionellen Chemikalienstatut TSCA wendet die EPA den im Stoffrecht etablierten Strukturäquivalenzansatz auch auf Nanomaterialien an. Dieser Ansatz ermöglicht eine Gefährdungseinschätzung von Chemikalien abhängig ihrer molekularen Struktur. Da die Reaktivität von Nanomaterialien größenabhängig eintritt, gilt die Anwendung dieses Ansatzes als problematisch (vgl. Denison 2007). Zudem werden die Chemikalien in Alt- und Neustoffe klassiert. Für die sich bereits auf dem Markt befindenden Altstoffe müssen 
keine Sicherheitsnachweise erbracht werden. Die Sicherheitsnachweise für die als Neustoffe klassierten Nanomaterialien erfolgen aufgrund strukturbasierter Analysen und damit verknüpften hypothetischen Erwartungswerten bezüglich ihrer Reaktivität (vgl. Kapitel 3.2). Die regulatorische Behandlung von Nanomaterialien, unter dem auf Stoffe mit eindeutiger chemischer Identität zugeschnittenen Chemikalienstatut TSCA, wird von verschiedenen Interviewpartnern kritisiert.

Der EPA wird vorgeworfen, durch ihren ausschließlichen Fokus auf die molekulare Struktur anstelle der Reaktivität, chemische Substanzen zu eng zu definieren. Damit werden die spezifischen Eigenschaften von nanoskaligen Materialien, die trotz der gleichen chemischen Identität andere Reaktionen zeigen, ignoriert. Dies führt dazu, dass chemisch identische Substanzen unterschiedlicher Größenordnung mit verschiedensten Eigenschaften irrtümlich als sicher bewertet werden. Zudem fehlt der Behörde die Möglichkeit, diese Materialien vor Markteintritt zu testen. Der schon bei Makroformen umstrittene Ansatz der EPA, Chemikalien mittels Äquivalenzschlüsse aufgrund morphologischer Ähnlichkeiten zu regulieren, wird insbesondere bei Nanoformen als ungeeignet beschrieben (ibid.). Dies zeigt sich auch in den Interviews. Dazu erläutert ein Interviewpartner des Wilson-Zentrums, dass Stoffe in diesem Größenbereich völlig unvorhersehbar reagieren. Im Nanogrößenbereich werden Reaktivitäten in erster Linie durch die Skalengröße beeinflusst und weniger durch Strukturmerkmale oder Stoffidentitäten:

„TSCA wurde entwickelt, um Stoffe mit gleicher chemischer Identität zu regulieren, wie Atome und Moleküle. Dies funktioniert jedoch nicht bei Stoffgemischen, wie dies bei synthetischen Materialien der Fall ist. Bei intendiert hergestellten Nanomaterialien kann die chemische Identität dieselbe sein, aber das Verhalten und das Risiko können stark abweichen." (Nanotechnologieverantwortlicher, Woodrow Wilson International Center for Scholars, 15. Januar 2008, Übersetzung der Autorin).

Die regulatorische Schlagkraft von TSCA wird insbesondere wegen der bei der Behörde liegenden Beweislast als enttäuschend bezeichnet und ihre Eignung für die Regulierung von Nanomaterialien infrage gestellt (vgl. Marchant, et al. 2007). Zudem werden Bedenken gegenüber der dem Stoffrecht zugrunde liegenden Volumenabhängigkeit geäußert. Substanzen, die in einem Volumen von weniger als elf Tonnen pro Jahr produziert werden, sind von der Regulierung unter TSCA ausgenommen und bleiben unreguliert. Da verschiedene Nanomaterialien auch in geringen Mengen hoch reaktiv sind, wird dieser massenbasierte Grenzwert insbesondere bei 
der Regulierung von Nanomaterialien als problematisch kritisiert (ibid.). Diese Problematik wird auch in den Interviews angesprochen. So erläutert ein Interviewpartner des Wilson-Zentrums, dass Nanomaterialien bereits in sehr viel geringeren Konzentrationen toxisch sind:

„Ein grundlegendes Defizit im regulatorischen System liegt darin, dass die meisten unserer Gesetze massenbasiert oder grenzwertorientiert sind. Und wir können das mit Nano nicht so machen. Mit Nano ist die Toxizität an die Oberfläche, die Struktur oder die Morphologie gebunden und deshalb liegen hier inhärent eine geringe Masse und ein hohes Volumen vor. Damit werden Grenzwerte erst dann erreicht, wenn jemand fast zehntausend Tonnen dieses Materials emittiert. So kann man unter den derzeitigen Grenzwertgesetzen eine vollständige Industrie unreguliert aufbauen." (Leitungsmitglied, Woodrow Wilson International Center for Scholars, 4. März 2008, Übersetzung der Autorin).

Verschiedentlich wird in den Vereinigten Staaten von einem grundsätzlichen Defizit in der regulatorischen Behandlung der Nanotechnologie gesprochen. Dazu vertreten verschiedene Interviewpartner die Haltung, dass die Regierung in den letzten acht bis zehn Jahren ihre Aufsichtspflicht kaum wahrgenommen hat. Dadurch ist nach Aussage desselben Interviewpartners eine Regulierungslücke entstanden:

„In diesem Land nimmt die Regierung ihre Aufsicht seit den letzten 8-10 Jahren kaum mehr wahr. So ist ein politisches Vacuum entstanden." (Leitungsmitglied, Woodrow Wilson International Center for Scholars, 4. März 2008, Übersetzung der Autorin).

Als eine zentrale Strategie der EPA im Umgang mit dem mit diesen Fragen verbundenen Nichtwissen etabliert die Behörde ein freiwilliges Datenmeldeschema. Das Ende Januar 2008 von der EPA eingeführte freiwillige Datenmeldeverfahren orientiert sich an dem VRS, das die britische Umweltbehörde DEFRA im Vorjahr eingeführt hat. Das Nanomaterialien Aufsichtsprogramm NMSP der EPA ist für eine Dauer von zwei Jahren angelegt. Es wird dem Chemikaliengesetz TSCA zugeordnet und gilt für Hersteller und Verarbeitungsfirmen. Mit dem NMSP sollen Daten generiert werden, um eine wissenschaftliche Begründung für die regulatorische Behandlung von Nanomaterialien zu erhalten (EPA 2008). Das Programm soll die Behörde in der Erfassung von den sich im Verkehr befindenden nanoskaligen Chemikalien und Nanotechnologieprodukten unterstützen. Weiter soll es die Frage beantworten, ob die im TSCA vorgesehene regulatorische Äquivalenzbehandlung von chemischen Substanzen und Nanomaterialien weiterhin ausreichenden Schutz für die menschliche Gesund- 
heit und die Umwelt gewährleistet. Zudem sollen Hersteller- und Vertriebsorganisationen in der Entwicklung nanospezifischer Risikomanagementpraktiken und Testmethoden unterstützt werden.

Das Programm wird als vorsorgeorientierte Maßnahme geplant, um die Zeit zu überbrücken, bis verlässliche Daten vorliegen. Ebenfalls soll es die Zusammenarbeit zwischen der EPA und den involvierten Akteuren insbesondere im Bereich der Datenerhebung fördern (EPA 2008). Die im Rahmen des Programms gesammelten Daten sollen also dazu beitragen, das hohe Maß an Nichtwissen um die Regulierung der Nanotechnologie zu reduzieren. Wie ein Interviewpartner der Umweltbehörde erläutert, soll das Programm die durch widersprüchliche Studien unübersichtlich erscheinende Situation im Bereich der Nanomaterialien klären. Davon verspricht sich der Interviewpartner ein besseres Verständnis der Einflussfaktoren auf die Toxizität von Nanomaterialien. Dies stellt für ihn eine unabdingbare Voraussetzung für sämtliche regulatorische Entscheidungen dar:

„In unseren Augen ist die Schlüsselaufgabe bei den Nanomaterialien das Generieren von Daten. Derzeit ist es schwierig, die verschiedenen Datensets zu verstehen; ob die Nanoform toxischer ist als die Makroform oder ob sie weniger toxisch ist. Kleine Unterschiede in der Durchführung der Tests führen zu höchst unterschiedlichen Ergebnissen. Deshalb ist die Herstellung von vollständigen Datensets, die Forschung und das Verständnis des Einflusses der Toxizität von Nanomaterialien für eine fundierte regulatorische Entscheidungsfindung wirklich entscheidend.“ (Jim Willis, EPA, 22. Januar 2008, Übersetzung der Autorin).

Freiwillige Regulierungsmaßnahmen werden auch aus Effizienzgründen ergriffen. So erläutert derselbe Interviewpartner, dass die Etablierung eines freiwilligen Programms administrativ weniger Zeit und Aufwand benötigt als eine gesetzliche Maßnahme:

„Es ist einfacher oder schneller, ein freiwilliges Programm zu etablieren, als ein regulatorisches Programm. Die Entwicklung eines regulatorischen Programmes dauert vier oder fünf Jahre und ein freiwilliges ein oder zwei Jahre." (Jim Willis, EPA, 22. Januar 2008, Übersetzung der Autorin).

Innerhalb der EPA wird das Programm als Interimsmaßnahme gesehen. So vertritt der Interviewpartner die Überzeugung, dass bei einer tiefen Beteiligungsquote auch ein verbindlicher Ansatz durchgesetzt werden kann:

„Unsere Überlegung ist es, mit einem freiwilligen Programm zu starten. Wenn wir eine tiefe Beteiligung erhalten, werden wir genug gelernt haben, um etwas Verbindlicheres oder eine gesetzliche Regulierung zu etablieren.“ (Jim Willis, EPA, 22. Januar 2008, Übersetzung der Autorin). 
Unmittelbar nach seiner Lancierung im Jahr 2008 wird das NMSP breit kritisiert. Es wird bemängelt, dass die beispielsweise im britischen Royal Society Bericht (RS\&RAE 2004) vorgeschlagene vorsorgeorientierte regulatorische Behandlung der Nanotechnologie nicht berücksichtigt wird. Sowohl das Konzept eines freiwilligen Ansatzes als auch die behördliche Behandlung von Nanomaterialien nicht als Neustoffe, sondern analog zu ihren Makroformen widersprechen diesen Empfehlungen und werden als ungenügend kritisiert (vgl. Bergeson \& Plamondon 2007; Bullis 2008; Hanson 2008). Grundsätzlich konfrontieren die Kritiken die Behörden mit dem Vorwurf, dass sie die potenziellen Implikationen und Gefahren von Chemikalien und Nanomaterialien nicht angemessen berücksichtigen. Die von den verschiedenen Interviewpartnern geäußerte Hauptkritik liegt darin, dass das Programm angesichts der fortgeschrittenen technologischen Entwicklung und der hohen Anzahl der sich bereits auf dem Markt befindenden ungetesteten Produkte zeitlich zu spät eingeführt worden ist. Als die erste gesetzliche Maßnahme, die im Bereich der Nanotechnologie ergriffen worden ist, wird seine inhaltliche Ausformulierung als zu vage kritisiert. Ein Interviewpartner einer Umweltorganisation vermutet, dass das freiwillige Datenmeldeprogramm durch seine späte Einführung lediglich dazu dient, weitergehende Aktivitäten zu vermeiden:

„Dieses Programm hätte vielleicht vor zweieinhalb Jahren Sinn gemacht, aber so haben sie es letztendlich geschafft, es erst im letzten Monat zu lancieren. Wir sind sehr kritisch, weil es zu spät kommt und zu wenig weit geht. Und wir denken nicht, dass es die notwendigen Informationen generieren wird. In verschiedener Hinsicht denke ich, dass es politisch lediglich einen Versuch darstellt, weitere Schritte zu verzögern und aufzuschieben." (Richard Denison, Environmental Defense Fund, 6. März 2008, Übersetzung der Autorin).

Ein Interviewpartner des Wilson-Zentrums vertritt die Haltung, dass die Umweltbehörde über keine Langzeitstrategie zur regulatorischen Behandlung von Nanomaterialien verfügt. Insbesondere vermisst er bei der EPA den politischen Willen, eine effiziente regulatorische Aufsicht auszuüben:

„Die EPA hat gesagt, dass sie dieses freiwillige Meldeprogramm entwickelt, um herauszufinden, wo die Probleme liegen, aber sie hat nicht gesagt, was danach kommt. Sie hat sich nie zu einem Langzeitprogramm oder einer Langzeitstrategie bekannt, innerhalb derer dies ein erster Schritt wäre. Dabei sollte es sich um einen Prozess hinsichtlich einer effektiven regulatorischen Aufsichtsführung handeln. Soweit ich jedoch sehen kann, ist das freiwillige Datenmeldeschema ein guter Schritt voran, es hilft der EPA, die Informationen zu entwickeln. Das Programm kann ein Mittel zu einer abschließenden Beurteilung sein, aber nicht der Abschluss an sich." (Nanotechnologieverantwort- 
licher, Woodrow Wilson International Center for Scholars, 15. Januar 2008, Übersetzung der Autorin).

Zwei grundsätzliche Kritikpunkte an freiwilligen Datenmeldeverfahren betreffen ihre Unverbindlichkeit und den hohen Aufwand, den teilnehmende Firmen mit der Datenmeldung auf sich nehmen müssen. So erläutern verschiedene Interviewpartner die Vermutung, dass sich aufgrund des anspruchsvollen Aufbaus des Meldeschemas kaum eine Firma die Zeit nehmen wird, Daten freiwillig zu melden. Dies betrifft insbesondere Neugründungen und kleine bis mittelgroße Firmen, die hauptsächlich an der Nanotechnologieentwicklung beteiligt sind. Diese verfügen nach Aussage eines weiteren Interviewpartners des Wilson-Zentrums über zu geringe Ressourcen, um sich freiwillig um Umwelt- und Risikoaspekte zu kümmern. Dies wird auch dadurch erschwert, dass die Behörde kaum inhaltliche Unterstützung anbietet:

„Achzig oder neunzig Prozent der Nanofirmen in diesem Land haben weniger als zehn Mitarbeiter. Die meisten Firmen sind sich der potenziellen Risikoaspekte durchaus bewusst, aber sie haben nicht die Ressourcen, um die Daten zu erstellen und sie mühen sich an den fehlenden Anleitungen und Signalen der Regierung ab.“ (Leitungsmitglied, Woodrow Wilson International Center for Scholars, 4. März 2008, Übersetzung der Autorin).

Da das Datenmeldeprogramm freiwillig ist, fühlen sich nur wenige Firmen angesprochen, ihre Daten zur Verfügung zu stellen. Der Mitarbeiter des Wilson-Zentrums vertritt die Meinung, dass für die Firmen jeglicher Anreiz fehlt, mitzumachen, da das Datenmeldeprogramm isoliert eingeführt worden ist. So fehlt hier der Druck, der von einer real bevorstehenden gesetzlichen Regulierung ausgehen würde:

„Es ist offensichtlich, dass unter dieser Regierung keinerlei Bedrohung vorhanden ist, dass irgendetwas reguliert wird. Es gab keine klaren Anreize für Firmen, hier mitzumachen und es ist eine ziemliche Arbeit." (Leitungsmitglied, Woodrow Wilson International Center for Scholars, 4. März 2008, Übersetzung der Autorin).

Eine Wissenschaftlerin der Rice Universität stellt den Vergleich zum britischen Programm her. Dabei äußert sie ihre Bedenken, dass auch der USamerikanische Ansatz nicht das hohe Maß an Teilnahme und an Wissen generiert, wie es sich die EPA erhofft. Dies lässt sich ihrer Ansicht nach nur bei zwingenden Ansätzen durchsetzen:

„Ich befürchte, dass sie weder die erwartete Art der Partizipation noch das $\mathrm{Ma} \beta$ an Information erhalten, bevor sie die Firmen nicht dazu zwingen. In Großbritannien gibt es ebenfalls ein Programm, das DEFRA Programm und 
sie haben ebenfalls Schwierigkeiten, Firmen für eine Teilnahme zu gewinnen. Dies kann darauf hindeuten, dass freiwillige Programme nicht zwingend der beste Ansatz sind.“ (Leitungsmitglied, CBEN, Rice University, 21. Januar 2008, Übersetzung der Autorin).

Bei Abschluss des Programmes im Jahr 2009 hat die EPA Meldung von insgesamt 29 Firmen und 123 Substanzen erhalten (EPA 2009, 3). Diese Meldequote gilt als enttäuschend, da das Nanotechnologiekonsumprodukteinventar des Wilson Zentrums in diesem Zeitraum von 743 sich auf dem Markt befindenden Nanotechnologieprodukten ausgeht. ${ }^{101}$ Nach Aussage der EPA ist das Nichtwissen nach wie vor hoch und es verbleiben auch nach dem Programmabschluss eine Reihe von Datenlücken im Bereich der Umwelt-, Gesundheits- und Sicherheitsimplikationen von Nanomaterialien (EPA 2009). Um diesen zu begegnen hat die EPA die Datenmeldefrist bis Ende Januar 2010 verlängert. Zudem hat sie TSCA im Jahr 2010 durch einen vierten Abschnitt mit Vorfabrikationsmeldungen und verpflichtenden Richtlinien zur Test- und Informationspflicht ergänzt. Hersteller bestimmter Nanomaterialien sollen dazu verpflichtet werden, Gesundheitstests durchzuführen und die Ergebnisse der EPA zu kommunizieren. Weitere Anpassungen sind im Abschnitt 8(a) geplant, worin Hersteller bestimmter Nanomaterialien gesetzlich zur Datenlieferung gezwungen werden können. Zudem soll der Abschnitt 5(a)(2) angepasst werden, der diese Nanomaterialien unter eine, signifikant neue Verwendungsregel' (Significant New Use Rules, SNUR) stellt (EPA 2009). ${ }^{102}$ Neben dem freiwilligen Datenmeldeprogramm und den damit verknüpften Regulierungsschritten ergreift die EPA auch gesetzliche Maßnahmen für spezifische Nanomaterialien. So reguliert sie beispielsweise Nanosilberpartikel in Samsung Waschmaschinen seit dem Jahr 2008 als Pestizide unter dem nationalen Pestizidgesetz (Federal Insecticide, Fungicide and Rhodendicide Act, FIFRA) (P.L. 113-100 2012).

Das von verschiedenen Akteuren festgestellte grundsätzliche Defizit in der regulatorischen Behandlung der Nanotechnologie in den Vereinigten Staaten trägt dazu bei, dass lokale Behörden einzelner Bundesstaaten oder Gemeinden individuelle Regulierungsmaßnahmen ergreifen, wie die Gemeinde Berkeley, die ein eigenes Nanotechnologiegesetz in Kraft gesetzt

101 Zum Nanotechnologiekonsumprodukteinventar des Wilson Zentrums siehe http:// www.wilsoncenter.org/article/inventory-finds-increase-consumer-products-contai ning-nanoscale-materials (18.02.2014).

102 Siehe dazu http://www.epa.gov/oppt/nano/index.html\#snur (21.04.2014). 
hat (vgl. Lerer 2013). ${ }^{103}$ Zudem entwickeln verschiedene regierungsexterne Akteure selbstregulatorische Maßnahmen. Beides wird als problematisch bezeichnet. Wie der Leiter des Wilson-Zentrums erläutert, führen lokale oder staatliche Initiativen zu Anpassungsschwierigkeiten für national agierende Firmen. Vergleichbar zu den Problemen, die globalen Konzernen aus transnationalen Regulierungsdifferenzen zwischen den Vereinigten Staaten und der Europäischen Union erwachsen, führen kommunale oder staatliche Regulierungen für die nationalen Unternehmen zu Schwierigkeiten:

„Berkeley Kalifornien, Cambridge Massachusetts, der Bundesstaat Wisconsin, sie alle haben laufende Verfahren im Bereich der Nano. Das führt zu Schwierigkeiten in der Industrie. Dies führt zur Trennung von Märkten. Alleine schon die unterschiedlichen Systeme der Vereinigten Staaten und Europas stellen multinationale Firmen vor Schwierigkeiten." (Leitungsmitglied, Woodrow Wilson International Center for Scholars, 4. März 2008, Übersetzung der Autorin).

Gegenstand des nächsten Kapitels sind Initiativen außerhalb der Politik und von Regulierungsbehörden entstehende Initiativen wie das im Exkurs zum Kapitel 3 vorgestellte, in der Kooperation der Umweltorganisation Environmental Defense Fund mit der multinationalen Chemiefirma DuPont entwickelte Nano Risk Framework. Dabei liegt der Fokus auf Fragen, wie es zu einer solchen Partnerschaft gekommen ist und welche Bedeutung das Nano Risk Framework im US-amerikanischen Regulierungsdiskurs spielt.

\subsubsection{Private Initiativen: Das EDF - DuPont ,Nano Risk Framework'}

Die nachfolgende Analyse des Nano Risk Framework zeigt, dass insbesondere Nichtwissensfragen und verschiedene begünstigende Faktoren dazu geführt haben, dass die US-amerikanische Umweltorganisation Environmental Defense Fund (EDF) und das multinationale Chemieunternehmen DuPont zu einer Partnerschaft zusammengefunden haben. ${ }^{104}$ Die

103 Dabei handelt es sich um die Nanotechnology Ordinance der Gemeinde Berkeley (vgl. http://nanotech.lawbc.com/2007/05/city-of-berkeley-issues-manufactured-n anoscale-material-reporting-guidance/ 13.05.2014).

104 Wie Yaziji (2006) in der Untersuchung von Kooperationen ungewöhnlicher Akteure ausführt, können sich im Kontext gewisser Themen- und Interessenkonstel- 
beiden Organisationen haben bereits in vorangegangenen Projekten Kooperationserfahrungen gesammelt, die für die Zusammenarbeit in der Nanotechnologie förderlich gewesen sind. Auch haben beide Organisationen eine Regulierungslücke in der US-amerikanischen Nanotechnologiepolitik festgestellt, mit der sie nachteilige Effekte für die Interessen ihrer eigenen Organisation verbinden. Wie ein Interviewpartner der Umweltorganisation erläutert, hat EDF die Rolle der Regierung in diesen Fragen als allzu zurückhaltend wahrgenommen und beobachtet, dass auch Herstellerfirmen diese Einschätzung teilen. Dies hat seine Organisation in der Idee bestärkt, mittels eines kooperativen Vorgehens, eigene Lenkungsmaßnahmen zu entwickeln:

„Aus der Perspektive von EDF, war es teilweise dadurch motiviert, dass die Regierung sehr wenig unternommen hat. Da war eine Art Vakuum. Und das war für uns frustrierend aber auch für DuPont und einige andere Firmen, welche die Nanotechnologie anwendeten und auf der Suche nach Mechanismen waren, mit denen sie potenzielle Risiken analysieren und gegen außen kommunizieren konnten.“ (Richard Denison, Environmental Defense Fund, 6. März 2008, Übersetzung der Autorin).

Die Umweltorganisation EDF verfolgt mit diesem Vorstoß auch das Ziel, die Aufmerksamkeit der Regierung auf die Problematik zu lenken, dass eine wachsende Zahl neuer Produkte laufend in einem vergleichsweise rechtsfreien Rahmen auf den Markt gelangt. Angesichts des hohen Zeitbedarfs, den die Ausarbeitung und die Inkraftsetzung einer gesetzlichen Regulierung in Anspruch nimmt, ist EDF zum Schluss gelangt, dass dieser Regulierungslücke mit einer selbstregulatorischen Lenkungsmaßnahme begegnet werden muss:

„Während wir in der Lage waren, eine Alternative zur Regierungsarbeit zu entwickeln, zielte ein großer Teil unseres Interessens darauf, die Regierung dazu zu bringen, mehr zu tun. Es war ziemlich klar, dass sich der Prozess, sogar unter den besten Bedingungen ziemlich langsam bewegt und dass in der Zwischenzeit die Firmen bereits Produkte auf den Markt brachten. Da war

lationen Übereinstimmungen der ansonsten meist als antagonistisch wahrgenommenen kommerziellen Interessen von Unternehmen und der ideellen Missionen zivilgesellschaftlicher Organisationen entwickeln. Dabei erweisen sich diese Kooperationen für beide Seiten als nutzenstiftend. Wie Yaziji (2006) weiter beschreibt, liegt eine der zentralen Voraussetzungen einer solchen Kooperation in der produktiven Zusammenarbeit der beiden Antagonisten. Dies hat sich auch in den Interviews gezeigt. 
viel Aktivität gepaart mit sehr wenig Anleitung." (Richard Denison, Environmental Defense Fund, 6. März 2008, Übersetzung der Autorin).

Der Vertreter von DuPont teilt die Einschätzung, dass in der Zeit, in welcher die Behörden entscheiden, ob eine Regulierung benötigt wird und gegebenenfalls eine solche ausarbeiten, selbstregulatorische Schemata, wie das Nano Risk Framework notwendig sind. Somit können trotz der unklaren Regulierungssituation sichere Produkte auf den Markt gebracht werden:

„Während die Regierungsstellen mit dem Überprüfen beschäftigt sind und damit, zu entscheiden, welche Regulierung sie ergänzen oder verändern müssen, können Produkte unter Berücksichtigung solcher Selbstverantwortungsschemen entwickelt werden." (Nanotechnologieverantwortlicher, DuPont \& American Chemical Council (ACC), 22. Januar 2008, Übersetzung der Autorin).

Derselbe Interviewpartner von DuPont stellt das Schema als eine zentrale Strategie im Umgang mit Nichtwissensfragen dar, die bezüglich der Sicherheit von Nanomaterialien auftauchen:

„Wir wollen nicht generalisieren und sagen, dass Nanomaterialien toxisch sind. Das kann man nicht sagen, man muss darüber sprechen, was wir über das Gefahrenprofil dieses Materials wissen. Und so liegt die Idee darin, mittels eines systematischen Ansatzes die Möglichkeit zu schaffen, dass solche Ungewissheiten auf dem Weg zur Kommerzialisierung angesprochen werden können." (Nanotechnologieverantwortlicher, DuPont \& American Chemical Council (ACC), 22. Januar 2008, Übersetzung der Autorin).

Er erläutert weiter, dass seine persönliche Strategie für den Umgang mit Nichtwissensfragen weniger bei Gefahrenabschätzungen liegt, die er als spekulativ empfindet. Vielmehr steht für ihn die Entwicklung eines systematischen und schrittweisen Vorgehens im Zentrum. Dieses ermöglicht ihm spezifische Fragen, wie solche nach der Exposition und der Gefahrenminimierung zu beantworten:

„Tja, als allererstes sollte man nicht die Gefahr abschätzen. Man benötigt einen Prozess, der die Einschätzung ermöglicht, ob es eine Gefahr gibt oder nicht. So präjudiziert das Schema nicht a priori, dass etwas ein gefährliches Risiko ist, es sagt, dass es hier einen systematischen Prozess gibt, um die Frage zu beantworten und dass hier die Schritte sind, die man ergreifen kann, um eine verantwortungsvolle Entwicklung sicherzustellen." (Nanotechnologieverantwortlicher, DuPont \& American Chemical Council (ACC), 22. Januar 2008, Übersetzung der Autorin).

Unabhängig der konkreten Ausgestaltung eines solchen Programms, muss seine Passung auf die spezifischen Eigenschaften der Materialien und ihre 
Anwendungen sichergestellt werden. Das Ziel insbesondere des Verantwortlichen von DuPont liegt darin, eine den identifizierten Risiken angemessene Regulierung zu entwickeln, also sowohl eine Unter- als auch eine Überregulierung zu vermeiden:

„Es ist wirklich wichtig sicherzustellen, dass was immer wir tun, ob es ein Schema ist oder ob es Forschungsprotokolle sind, ob es ein Grenzwert ist oder ob es Regulierungen sind; dass diese zum Material und zu seiner Anwendung passen und dass die Vorschriften angesichts der identifizierten Risiken angemessen sind. Jegliche Unausgewogenheit ist unerwünscht; sei es eine Überregulierung oder eine Unterregulierung." (Nanotechnologieverantwortlicher, DuPont \& American Chemical Council (ACC), 22. Januar 2008, Übersetzung der Autorin).

Das gemeinsame Entwickeln eines Schemas bedingt jedoch auch Kompromissbereitschaft und Entgegenkommen auf beiden Seiten. So haben beide Seiten in verschiedenen Bereichen Abstriche machen müssen, wie der Vertreter von EDF erläutert:

„Wir haben konzeptuell beschrieben, wie Sicherheit erreicht werden kann, auch wenn die Verantwortung dafür zwischen verschiedenen Akteuren der Lieferkette aufgeteilt ist. Es ist eine Herausforderung, das Schema zu implementieren. In einer idealen Welt mit unlimitierten Ressourcen könnte man sicherlich einen besseren Ansatz finden. Es gibt verschiedene Beispiele, wo wir gerne einen aggressiveren Ansatz verfolgt hätten und DuPont vielleicht einen weniger aggressiven Ansatz." (Richard Denison, Environmental Defense Fund, 6. März 2008, Übersetzung der Autorin).

Die Kooperation von EDF und DuPont hat im US-amerikanischen Regulierungsdiskurs Aufmerksamkeit hervorgerufen. Verschiedene Interviewpartner reflektieren über Vor- und Nachteile und ihre persönliche Einstellung zu selbstregulatorischen Ansätzen. Während das Nano Risk Framework inhaltlich häufig gelobt wird, wird die grundsätzliche Wirksamkeit selbstregulatorischer Ansätze unterschiedlich bewertet. Eine Interviewpartnerin der Rice Universität, die in einem Anspruchsgruppennetzwerk mit EDF und DuPont zusammenarbeitet, bewertet das Programm als willkommenen Schritt in eine lobenswerte Richtung:

„Eine Partnerschaft zwischen der Industrie und einer Umweltorganisation ist sehr lobenswert und ich fühle mich dadurch ermutigt. Dennoch, das Schema befindet sich in einem frühen Stadium, und ob es von anderen Firmen übernommen wird, oder nicht und ob es zu einem Mittel für die übrige Fachgemeinschaft wird, um gute Informationen über Nanomaterialienrisiken zu bekommen, muss sich erst zeigen." (Leitungsmitglied, CBEN, Rice University, 21. Januar 2008, Übersetzung der Autorin). 
Der Vorteil von freiwilligen Maßnahmen wird darin gesehen, dass sie im Gegensatz zur gesetzlichen Regulierung rasch und einfach umgesetzt werden können. Wie dieselbe Wissenschaftlerin erläutert, kann damit das Augenmerk rasch auf dringende Themen gelenkt werden. Auch lassen sich Herstellerfirmen so dazu ermuntern, rechtzeitig Risikomanagementmaßnahmen im Bereich der Nanomaterialien zu ergreifen:

„Neue Gesetze durch das System durchzubringen, ist ein extrem zeitraubender Prozess. Und deshalb sind diese freiwilligen Programme ein Weg, Aufmerksamkeit auf die dringendsten Fragen zu lenken und mit der Industrie im Gespräch darüber zu bleiben, dass es wichtig ist, über all diese Dinge nachzudenken. Bevor fünf oder zehn Jahre vergangen sind und wir immer noch versuchen, die Gesetze zu schaffen. Deshalb denke ich, dass diese freiwilligen Programme, wie das Schema von Environmental Defense Fund und DuPont oder das Datenmeldeprogramm der EPA und die Grenzwertsetzungsaktivitäten alle kollektiv positive Entwicklungen darstellen." (Leitungsmitglied, CBEN, Rice University, 21. Januar 2008, Übersetzung der Autorin).

Selbstregulatorische Steuerungs- und Lenkungsmaßnahmen von regierungsexternen Akteuren werden zwar als effektive Maßnahmen beschrieben, gelten jedoch nicht als tauglicher Ersatz einer gesetzlichen Regulierung. Der Interviewpartner des Wilson-Zentrums bewertet die Selbstregulierung als eine Facette von unterschiedlichen ,sozialen Aufsichtsmechanismen', womit regierungsexterne Akteure Regierungsfunktionen übernehmen. Angesichts des Defizits in der US-amerikanischen Nanotechnologieregulierung, beschreibt er diese sozialen Aufsichtsmechanismen als einen effektiven Ansatz, den die Hersteller nicht ignorieren können:

„Unsere Arbeit ist fast eine Regierungsfunktion. Das bezeichne ich als ,sozialen Aufsichtsmechanismus'. Manchmal scheint dieser möglicherweise effektiver zu funktionieren als die aktuelle Regierungsaufsicht. Diese Kombination an genauer Überprüfung durch die Presse, durch Nichtregierungsorganisationen und durch Gruppen wie die unsere, bietet eine Art soziale Kontrolle, die Firmen nicht wirklich ignorieren können." (Leitungsmitglied, Woodrow Wilson International Center for Scholars, 4. März 2008, Übersetzung der Autorin).

Der Interviewpartner zeigt sich jedoch überzeugt, dass dieser soziale Kontrollmechanismus keine Regierungsaktivitäten ersetzen kann, da Stiftungsgelder im Gegensatz zu öffentlichen Mitteln immer nur kurzfristig und zielorientiert fließen:

„Stiftungen gewähren keine langfristigen Mittel, wie man es von der Regierung her kennt, die eine langfristigere Verpflichtung eingeht, oder die ein Amt einrichtet, welches diese Art von Funktion auf einer etwas nachhaltigeren Ba- 
sis wahrnimmt.“ (Leitungsmitglied, Woodrow Wilson International Center for Scholars, 4. März 2008, Übersetzung der Autorin).

Unmittelbar nach seinem Erscheinen ist das Schema teilweise heftig kritisiert worden. Eine Kooperation, die sich als Arbeitskoalition (Labor Coalition) bezeichnet und die Umweltorganisationen wie Greenpeace, ETC und Friends of the Earth aber auch Gewerkschaften umfasst, kritisiert das NRF in einem offenen Brief an die internationale Nanotechnologiegemeinschaft als grundsätzlich falschen Ansatz. Angesichts der drängenden Probleme, wie die ungelösten Umwelt-, Gesundheits- und Sicherheitsfragen, bezeichnen die Autorinnen und Autoren freiwillige Maßnahmen als Hemmnis einer dringend benötigten gesetzlichen Regulierung:

„Wir weisen die vorgeschlagene freiwillige Maßnahme als komplett falsch zurück. Freiwillige Regulierungen sind oft dazu verwendet worden, eine strenge gesetzliche Regulierung zu schwächen oder zu verzögern. Es sollte als Taktik angesehen werden, dringend benötigte Regulierung zu verzögern und die Beteiligung der Öffentlichkeit vorwegzunehmen. Die rasche Kommerzialisierung der Nanotechnologie erfordert fokussierte Umwelt-, Gesundheits- und Sicherheitsforschung, die aussagekräftige und offene Diskussion sozialer Implikationen und dringende Aufsichtsmaßnahmen." (Labor Coalition 2007, Übersetzung der Autorin).

Die in den offenen Brief involvierten Organisationen befürchten, dass von der Industrie getragene selbstregulatorische Initiativen dazu führen, dass die Politik ihre Regulierungsverantwortung an diese oder weitere gesellschaftliche Akteure delegiert, statt selbst regulatorisch aktiv zu werden:

„Unglücklicherweise ist die DuPont-EDF Maßnahme bestenfalls eine Werbekampagne, die von dringenden, weltweiten Aufsichtsprioritäten für die Nanotechnologie ablenkt. Schlimmstenfalls kann die Initiative zu einer sorglosen Politik führen. Damit wird ein Präzedenzfall für die Delegation der Regulierungsverantwortung von den traditionell für den Schutz der Gesundheit, Umwelt und Sicherheit zuständigen gesellschaftlichen Institutionen an die Industrie geschaffen.“ (Labor Coalition 2007, Übersetzung der Autorin).

Neben dem EDF-DuPont Schema haben in den Vereinigten Staaten ähnlich wie in Deutschland auch Branchenverbände Steuerungsansätze etabliert (Kapitel 3.6.2). Beispielsweise hat der Amerikanische Chemierat (American Chemistry Council, ACC) ein Nanotechnologie-Gremium (Nanotechnology Panel) eingesetzt. ${ }^{105}$ Wie der Verantwortliche von DuPont

105 Siehe dazu http://nanotechnology.americanchemistry.com/Nanotechnology/What -Is-Nanotechnology (13.05.2014) 
erläutert, hat der ACC auch ein vorsorgeorientiertes Programm (responsible care) eingeführt, das übergreifend angelegt ist und sämtliche unter TSCA regulierten Chemikalien betrifft:

„Es gibt allgemeinere Programme, wie ,responsible care'. Dabei handelt es sich um ein vom amerikanischen Chemierat entwickeltes Chemikalienprogramm, das Chemikalien, die unter TSCA reguliert werden, abdeckt und das von Firmen unterschrieben worden ist." (Nanotechnologieverantwortlicher, DuPont \& American Chemical Council (ACC), 22. Januar 2008, Übersetzung der Autorin).

Zudem hat die wissenschaftliche Fachgesellschaft Amerikanische Chemiegesellschaft (American Chemical Society, ACS) Laborsicherheitsrichtlinien für den Umgang mit Nanomaterialien publiziert. ${ }^{106}$ Diese zielen auf eine strikte Kontrolle oder Vermeidung der Exposition von Arbeitskräften $\mathrm{ab}$, solange nicht mehr Wissen bezüglich Nanomaterialiensicherheit vorhanden ist. Die Richtlinien betreffen insbesondere Aspekte wie die Vermeidung von Hautkontakt und Respiration sowie die Anwendung einer guten Laborpraxis.

\subsubsection{Zusammenfassung Vereinigte Staaten: Ein Regulierungsdefizit und eine regierungsexterne Initiative}

Die in den US-amerikanischen Regulierungsdiskurs involvierten Akteure umfassen verschiedene auch am Abschätzungsdiskurs beteiligte Organisationen. Dazu zählen die Vorsorgebehörden EPA, FDA, OSHA und NIO$\mathrm{SH}$, das Forschungszentrum Center for Biological and Environmental Nanotechnology (CBEN) an der Rice Universität und das Wilson-Zentrum. Zusätzlich engagieren sich die Umweltorganisation Environmental Defense Fund und die chemische Industrie, wie ihre Branchenorganisationen ACC und ACS und das Unternehmen DuPont.

Die behandelten Themen umfassen die grunsätzliche, in der US-amerikanischen Nanotechnologiepolitik erfolgte Verflechtung der Innovationen mit den Implikationen. Ebenfalls thematisiert werden Nanomaterialien und die Frage nach ihrer Behandlung durch die bestehende Chemikalien-, Arbeitssicherheits-, Kosmetik-, Lebens- und Arzneimittelgesetzgebung. Dabei wird insbesondere die regulatorische Ungleichbehandlung der Na-

106 Siehe http://www.acs.org/content/acs/en/about/governance/committees/chemicals afety/safetypractices/nanotechnology-safety-resources.html (06.05.2014). 
notechnologie in den unterschiedlich gelagerten Statuten thematisiert. Während Nanomaterialien in Pharmaka, Medizinalprodukten und Pestiziden als wirksam reguliert gelten, werden diese in den bereits intensiv vermarkteten Bereichen Chemikalien, Kosmetika und Lebensmittel als unterreguliert bezeichnet.

Im Regulierungsdiskurs ebenfalls zum Thema wird die das US-amerikanische Umweltrecht prägende Regulierungskultur der, Vermeidungsorientierung' bzw. der ,Wissenschaftsbasierung'. Diese verhindert es, bei der Vermutung potenziell nachteiliger Implikationen regulatorische Maßnahmen zu ergreifen, was unter der europäischen Vorsorgeorientierung möglich ist. Stärker als in Europa erscheint der behördliche Diskurs in den Vereinigten Staaten auch von der Problematisierung der Abwesenheit wissenschaftlich gesicherter Daten und einer ausgeprägten Antagonie zwischen Industrie und Regulierungsbehörden beeinflusst zu sein. Dabei stellen auch US-amerikanische Interviewpartner das neoliberale Paradigma infrage, dass Regulierung innovationshemmend ist. Vielmehr wird die Haltung vertreten, dass eine gesetzliche Regulierung das Nichtwissen reduziert. In dieser Sichtweise führt die Klärung der regulatorischen Behandlung zu Rechtssicherheit und liegt somit auch im Interesse der Industrie.

Als zentrale Praxis kann die in den Vereinigten Staaten erfolgte gesetzliche Behandlung der Nanotechnologie in einem koordinierten Regelwerk bezeichnet werden. Dabei ist die Nanotechnologie-Forschung und Entwicklung im eigens dafür geschaffenen Nanotechnologie Forschungs- und Entwicklungsgesetz (NRDA) verankert worden. Dieses Gesetzt betrifft auch Risiken und potenzielle nachteilige Implikationen. Dabei propagiert es einen inkrementellen Ansatz, indem es deren gesetzliche Behandlung in die existierende Gesetzgebung integriert.

Damit verfolgt die US-amerikanische Regierung die Praxis der strukturellen Verflechtung der Förderung der Technikentwicklung mit der Vermeidung ihrer potenziellen nachteiligen Umwelt-, Sicherheits-, Gesundheits- und sozialen Implikationen, was verschiedentlich kritisiert wird. Damit verbunden lässt sich nicht nur im Abschätzungsdiskurs, sondern auch bei der Regulierung eine Priorisierung der Innovationen und eine Marginalisierung der Implikationen beobachtet. Diese Verflechtung und Ungleichbehandlung wird von verschiedenen Interviewpartnern als eine der zentralen Herausforderungen der Nanotechnologieregulierung in den Vereinigten Staaten gesehen. Diese verbreitet als unbefriedigend bezeichnete Situation wird auch auf die Regulierungsunwilligkeit der in den Jahren 
2001-2009 regierenden republikanischen Administration unter George W. Bush und die damit verbundene chronische Unterfinanzierung der Vorsorgebehörden zurückgeführt.

Im Umgang mit dem problematisierten Mangel an wissenschaftlich gesicherten Daten zur Nanotechnologie entwickelt sich ein Dispositiv zum Regulieren. Da dies aufgrund der fehlenden Daten gesetzlich nicht möglich ist, werden freiwillige oder selbstregulatorische Ansätze ergriffen. Dabei führen die Vorsorgebehörden selbst wissenschaftliche Studien durch oder geben solche in Auftrag und die EPA hat ein freiwilliges Datenmeldeverfahren etabliert, in welchem sie die Industrie auffordert, ihr die von ihr erhobenen Sicherheitsdaten zu Nanomaterialien mitzuteilen. Dieses Meldesystem wird jedoch als zu spät erfolgend und durch seine Freiwilligkeit als zu wenig weitgehend kritisiert. Die von verschiedenen Interviewpartnern als bescheiden bezeichnete Meldequote von 123 Substanzen scheint diese Kritiken zu bestätigen. Die Kritik wird insbesondere auch durch das Nanotechnologiekonsumprodukteinventar des Wilson Zentrums untermauert, das in diesem Zeitraum von 743 sich auf dem Markt befindenden Nanotechnologieprodukten ausgeht. Die mit dem NMSP verknüpften Ziele, wie das Schaffen von mehr Klarheit und die vom EPAVerantwortlichen im Interview genannte Idee, dass sich das NMSP bei zu wenig Meldungen in ein obligatorisches Schema transferieren lasse, sind allesamt nicht erreicht worden. Vereinzelte Stoffe in ausgewählten Anwendungen, wie Nanosilber in Waschmaschinen sind im Rahmen bestehender Gesetze reguliert worden. Die verbreitete Wahrnehmung einer Regulierungslücke hat in den Vereinigten Staaten zur Praxis geführt, dass einzelne Bundesstaaten und Gemeinden die Nanotechnologie mit lokalen Verordnungen reguliert haben.

Das grundsätzliche Defizit in der regulatorischen Behandlung der Nanotechnologie durch die nationalen politischen Institutionen hat zu einem Dispositiv zur Übernahme von sozialen Aufsichtsmechanismen und zur Entwicklung selbstregulatorischer Ansätze durch regierungsexterne Akteure geführt. Dabei ist insbesondere die Kooperation der Umweltorganisation EDF mit dem multinationalen Chemikalienhersteller DuPont bedeutsam. Die beiden Organisationen haben ein Risikomanagementverfahren entwickelt, das im Regulierungsdiskurs Beachtung findet. Allerdings hat sich ihr Ziel, dass sich das System zu einem branchenweiten Standard entwickelt, bis zum Abschluss dieser Studie nur insofern erfüllt, als dass die ISO Auszüge daraus in einem technischen Bericht veröffentlicht hat. Darin zeigen sich die vielfältigen Schwierigkeiten, die sich durch die ge- 
sellschaftliche Distribution von Regulierung an regierungsexterne Instanzen und selbsternannte Anspruchsgruppen ergibt, wie ihre fehlende Durchsetzbarkeit, Verbindlichkeit und ihr mangelnder Beitrag zur Rechtssicherheit.

\subsection{Europäische Union: REACH und ein Forschungskodex}

Die zentrale Thematik des europäischen Regulierungsdiskurses fokussiert auf die Frage nach der Behandlung der Nanomaterialien unter der bestehenden Gesetzgebung. ${ }^{107}$ Dabei steht die Kontroverse zwischen der Kommission und dem Parlament um die unterschiedliche Einschätzung der $\mathrm{Zu}$ ständigkeit der Chemikaliengesetzgebung REACH für die Regulierung von Nanomaterialien im Zentrum. Daneben sind auch nichtverbindliche Rechtsakte der Kommission, wie der Verhaltenskodex für ethische Nanotechnologieforschung bedeutsam sowie die im sektoralen Produktrecht punktuell ergriffenen gesetzlichen Maßnahmen. Das Kapitel zeigt das grundsätzlich netzwerkförmige Regulierungsverständnis auf Unionsebene auf, das nicht nur Gesetze, sondern auch informelle Maßnahmen vorsieht. Weiter zeigt sich auf der Unionsebene ein integrativer Regulierungsansatz. Hier wird stärker als in den anderen untersuchten Ländern versucht, die Nanotechnologie in das bestehende gesetzliche Rahmenwerk einzugliedern. Wie das Kapitel weiter zeigt, liegt eine der zentralen Herausforderungen der europäischen Nanotechnologieregulierung in der Umsetzung des im europäischen Umweltrecht zentral verankerten Vorsorgeprinzips.

\subsubsection{Nanomaterialien und REACH}

In der Europäischen Kommission sind insbesondere die auch im Abschätzungsdiskurs bedeutsamen Generaldirektionen (DG) Umwelt (ENV), For-

107 Diese wird auf Unionsebene durch die vier Institutionen Rat, Kommission, Parlament und Gerichtshof getragen. Während der Rat die übergeordneten Ziele definierte, entwirft die Kommission die Gesetze und überwacht deren Implementierung. Demgegenüber verfasst das Parlament die Detailformulierung und entscheidet über die formelle Einordnung (Gesetz oder Verordnung). Der Gerichtshof stellt sicher, dass das Recht der Europäischen Union mit den Inhalten der Verträge übereinstimmt (vgl. z.B. McCormick 2001). 
schung \& Innovation (RTD), Unternehmen \& Industrie (ENTR) und Gesundheit \& Verbraucher (Sanco) in den Regulierungsdiskurs um die Nanotechnologie involviert. Die Zuständigkeiten für übergreifende Aspekte werden unter den Generaldirektionen aufgeteilt, wie ein Interviewpartner der Kommission ausführt:

„Nanotechnologie wird von unterschiedlichen DGs berührt. Die wichtigsten sind Enterprise und Umwelt, Sanco und Forschung. Aber es gibt wahrscheinlich auch noch andere DGs, die etwas mit Nanotechnologie zu tun haben. Es ist eine Kompetenzaufteilung." (Nanotechnologieverantwortlicher, Europäische Kommission, DG Research, 7. Oktober 2008).

Bei der Analyse des Regulierungsdiskurses auf Unionsebene fällt auf, dass das Vorsorgeprinzip häufig angesprochen wird und dass das Nichtwissen im Bereich der Nanotechnologie als hoch eingestuft wird. So erläutert ein Vertreter der Generaldirektion Forschung, dass eine wichtige Strategie der Kommission im Umgang mit dem mit potenziellen nachteiligen Implikationen der Nanotechnologie verbundenen Nichtwissen darin besteht, das Vorsorgeprinzip anzuwenden:

„Wir glauben, dass in bestimmten Bereichen die Risikomethodologie nicht vollständig ist und die Risiken nicht völlig identifizierbar sind und deswegen die Anwendung des Vorsorgeprinzips relevant ist." (Nanotechnologieverantwortlicher, Europäische Kommission, DG Research, 7. Oktober 2008).

Ein zentraler Aspekt des Regulierungsdiskurses der Nanotechnologie betrifft die Frage nach der Behandlung von Nanomaterialien unter der REACH-Verordnung (siehe Kapitel 2.4.3). In ihrer Mitteilung zu Regulierungsaspekten der Nanotechnologie aus dem Jahr 2008, die eine Rechtsanalyse der bestehenden Gesetzgebung umfasst, gelangt die Kommission zum Ergebnis, dass der bestehende Rechtsrahmen unter der Chemikaliengesetzgebung REACH die potenziellen Umwelt-, Gesundheits- und Sicherheitsimplikationen der Nanotechnologie weitgehend abdeckt (COM 2008b). Diese Einschätzung stellt das Parlament in einer Erklärung im Jahr 2009 grundsätzlich infrage und fordert den spezifischen Eigenschaften synthetisch hergestellter Nanomaterialien angepasste gesetzliche Regulierungsschritte (P6_TA 2009) (vgl. dazu auch Kapitel 2.4.3). Dazu kritisiert ein Abgeordneter des Europäischen Parlaments die Einschätzung der Kommission, dass der bestehende Rechtsrahmen für die Nanotechnologie ausreicht, als nicht zutreffend. Seiner Ansicht nach fehlen Strategien im Umgang mit dem mit der Sicherheit von Nanomaterialien verbundenen Nichtwissen: 
„Daher empfinden wir die Mitteilung der Kommission als Schönfärberei. Hier wird das geltende Recht sehr allgemein und theoretisch auf Nano angewendet. Es wird zwar zugestanden, dass die Methoden ergänzt werden müssten. In dieser Mitteilung fehlt uns aber die Schlussfolgerung, dass wenn die Methoden nicht greifen und man keine Aussagen über die Sicherheit treffen kann, man auch nicht sagen kann, dass es durch das Gesetz abgedeckt sei.“ (Nanotechnologiespezialist und Abgeordneter, Europäisches Parlament, 30.10.2008).

Die federführend in die Verfassung der Rechtsanalyse involvierte Vertreterin der Generaldirektion Umwelt zeigt sich irritiert über die Kritik an der Rechtsanalyse. Sie erläutert, dass die Kommission feststellt, dass das Gesetz nicht implementiert werden kann, weil das Wissen über wirksame Testrichtlinien fehlt. Dies ist ihrer Ansicht nach eine brisante Aussage:

„Wir haben die Gesetze analysiert und gesehen, dass Nanomaterialien prinzipiell abgedeckt sind; das ist die eine Sache. Aber andererseits sagen wir, dass wir dieses Gesetz nicht implementieren können, weil wir das Wissen über die Testrichtlinien nicht haben und das ist eine ziemlich starke Aussage. Ich bin überrascht, dass jemand sagt, dass wir mit unserer Analyse nicht weit genug gehen." (Nanotechnologieverantwortliche, Europäische Kommission, DG Umwelt, 13. November 2008, Übersetzung der Autorin).

Die Kommission sieht die zentrale Herausforderung der Nanotechnologie in den umfangreichen Wissenslücken und bei der Unklarheit, wie die Nanotechnologie unter der bestehenden Gesetzgebung implementiert werden kann. Demgegenüber sieht das Parlament das größte Problem darin, dass der Rechtsrahmen fehlt. Dazu führt dieselbe Vertreterin der Kommission weiter aus, dass die Kommission nicht die Haltung vertritt, dass nichts getan werden soll. Vielmehr problematisiert die Rechtsanalyse die Nichtimplementierbarkeit der Regulierung. Deshalb hält die Interviewpartnerin es für falsch, den Fokus auf die Entwicklung neuer Gesetze zu legen, die wiederum nicht implementierbar sind. Vielmehr soll die Implementierung der bestehenden Regulierung vorangetrieben werden:

„Das soll sicher nicht bedeuten, dass wir nichts tun sollen. Im Gegenteil, die Schlussfolgerung in der Mitteilung sagt, dass es ein großes Problem ist, dass wir die Regulierung nicht implementieren können. Aber die Arbeit soll sich darauf konzentrieren und nicht auf den Versuch, neue regulatorische Ansätze zu entwickeln, die mit den genau gleichen Problemen konfrontiert werden, nämlich, dass sie nicht implementiert werden können." (Nanotechnologieverantwortliche, Europäische Kommission, DG Umwelt, 13. November 2008, Übersetzung der Autorin).

Ein Vertreter der Generaldirektion Unternehmen stellt fest, dass sich die Kommission und das Parlament darin einig sind, dass sie mehr Testmetho- 
den und mehr Sicherheitsdaten für die Risikoanalysen benötigen. Nicht einig sind sie sich in der Frage, ob dies auf eine Regulierungslücke hindeutet oder ob es in den Aufgabenbereich der Implementierung fällt:

„Wo wir uns einig sind, ist darin, dass wir mehr Testmethoden benötigen. Wir brauchen mehr Daten. Wir benötigen mehr Risikoabschätzungsmodelle. Wir sind uns alle darin einig, dass wir das Vorsorgeprinzip für die Risikoabschätzung und das Risikomanagement benötigen. Wo wir uns nicht einig sind, ist in der Frage, wie wir diese Maßnahmen bezeichnen. Wir sagen, dass dies keine Regulierungslücke ist, weil wir eine Regulierung haben. Wir bezeichnen es als Implementierung der Regulierung." (Nanotechnologieverantwortlicher, Europäische Kommission, DG Unternehmen und Industrie, 3. Dezember 2008, Übersetzung der Autorin).

Die innerhalb der Institutionen der Europäischen Union vertretenen divergierenden Auffassungen zur regulatorischen Behandlung von Nanomaterialien dominieren den Regulierungsdiskurs auch in den nachfolgenden Jahren. Selbst im Jahr 2013 scheinen sich die Haltungen nicht angenähert zu haben, wie zwei in Nature Nanotechnology veröffentlichte Artikel zeigen. Im einem Artikel identifiziert ein Nanomaterielienspezialist der Technischen Universität Dänemark das zentrale Defizit der REACH-Verordnung in deren genuinen Logik, chemische Substanzen nach ihrer Identität statt nach ihren Eigenschaften zu regulieren (Foss Hansen 2013). Dies führt dazu, dass chemisch identische Substanzen mit unterschiedlichen Partikelgrößen und somit anderen Oberflächeneigenschaften, gesetzlich gleich behandelt werden. Dabei werden die sich teilweise von der Makroform grundlegend unterscheidenden Eigenschaften nanoskaliger Substanzen gesetzlich nicht berücksichtigt. Angesichts der hohen Anzahl der sich auf dem europäischen Markt befindenden Nanomaterialien, beurteilt der Autor dies als besorgniserregend (Foss Hansen 2013). ${ }^{108}$ Diese Einschätzung zeigt sich auch in den Interviews: Hier sieht die Interviewpartnerin der Generaldirektion Umwelt die größte regulatorische Herausforderung in der genuinen Logik der Nanoskaligkeit, in welcher sich chemische und physikalische Eigenschaften von Stoffen nicht strukturabhängig, sondern größen- und formabhängig verändern. Sie spricht diesbezüglich von einem ,wissenschaftlichen Paradigmenwechsel', in welchem weder die molekulare Form eines Stoffes, noch darauf zurückführbare strukturelle Eigenschaften Rückschlüsse auf seine Toxizität ermöglichen:

108 Im Jahr 2012 befinden sich mehr als sechzig unterschiedliche Nanomaterialien auf dem europäischen Markt (COM 2012a). 
„Mit Nanomaterialien erfolgt ein wissenschaftlicher Paradigmenwechsel. Früher gab es Chemikalien mit einer einzigartigen molekularen Formel und einer spezifischen Gruppe Eigenschaften, die über ihre toxischen und ökotoxischen Effekte entschieden. Aber mit Nano gibt es größen- und formabhängige Eigenschaften. Man hat die gleichen chemischen Substanzen mit der gleichen chemischen Formel. Abhängig von der Größe sind ihre Eigenschaften unterschiedlich. Dies ist neu in der gesamten Gesetzgebung." (Nanotechnologieverantwortliche, Europäische Kommission, DG Umwelt, 13. November 2008, Übersetzung der Autorin).

Demgegenüber erläutert ein Vertreter der Generaldirektion Unternehmen und Wirtschaft in einem zweiten in der gleichen Ausgabe von Nature Nanotechnologie publizierten Artikel die Einschätzung, dass sich Nanomaterialien in ihrem Gefährdungspotenzial nicht von herkömmlichen Chemikalien unterscheiden (Tajani 2013). Angesichts der hohen Unsicherheit, die hinsichtlich des Zusammenhangs zwischen den Eigenschaften und dem Verhalten von verschiedenen Nanomaterialien besteht, soll nicht auf der gesetzlichen Ebene angesetzt werden. Vielmehr sollen die Anhänge von REACH modifiziert werden. Dabei argumentiert der Autor insbesondere mit dem Gleichbehandlungsprinzip:

„Hier fehlt das Argument, warum Nanomaterialien in diesem Zusammenhang anders als andere Chemikalien behandelt werden sollten. Insbesondere gibt es keinen Hinweis darauf, dass Nanomaterialien als eine Substanzkategorie gefährlicher sind als andere chemische Substanzen, oder dass die Risiken von gefährlichen Nanomaterialien schlimmer sind als diejenigen von konventionellen gefährlichen Chemikalien. Deshalb ist es unklar, weshalb die REACHRegulierung für Nanomaterialien, im Unterschied zu anderen Chemikalien geringere oder gar keine Massenbegrenzungen anwenden sollte." (Tajani 2013, Übersetzung der Autorin).

Im Jahr 2012 veröffentlicht die Kommission eine weitere Regulierungsanalyse (siehe Kapitel 2.4.3). Darin vertritt sie erneut die Haltung, dass Nanomaterialien unter der bestehenden Regulierung ausreichend reguliert sind. Anpassungsbedarf sieht sie lediglich bei den Anhängen von REACH (COM 2012a). Diese Folgerung wird ebenfalls kontrovers diskutiert. Die Kontroverse dreht sich insbesondere um Fragen wie, ob sich traditionelle Ansatze für die Risikobeurteilung und die als notwendig eingeschätzte Einzelprüfung der möglichen Risiken von Nanomaterialien eignen. Weiter wird das Fehlen spezifischer standardisierter Testverfahren und dazu benötigte Geräte diskutiert (SCENIHR 2009; SCENIHR, et al. 2009). Ebenso wird kritisiert, dass von den Herstellern nach wie vor keine detaillierte physikalisch-chemische Charakterisierungsdaten von nanoskaligen Sub- 
stanzen verlangt werden, obwohl Expertenkommissionen dies empfehlen (Aitken, et al. 2011; Hankin, et al. 2011). ${ }^{109}$

Foss Hansen (2013) bewertet die publizierten Informationsanforderungen für nanoskalige Substanzen, die Datenmeldung und die Kombination von Sicherheits- und Expositionsdaten unter REACH als ungenügend. ${ }^{110}$ Zudem hinterfragt er erneut den von der Kommission gezogenen Analogieschluss zwischen Nanomaterialien und herkömmlichen Chemikalien bezüglich der Vorhersehbarkeit ihrer toxischen Wirkung (COM 2012b). Eine vergleichbare Kritik wird auch von der Europäischen Umwelagentur (European Environmental Agency, EEA) angebracht. Beide Kritiken weisen auf verschiedene Fälle von industriell genutzten Chemikalien im 20. Jahrhundert hin, die sich nach ihrer Markteinführung als Verursacher gravierender Gesundheits- und Umweltschädigungen entpuppt haben, wie Aspest und Fluorchlorkohlenwasserstoffe (FCKW) (EEA 2002; EEA 2013; Foss Hansen 2013). Nach Ansicht des Parlamentsabgeordneten überlässt ihre unspezifische gesetzliche Behandlung der Industrie einen umfassenden Spielraum im Umgang mit nanoskaligen Substanzen:

„Das Problem ist, dass es noch keine klaren nanospezifischen Richtlinien gibt. Das heißt, es ist an der Industrie zu entscheiden, wie sie die Nanomaterialien behandelt." (Nanotechnologiespezialist und Abgeordneter, Europäisches Parlament, 30.10.2008).

Zentral für den europäischen Regulierungsdiskurs ist also die Frage nach dem Umgang mit dem hohen Maß an Nichtwissen von synthetischen Nanomaterialien. Dabei werden in den Interviews insbesondere vier Bereiche genannt, in denen sich das Nichtwissen manifestiert:

1. Definition von Nanomaterialien

2. Entwicklung von Testmethoden

3. Behandlung von Nanomaterialien unter REACH

4. Nachweise von konkreten Umwelt- und Gesundheitsrisiken

109 Weitere Kritikpunkte betreffen neben sogenannt passiven Nanostrukturen auch Nanomaterialien zweiter und dritter Generation, die vor der Markteinführung stehen, die jedoch die Europäische Kommission in ihrer zweiten Regulierungsevaluation (COM 2012b) nicht berücksichtigt. Ebenfalls kritisiert wird das Fehlen eines strategischen Konzepts zur Entwicklung von umwelt- und gesundheitsrelevanten Daten und Regulierungen dieses Bereiches (Foss Hansen 2013).

110 Insbesondere als ungenügend bezeichnet Foss Hansen (2013) das inzwischen unter REACH eingeführte Nanomaterialienregister, das im Jahr 2012 lediglich sieben Nanomaterialien enthält (COM-JRC 2012; ECHA 2012; Foss Hansen 2013). 
Hinsichtlich der Definition von Nanomaterialien weist der Interviewpartner aus dem Europäischen Parlament darauf hin, dass sich selbst die Fachleute nicht einig sind, ab wann von einer neuen Substanz bzw. einer neuen Verwendung gesprochen werden kann:

„Die Frage nach dem regulatorischen Umgang mit Nano fängt mit der Frage an, wann etwas nicht mehr das gleiche bzw. anders ist und in welchem Moment neue Informationen eingebracht werden müssen. Darüber können sich nicht einmal die Experten einigen." (Nanotechnologiespezialist und Abgeordneter, Europäisches Parlament, 30.10.2008).

Als ein weiteres Problem identifizieren verschiedene Interviewpartner das hohe Maß an Nichtwissen bezüglich der geeigneten Testmethoden. Wie der Parlamentsabgeordnete erläutert, fehlt im Bereich der Nanotechnologie Wissen in doppelter Hinsicht: Das für eine robuste Risikoeinschätzung notwendige Testwissen fehlt nahezu vollständig. Dadurch bleibt neben dem Gegenstand und dem Inhalt auch die Implementierung von potenziellen Richtlinien unklar. Dies erachtet der Interviewpartner insofern als besorgniserregend, als dass Produkte, die Nanomaterialien enthalten und über deren potenzielle Implikationen wenig Wissen vorhanden ist, in zunehmendem Maße auf den Markt gebracht werden:

„Wir haben das doppelte Problem, dass nicht nur die Vorschriften, sondern auch die nötigen Tests fehlen, um eine korrekte Risikoeinschätzung durchzuführen. Somit ist es unklar, wie diese Vorschriften aussehen sollen. Das ist insofern problematisch, als dass mehr und mehr Nanomaterialien in allen möglichen Erzeugnissen auf den Markt kommen." (Nanotechnologiespezialist und Abgeordnetr, Europäisches Parlament, 30.10.2008).

Die Ansicht, dass die Frage nach den Testmethoden zentral ist, wird auch in der neu geschaffenen Europäischen Chemikalienagentur (ECHA) vertreten. Die Chemikalienagentur wird als die auf Unionsebene zuständige Organisationseinheit für die Behandlung von Chemikalien eingesetzt und gilt als Schlüsselaspekt für die erfolgreiche Implementierung von REACH. Insbesondere arbeitet die Chemikalienagentur eng mit der Kommission, den Mitgliedsstaaten, den Regulierungsbehörden und privaten Firmen an der Registrierung und Evaluation von Chemikalien (Selin 2007b, 70). ${ }^{111}$ Eine Vertreterin der ECHA identifiziert das Kernproblem in

111 Die Chemikalienagentur gilt als Hüterin von REACH auf Unionsebene und soll den Einfluss der europäischen Chemikalienregulierung weiter stärken. Sie ist aber auch auf die Kooperation der Mitgliedsstaaten und die privaten Firmen in der Datenlieferung angewiesen. Selin $(2007 b, 70)$ spricht dabei von einer Euro- 
der Frage nach den toxikologischen Tests, die für Nanomaterialien durchgeführt werden müssen:

„Die Kernfrage, die jedermann kennt und die diskutiert wird, lautet: Welche Art und welche Typen von Ökotoxizitäts- und Toxizitätstests müssen für Nanomaterialien durchgeführt werden?" (Nanotechnologieverantwortliche, Europäische Chemikalienagentur (ECHA), 20. Januar 2009, Übersetzung der Autorin).

Hinsichtlich der Frag nach der Zuständigkeit von REACH für Nanomaterialien, weist der Parlamentsabgeordnete darauf hin, dass sich selbst die Experten nicht darüber einigen können, ab wann von einer neuen Substanz beziehungsweise einer neuen Verwendung gesprochen werden kann:

„Die Frage nach dem regulatorischen Umgang mit Nano fängt mit der Frage an, wann etwas nicht mehr das gleiche bzw. anders ist und in welchem Moment neue Informationen eingebracht werden müssen. Darin können sich nicht einmal die Experten einigen." (Nanotechnologiespezialist und Abgeordneter, Europäisches Parlament, 30.10.2008).

Analog zur Regulierungsanalyse der Kommission, geht ein Interviewpartner der Generaldirektion Unternehmen von der Zuständigkeit von REACH auch ohne explizite Erwähnung des Begriffs der Nanomaterialien aus. Ihm stellt sich vielmehr die Frage, wie REACH an die spezifischen Charakteristika von Nanomaterialien angepasst werden kann:

„Es ist komplett falsch zu sagen, dass REACH Nanomaterialien nicht abdeckt. Alle bekannten Nanomaterialien auf dem Markt sind Substanzen im Sinne von REACH. Nun stellt sich jedoch Frage, ob REACH nicht verbessert werden könnte, um Nanomaterialien spezifisch zu behandeln, das ist ein anderes Thema." (Nanotechnologieverantwortlicher, Europäische Kommission, DG Unternehmen und Industrie, 3. Dezember 2008, Übersetzung der Autorin).

Eine weitere Problematik in der Anwendbarkeit von REACH auf Nanomaterialien wird bei der Produktionsvolumengrenze gesehen. Unter REACH sind bislang nur Substanzen meldepflichtig, die in einem Umfang von mehr als einer Tonne pro Jahr hergestellt werden. Verschiedene Interviewpartner äußern ihre Bedenken darüber, dass diese Tonnagegrenze für Nanomaterialien zu hoch angesetzt ist, da diese häufiger in geringeren Volumina produziert werden. Auch die Vertreterin der Chemikalienagentur

päisierung der weltweiten Chemikalienpolitik und weist der ECHA darin eine zentrale Rolle zu. 
bewertet die Frage nach der Tonnagegrenze als eine der wichtigsten regulatorischen Probleme neben der Definition von Nanomaterialien:

„Die Definition des Nanomaterials ist in den internationalen Foren nicht gelöst und es noch nicht abschließend bestimmt worden, welche Menge synthetischer Nanomaterialien unter dem Produktionsvolumen von einer Tonne hergestellt werden." (Nanotechnologieverantwortliche, Europäische Chemikalienagentur (ECHA), 20. Januar 2009, Übersetzung der Autorin).

Selbst im vorsorgeorientierten Regulierungskontext Europas sieht die Interviewpartnerin der Generaldirektion Umwelt ein zentrales Problem darin, dass gesicherte wissenschaftliche Nachweise fehlen. Ohne diese können weder Richtlinien entwickelt, noch die Tonnengrenze evaluiert werden:

„Für mich liegt das Problem im weiteren Vorgehen: Die Testrichtlinien müssen angepasst werden und es muss festgestellt werden, ob die TonnagenSchwellenwerte ausreichen. Und dazu benötigen wir mehr wissenschaftliche Grundlagen." (Nanotechnologieverantwortliche, Europäische Kommission, DG Umwelt, 13. November 2008, Übersetzung der Autorin).

In den Interviews werden verschiedene Praktiken im Umgang mit diesem Nichtwissen erwähnt. Dieselbe Interviewpartnerin erläutert, dass ihrer Meinung nach die zentrale Strategie in der Nanomaterialienregulierung darin liegen müsste, neben den gesetzlichen Aspekten, insbesondere Implementierungs- und Umsetzungsansätze zu überarbeiten:

„Ein Punkt liegt darin, die Regulierung auf drei verschiedenen Ebenen klar zu trennen: Eine Ebene ist das Regelwerk, die zweite Ebene ist die Anwendung und die dritte ist die Durchsetzung." (Nanotechnologieverantwortliche, Europäische Kommission, DG Umwelt, 13. November 2008, Übersetzung der Autorin).

Für sie steht deshalb die Implementierungsfrage im Vordergrund des Regulierungsdiskurses. Hier kann in einem ersten Schritt die Überarbeitung von Richtlinien, Testmethoden und Expositionsmessungen erfolgen:

„Auf der Ebene der Anwendung muss sichergestellt werden, dass die neuen Materialien auf Risiken und Sicherheit überprüft werden können. Deshalb müssen wir wahrscheinlich die Richtlinien, die Testmethoden und die Expositionsmessungsmethoden etc. überarbeiten und wir müssen uns an die neue Art der Wissenschaft anpassen, die Nanomaterialien darstellen." (Nanotechnologieverantwortliche, Europäische Kommission, DG Umwelt, 13. November 2008, Übersetzung der Autorin).

Eine in der Rechtsanalyse angekündigte Strategie der Kommission für den Umgang mit Nichtwissen bezüglich der Zuständigkeit von REACH für 
Nanomaterialien, liegt darin, eine für Nanomaterialien zuständige Expertenkommission zu etablieren. Dies geschieht im Rahmen einer Subgruppe der Zuständigen Behörden (Competent Authorities, CA) von REACH. Hier wird also die im Abschätzungsdiskurs auf EU-Ebene beobachtete Strategie, die Nanotechnologie in die bestehenden Gremien zu integrieren, durchbrochen und eine neue, nanospezifische Kommission einberufen. Diese Kommission stellt eine administrative Einheit aus Vertretern sämtlicher Mitgliedsstaaten dar, die für die Begleitung der Implementierung und der Verwaltung der REACH-Richtlinie zuständig sind:

„REACH wird unterstützt durch regelmäßige Treffen der Zuständigen Behörden ,Competent Authorities', wie wir sie nennen. Das bedeutet, dass jedes Mitgliedsland eine behördliche Einheit hat, die für die Umsetzung und Verwaltung von REACH in ihrer eigenen Rechtssprechung zuständig ist. Die für REACH zuständigen Behörden haben verschiedene Untergruppen. Die letzte, die gerade im Jahr 2008 geschaffen worden ist, behandelt Nanomaterialien." (Nanotechnologiespezialist, Europäische Kommission, DG Umwelt, 30.10.2008, Übersetzung der Autorin).

Eine weitere Strategie im Umgang von dem mit Nanomaterialien verbundenen Nichtwissen wird in der internationalen Kooperation gesehen. Dazu führt die Interviewpartnerin der ECHA aus, dass der internationalen Kooperation in ihrem Amt strategisch zentrale Bedeutung zukommt. Diese Kooperationen betreffen nicht nur solche mit der Europäischen Kommission und weiteren nationalen Ämtern, wie der US-amerikanischen Umweltbehörden EPA, sondern ebenso solche in internationalen Gremien wie der OECD und der internationalen Standartorganisation ISO. Hier können die Wissenslücken in der Sicherheitsforschung von Nanomaterialien in einer internationalen Zusammenarbeit angegangen werden:

„Für uns ist die Zusammenarbeit mit der Kommission, mit der OECD und mit der ISO und via OECD auch mit der US-amerikanischen EPA und ihren Partnerämtern zentral. Wir denken, dass die Lücken dadurch begründet sind, dass die Wissenschaft hinterherhinkt. Denn was wissenschaftlich untersucht ist, können wir auf REACH anwenden. Aber hoffentlich werden diese Lücken in der nahen Zukunft geschlossen." (Nanotechnologieverantwortliche, Europäische Unionropean Chemicals Agency (ECHA), 20. Januar 2009, Übersetzung der Autorin).

Die Strategie der Europäischen Kommission im Umgang mit dem mit Nanomaterialien verbundenen Nichtwissen liegt also in ihrer Integration in das bestehende Regelwerk. Zudem ist die Identifikation ihrer spezifischen Eigenschaften wichtig. Gleichzeitig treibt die Kommission die Risikoforschung und die Entwicklung geeigneter Testmethoden voran. Weitere 
Strategien umfassen die Kooperation in internationalen Gremien, die Einberufung einer in diesem Fall neuen und nanomaterialienspezifischen Expertengruppe aus den zuständigen Länderbehörden (CA) und darauf aufbauend die Implementierung und Anpassung der gesetzlichen Grundlagen. Zudem lässt sich ein Ringen zwischen der Kommission und dem Parlament um die Anwendung des Vorsorgeprinzips feststellen. Wie die Interviews zeigen, werden auf Unionsebene neben der gesetzlichen Regulierung auch weniger bindende Steuerungs- und Lenkungsansätze diskutiert und eingesetzt. Solche Maßnahmen, sind Gegenstand des nachfolgenden Kapitels.

\subsubsection{Freiwillige Maßnahmen: Empfehlungen der Kommission zu Forschung und Definition}

Im letzten Kapitel des ersten Implementierungsberichts (COM 2007a) zur Integration der Europäischen Nanotechnologiestrategie weist die Kommission auf die Notwendigkeit, einer sicheren und verantwortungsvollen Nanotechnologieentwicklung hin. Auch kündigt sie dazu die Publikation eines Verhaltenskodexes an (Kapitel 2.4.2). Der Kommission fehlt die EUrechtliche Zuständigkeit für den Bereich der Ethik. Deshalb ist für die Regelung der Verantwortlichkeit nur ein rechtlich nicht verbindlicher Akt, wie eine Kommissionsempfehlung möglich (vgl. Eisenberger, et al. 2010). Dazu hat die Europäische Kommission im Jahr 2008 den ersten nanotechnologiespezifischen Rechtsakt ${ }^{112}$ in Form einer rechtlich nicht verbindlichen Empfehlung zu einem Verhaltenskodex für eine verantwortungsvolle Forschung in der Nanotechnologie und in den Nanowissenschaften erlassen. Der Verhaltenskodex ist von der Generaldirektion Forschung entwickelt worden und legt Prinzipien und Leitlinien für die Mitgliedsstaaten und die betroffenen Organisationen und Akteure fest (COM 2008a). Ein in die Verfassung des Verhaltenskodexes involvierter Vertreter der Generaldirektion Forschung erläutert, dass eines der im Aktionsplan beschriebenen Vorhaben darauf abzielt, Verhaltensregeln festzulegen. Als ein internationaler Dialog dazu gescheitert ist, hat die Kommission die Initiative ergriffen und einen eigenen Ansatz entwickelt:

112 Für diesen Rechtsakt gilt aufgrund der fehlenden unionsrechtlichen Zuständigkeit nur die Form einer rechtlich nicht verbindlichen Empfehlung als möglich (vgl. Eisenberger, et al. 2010). 
„Im Aktionsplan wurde angekündigt, dass die Kommission international an einer Entwicklung eines Codes of Conduct mitarbeiten wird. Dazu gab es einen Dialog mit unterschiedlichen Ländern, der jedoch keine Fortschritte zeigte. Daraufhin hat die Kommission beschlossen, selbst die Verantwortung für diesen Bereich zu übernehmen." (Nanotechnologieverantwortlicher, Europäische Kommission, DG Research, 7. Oktober 2008).

Eine Verantwortliche der Generaldirektion Umwelt erläutert den Fokusbereich des Verhaltenskodexes. Während die gesetzliche Regulierung einen klar eingrenzbaren und definierbaren Anwendungsbereich voraussetzt, lassen sich rechtlich nicht verbindliche Ansätze allgemeiner und unspezifischer formulieren. Diese lassen sich auch auf nicht klar eingrenzbare und in der Zukunft liegende Bereiche wie die zukünftige Forschung anwenden:

„Der freiwillige Verhaltenskodex betrifft die Forschung, die in der Zukunft stattfinden wird. Was sich heute hier in der Produktion und auf dem Markt befindet, sollten wir verpflichtend regulieren." (Nanotechnologieverantwortliche, Europäische Kommission, DG Umwelt, 13. November 2008, Übersetzung der Autorin).

Wie solche Maßnahmen inhaltlich ausgestaltet werden und insbesondere ihr Geltungsbereich hängt nicht nur von den von den jeweiligen Mitarbeiterinnen und Mitarbeitern wahrgenommenen Aspekten ab. Vielmehr sind es häufig die Kompetenzbereiche, in welchen die spezifischen Behörden arbeiten, welche die inhaltliche Gestaltung solcher Maßnahmen vorgeben. Dazu erläutert der Vertreter der Generaldirektion Forschung, dass sich der Verhaltenskodex auf die Forschung beschränkt, da diese in der Kompetenz seiner Generaldirektion liegt. Durch die Kompetenzaufteilung unter den Generaldirektionen und der Behandlung der Nanotechnologie in verschiedenen Generaldirektionen, lassen sich übergreifende Regulierungsansätze nur in interdepartementaler Kooperation und Übereinstimmung erzielen:

„Der Verhaltenscodex ist deswegen auf Forschung beschränkt, weil wir über die anderen Aspekte keine Kompetenz haben. Aber es ist nicht so, dass wir glauben, dass es nur für die Forschung wichtig ist. Es ist eine Frage der Kompetenzaufteilung." (Nanotechnologieverantwortlicher, Europäische Kommission, DG Research, 7. Oktober 2008).

Ein Mitarbeiter der Generaldirektion Unternehmen erläutert, dass Forschungsinhalte kaum reguliert sind. Während formale Bereiche wie Arbeitssicherheit, Tierschutz und ethische Aspekte gesetzlich gergelt werden, gibt es für die inhaltlichen Bereiche kaum Gesetze. Deshalb können freiwillige Verhaltenskodizes solche Lücken füllen: 
„Forschung ist oft von der Regulierung ausgeschlossen. Das soll nicht bedeuten, dass es keine Regeln gibt. Da sind immer Regeln anwendbar, Arbeitsschutzgesetze, die viel Betonung auf Dialog und Kommunikation mit den Mitarbeitenden legen und so weiter. Aber man kann auch einem Verhaltenskodex zustimmen und das können Kodizes sein, die von der Industrie entwickelt und umgesetzt werden." (Nanotechnologieverantwortlicher, Europäische Kommission, DG Unternehmen und Industrie, 3. Dezember 2008, Übersetzung der Autorin).

Verschiedene Autoren weisen auf die große Bedeutung hin, die informelle und nicht bindende Regulierungsmaßnahmen auf Unionsebene einnehmen, die mit dem Begriff der ,new Governance' umschrieben werden (siehe Kapitel 1.1). Dies zeigt sich auch in den Interviews, in welchen verschiedene Interviewpartner der Europäischen Kommission ein Verständnis von Regulierung vertreten, das über rein gesetzliche Maßnahmen hinausgeht. Der Vertreter der Generaldirektion Unternehmen erwähnt beispielsweise, dass insbesondere in Bereichen, in welchen sich das Wissen rasch wandelt, neben gesetzlichen Maßnahmen auch informelle Steuerungs- und Lenkungsinstrumente ebenso wie Vorschriften zu Standards, Testmethoden und Richtlinien zur wissenschaftlichen Praxis bedeutsam sind:

„Die derzeitige Regulierung umfasst nichtverbindliche Gesetze. Sie umfasst auch das gesamte Set von Governance-Instrumenten zur Behandlung bestimmter Themen. Was zum einzelnen Gesetz hinzukommt, sind Standards, Testmethoden, übertragene Regulierung, die durch einen Prozess übernommen wird, den wir als Komitologie bezeichnen. Dies umfasst Richtlinien, wissenschaftliche Empfehlungen und die ganze Anlage, die eine reibungslose Implementierung der Gesetze erlaubt. Es gibt Bereiche, die nicht regulierbar sind, weil sich die Dinge bewegen und nicht etabliert sind und damit würde man jeglichen Fortschritt ersticken."(Nanotechnologieverantwortlicher, Europäische Kommission, DG Unternehmen und Industrie, 3. Dezember 2008, Übersetzung der Autorin).

Aus dieser Perspektive stellen für die Interviewpartner der Europäischen Kommission freiwillige Regulierungsmaßnahmen einen wichtigen Aspekt dar. Derselbe Interviewpartner weist darauf hin, dass freiwillige Ansätze einen relevanten Teil der Regulierungsaufgabe betreffen. Auch wenn diese nicht verbindlich sind, entsteht doch ein entscheidender Druck auf deren Anwendung:

„Verhaltenskodizes, freiwillige Systeme sind wichtig. Wir machen unterschiedliche Arten von Standards mit ISO. Das ist auch freiwillig, aber es kann extrem wichtig sein. Es ist Teil des Instrumentariums. Es ist nicht verbindlich aber es wird stark gefördert." (Nanotechnologieverantwortlicher, Europäische 
Kommission, DG Unternehmen und Industrie, 3. Dezember 2008, Übersetzung der Autorin).

Wie der Verantwortliche der Generaldirektion Forschung weiter erläutert, konzentrieren sich private Kodizes häufig auf die spezifischen Bedürfnisse der sie lancierenden Organisationen und funktionieren in Ergänzung zu Regierungsinititativen. Den Vorteil einer Regierungsinitiative sieht er in der Vermeidung einer spezifischen Interessenskonstellation und in einer weiterreichenden Bereichsabdeckung. Deshalb sind in den Verhaltenskodex zur verantwortlichen Nanotechnologieforschung auch Vorschläge eingearbeitet worden, wie die Vorsorge implementiert werden kann:

„Aber die verantwortungsorientierten Verhaltenskodizes, wie auch der prinzipienbasierte Kodex der Royal Society, das sind Initiativen, die sich mehr an die Industrie richten und daher auch in gewissem Sinne auch komplementär zu unserem Kodex sind. Der Vorschlag der Kommission umfasst etwas mehr als die privaten Initiativen. Vor allem im Bereich der Implementierung der Vorsorge." (Nanotechnologieverantwortlicher, Europäische Kommission, DG Research, 7. Oktober 2008).

Beim europäischen Verhaltenskodex handelt es sich um eine der wenigen regulatorischen Maßnahmen, die nicht nur Nanomaterialien, sondern die Nanotechnologie im übergreifenden Sinne betrifft. Die Interviewpartnerin der Generaldirektion Umwelt teilt die Ansicht, dass Kodizes auf Regierungsebene einen umfassenderen Anwendungsbereich abdecken, als es private Initiativen tun können. Dies zeigt sich auch beim Verhaltenskodex der Europäischen Union, der die gesamte Nanotechnologie-Forschung abdeckt; also nicht nur bereits entwickelte Nanomaterialien, sondern auch umfassend die Nanotechnologie als solche und Nanomaterialien zukünftiger Generationen. Ein derart umfassender Rechtsakt kann ihrer Ansicht nach nur auf freiwilliger Basis erfolgen:

„Da wir nicht wissen, was kommen wird und da Forschung nie bestimmen kann, was in der Zukunft kommt, ist es am besten, einen Verhaltenskodex zu entwickeln, der freiwillig sein muss." (Nanotechnologieverantwortliche, Europäische Kommission, DG Umwelt, 13. November 2008, Übersetzung der Autorin).

Der Verhaltenskodex gilt sowohl für private als auch für staatlich finanzierte Forschung. Er bezieht sich auf die Mitgliedsstaaten und auf vielfältige Anspruchsgruppen. Dieser breite Gültigkeitsbereich ist jedoch auch Gegenstand verschiedener Kritiken. Insbesondere Forscherinnen und Forscher fühlen sich als Zielgruppe dieser Maßnahme im Vergleich zur Industrie benachteiligt und erachten die Maßnahme deshalb als ungerecht (vgl. 
Lacour 2010). Zudem bleibt der Verhaltenskodex inhaltlich relativ unbestimmt. In diesem Zusammenhang übt ein Parlamentsvertreter grundsätzliche Kritik an freiwilligen Maßnahmen. Er vertritt die Überzeugung, dass freiwillige Maßnahmen kaum über die bestehende gesetzliche Regulierung hinausgehen und dass die zentrale Motivation ihrer Etablierung weniger darin liegt, potenzielle Risiken auszuschließen, als vielmehr eine gesetzliche Regulierung zu verhindern:

„Meine Erfahrung hier in Brüssel mit freiwilligen Vereinbarungen oder Initiativen ist negativ. Diese werden fast ausschließlich vorgelegt, um zu verhindern, dass der Gesetzgeber aktiv wird. Das heißt, es sind weniger Maßnahmen zur Gefahrenabwehr als vielmehr solche zur Regulierungsabwehr. Mir ist keine freiwillige Vereinbarung bekannt, die sinnvoll über das bestehende Recht hinausarbeitet. Meistens werden Dinge, die im Interesse der Firmen sind, zugestanden und die wesentlichen Probleme ausgeblendet." (Nanotechnologiespezialist und Abgeordneter, Europäisches Parlament, 30.10.2008).

Das Parlament fordert auch politische und institutionelle Reaktionen, beispielsweise die Etablierung eines Koordinators für Nanotechnologie, der die Politiken der verschiedenen Generaldirektionen vernetzen soll (P6_TA 2009). Die ebenfalls vom Parlament kritisierte fehlende Einigung über die Definition von Nanomaterialien wird von der Kommission im Jahr 2011 durch die Publikation einer Mitteilung zur Empfehlung einer Definition für Nanomaterialien entsprochen (COM 2011).

Die Empfehlung der Kommission für die Definition von Nanomaterialien basiert inhaltlich auf dem Ansatz, der sich bereits früher als der einzige gemeinsame Nenner im Definitionsdiskurs herausgestellt hat; nämlich auf der Partikelgröße und nicht auf den von den jeweiligen Materialien ausgehenden Reaktionsweisen, Implikationen oder deren Verhalten (vgl. Lösch 2009). Nach dem Vorschlag der Europäischen Kommission sollen unter dem Begriff der ,Nanomaterialien' Materialien verstanden werden, deren Hauptbestandteile eine Größe zwischen 1 und 100 Milliardstel Metern haben:

„Ein Nanomaterial ist ein natürliches, bei Prozessen anfallendes oder hergestelltes Material, das Partikel in ungebundenem Zustand, als Aggregat oder als Agglomerat enthält, und bei dem mindestens die Hälfte der Partikel in der Anzahlgrößenverteilung ein oder mehrere Außenmaße im Bereich von $1 \mathrm{~nm}$ bis $100 \mathrm{~nm}$ haben." (COM 2011).

Ziel dieser Empfehlung ist es, einer dem Europäischen Parlament im Jahr 2009 gemachten Zusage nachzukommen, eine Definition vorzulegen, die umfassend auf alle nanomaterialienspezifische europäische Rechtsvor- 
schriften angewendet werden kann (COM 2011). Die Definition stützt sich auch auf Gutachten des wissenschaftlichen Ausschusses SCENIHR und der Forschungsstelle (Joint Research Center, JRC). Die Mitteilung der Kommission zur Definition von Nanomaterialien ist vor ihrer Publikation einer öffentlichen Konsultation unterzogen worden. ${ }^{113}$ Wie die Empfehlung zum Forschungskodex ist auch diejenige für die Definition von Nanomaterialien rechtlich nicht verbindlich.

\subsubsection{Die gesetzliche Regulierung: Das sektorale Produktrecht}

Seit dem Jahr 2009 kommt es auf Unionsebene vermehrt in verbindlichen Rechtsakten zu nanospezifischen Regelungen, die insbesondere das sektorale Produktrecht betreffen, wie das Kosmetik-, das Lebensmittel- sowie das Chemikalien- und Stoffrecht. Im Bereich des Chemikalien- und Stoffrechts werden nanospezifische Anpassungen an die REACH-Verordnung und an die Verordnung zur Einstufung, Kennzeichnung und Verpackung von Stoffen und Gemischen (Classification, Labelling and Packaging of substances and mixtures, CLP) diskutiert, verhandelt und auf der Implikationsebene auch umgesetzt (vgl. Eisenberger, et al. 2010). Auch im Rahmen der Revision der Richtlinie zur Beschränkung der Verwendung gefährlicher Stoffe (Restriction of Hazardous Substances, RoHS-Richtlinie) fordert das Europäische Parlament im Jahr 2011 die Aufnahme nanospezifischer Regulierungen. ${ }^{114}$ Wie ein Interviewpartner der Generaldirektion Forschung erläutert, werden Anpassungen von Richtlinien in der Europäischen Kommission themenspezifisch in Zusammenarbeit mit den Abgeordneten der involvierten Departemente erarbeitet:

„Die Kommission ist so organisiert, dass sich bei den Anpassungen von Richtlinien, die Mitarbeitenden der für die jeweiligen Richtlinien verantwortlichen Departemente also z.B. bei REACH das DG Umwelt mit Vertretern von Mitgliedsstaaten treffen." (Nanotechnologieverantwortlicher, Europäische Kommission, DG Research, 7. Oktober 2008).

113 Siehe http://europa.eu/rapid/press-release_IP-11-1202_de.htm (15.05.2014).

114 Die Ergänzung der Lebensmittelzusatzstoffverordnung sieht insbesondere die auf Ende 2014 in Kraft tretende Kennzeichnung nanospezifischer Lebensmittel vor. Die Anpassung der RoHS-Richtlinie erfolgt im Rahmen von zwei eher als marginal bezeichneten Provisionen (vgl. Eisenberger, et al. 2010). 
Im Jahr 2009 ist die Lebensmittelzusatzstoffverordnung und im Jahr 2010 sind Teile der neuen Kosmetikverordnung in Kraft getreten. Die Kosmetikrichtlinie ist nicht aufgrund des Regulierungsdiskurses über Nanomaterialien überarbeitet worden. Vielmehr hat eine ohnehin anstehende Revision dazu geführt, dass Nanomaterialien mitberücksichtigt worden sind (vgl. Eisenberger, et al. 2010). Im gleichen Jahr ist die geplante Verordnung über neuartige Lebensmittel gescheitert, die ebenfalls nanospezifische Regelungen vorgesehen hat (ibid.). Zum Zeitpunkt des Interviews mit dem Abgeordneten des Europäischen Parlaments hat dieses gerade die Kosmetikrichtlinie verhandelt. Dazu erläutert der Interviewpartner die Position des Parlaments, das analog zur Regulierungspraxis bei Arzneimitteln eine Sicherheitsprüfung für alle Nanomaterialien enthaltenden Kosmetika als Bedingung für die Marktzulassung verlangt: 115

„Im Wesentlichen fordern wir eine Sicherheitsprüfung vor der Vermarktung. Bei der Kosmetik gibt es je nach Inhaltsstoff unterschiedliche Herangehensweisen. Es ist nicht so wie bei den Pharmaka, wo eine Zulassung da sein muss, bevor sie auf den Markt kommen. Das ist bei Kosmetika mit Ausnahme der Positivlisten für UV-Filter und Konservierungsmittel anders. Für Nano in Kosmetika möchten wir eine Sicherheitsbewertung vor der Vermarktung sicherstellen.“ (Nanotechnologiespezialist und Abgeordneter, Europäisches Parlament, 30.10.2008).

Zudem erwähnt er, dass aus seiner Sicht verbrauchernahe und umweltoffene Nanomaterialien enthaltende Produkte nicht vermarktet werden dürfen, bis robuste Testinstrumente vorhanden sind. Produkte sollen erst dann auf den Markt gelangen, wenn es möglich ist, ihre Sicherheit ausreichend nachzuweisen:

„Solange die adäquaten nanospezifischen Testmethoden zur Risikoabschätzung fehlen, schätze ich das Risiko dieser Anwendungen im Vergleich zu ihrem Nutzen höher ein. Deshalb sollte diese Technologie in allen verbrauchernahen und umweltoffenen Bereichen nicht zum Einsatz kommen. Man sollte die nötigen Bewertungsinstrumente haben, bevor man etwas auf den Markt bringt. Letzten Endes ist das die Anwendung des allgemein akzeptierten Prinzips: 'No data, no market'.“ (Nanotechnologiespezialist und Abgeordneter, Europäisches Parlament, 30.10.2008).

Im Jahr 2012 hat die Kommission eine Biozidprodukteverordnung mit nanospezifischen Regelungen erlassen (COM 2011). Trotz der nach dem

115 Die Sicherheitsprüfung gilt für sämtliche Arzneimittel, nicht nur für solche, die Nanomaterialien enthalten (EC 726 2004). 
Jahr 2009 beobachtbaren Tendenz einer verstärkten Produktorientierung und gesetzlichen Regulierung, ist das für die Regulierung von Nanomaterialien bedeutsamste Gesetz, die REACH-Verordnung, bislang keiner die Nanomaterialien explizit berücksichtigenden Revision unterzogen worden (vgl. Eisenberger, et al. 2010).

\subsubsection{Zusammenfassung Europäische Union: Regulieren oder implementieren?}

Wie im Abschätzungsdiskurs sind auch im Regulierungsdiskurs auf Unionsebene als Akteure hauptsächlich die beiden europäischen Institutionen Kommission und Parlament beteiligt. In der Kommission sind insbesondere die Generaldirektionen Umwelt, Forschung \& Innovation, Unternehmen \& Industrie und Gesundheit \& Verbraucher involviert. Wichtig sind auch die Europäische Chemikalienagentur ECHA und das für die Regulierung von Nanomaterialien eigens eingesetzte Gremium aus den zuständigen Länderbehörden, die REACH-Competent Authorities (CA).

Ein wesentlicher thematischer Schwerpunkt des Regulierungsdiskurses auf Unionsebene betrifft die Chemikalienregulierung REACH. Hierzu ist zwischen dem Parlament und der Kommission eine Kontroverse bezüglich der Frage entstanden, ob die potenziellen Umwelt-, Gesundheits- und Sicherheitsimplikationen von Nanomaterialien unter der bestehenden Umwelt- und Chemikalienregulierung REACH ausreichend abgedeckt sind. Während die für die Implementierung der Nanotechnologiepolitik zuständige Kommission die Auffassung vertritt, dass die REACH-Verordnung für die regulatorische Behandlung von Nanomaterialien ausreicht und der Handlungsbedarf eher bei der Implementierung verortet, verneint dies das Parlament. Dieses äußert sich besorgt über die Diskrepanz zwischen der mangelnden Verfügbarkeit von Informationen über potenzielle nachteilige Umwelt-, Gesundheits- und Sicherheitsimplikationen angesichts der beträchtlichen Anzahl der bereits am Markt verfügbaren Nanomaterialien und fordert deren spezifische Regulierung.

Aus Sicht des Parlamentes generiert die fehlende gesetzliche Grundlage substanzielle Fragen über die Kompetenz der bestehenden Regulierung, mit komplexen Spitzentechnologien wie mit Nanotechnologien umzugehen. Während das Parlament das zentrale Defizit beim fehlenden Rechtsrahmen verortet, vertritt die Kommission die Haltung, dass dem Problem auf Implementierungsebene zu begegnen sei. Die Kommission stützt ihre 
Argumentation auf ihre Auffassung der Substanzäquivalenz von Nanomaterialien und Chemikalien. Demgegenüber stellen Nanomaterialien aus Sicht des Parlaments neuartige Substanzen dar, deren spezifische Eigenschaften vorsorgeorientiert auf der Gesetzesebene reguliert werden müssen. Das Vorsorgeprinzip würde es ermöglichen, unter der REACH-Regulierung neben toxikologisch nachweisbaren Parametern auch nicht wissenschaftlich erhärtete Hinweise auf ein mögliches Gefährdungspotenzial von Nanomaterialien zu regulieren. Daraus wird jedoch kein Dispositiv abgeleitet. Das heißt, dass sich eine solche Strategie im Rahmen von REACH nicht feststellen lässt.

Das im europäischen Regulierungsdiskurs thematisierte Nichtwissen bezieht sich also insbesondere auf Fragen, wie die gesetzliche Behandlung von Nanomaterialien unter REACH, die Definition von Nanomaterialien, die Entwicklung von adäquaten Testmethoden, die Verfügbarkeit von Nachweisen für konkrete Risiken und ethische Aspekte. Diese breite Verhandlung von Nichtwissen im Diskurs auf der Unionsebene betrifft also nicht nur das mit den potenziellen Umwelt-, Sicherheits- und Gesundheitsauswirkungen (EHS) verbundene Nichtwissen bei Nanomaterialien. Vielmehr besteht auch eine umfangreiche Unschlüssigkeit bezüglich der zu ergreifenden Maßnahmen.

Praktiken, die sich im Regulierungsdiskurs auf Unionsebene gezeigt haben, umfassen die von der Kommission verfasste Publikation einer Regulierungsanalyse und ebenfalls von der Kommission eingesetzte freiwillige Maßnahmen wie einen Verhaltenskodex zur verantwortungsvollen Forschung und eine Empfehlung zur Definition von Nanomaterialien. Damit etabliert die Kommission als einzige der untersuchten Behörden einen übergreifenden Rechtsakt zur Forschung, der sich nicht alleine auf die Nanomaterialien beschränkt, sondern auch die Nanowissenschaften und die Nanotechnologie miteinschließt und der ethische Aspekte behandelt. Mit der Publikation des Verhaltenskodexes will die Kommission in der Forschung auf Unionsebene und in den Mitgliedsstaaten ein erhöhtes Verantwortungsbewusstsein generieren. Auch lässt sich hier erneut das bereits im Abschätzungsdiskurs beobachtete Dispositiv der Integration der Nanomaterialien in die bestehenden Strukturen nachweisen. Dazu hat die Kommission Bereiche des sektoralen Produktrechts wie Lebensmittel und Kosmetika mit nanospezifischen Regelungen angepasst und eine Empfehlung zur einheitlichen Definition von Nanomaterialien publiziert.

Dennoch lassen sich im Regulierungsdiskurs auch neue, spezifisch auf die Nanotechnologie fokussierende Maßnahmen beobachten. Die Europäi- 
sche Kommission hat ihren Schwerpunkt in der regulatorischen Behandlung der Nanotechnologie zwischen den Jahren 2004 und 2009 - in Übereinstimmung mit der von verschiedenen Autoren beschriebenen zunehmenden regulatorischen Bedeutung von sogenannt, weichen' oder ,new Governance' Maßnahmen auf Unionsebene (Kapitel 3.3.2) - hauptsächlich auf nicht bindende Ansätze wie z.B. den Verhaltenskodex gelegt. Möglicherweise auch im Zusammenhang mit dem zunehmenden Druck des Parlaments bezüglich des Chemikalienrechts hat die Union diese Strategie durch einige auf spezifische Anwendungen des sektoralen Produktrechts beschränkte gesetzliche Regulierungsinitiativen (z.B. Kosmetikund Lebensmittelrecht) ergänzt (siehe oben).

Die im Rahmen der vorliegenden Studie erhobenen Daten deuten darauf hin, dass das im europäischen Umweltrecht und darin insbesondere in REACH zentral verankerte Vorsorgeprinzip auf Nanomaterialien kaum angewendet wird. Vielmehr lässt sich auf der Unionsebene die mit dem USamerikanischen Ansatz vergleichbare Praxis der inkrementellen Regulierung feststellen: Statt die Nanomaterialien in einem eigenen Gesetzesakt oder im Rahmen der Chemikaliengesetzgebung REACH umfassend gesetzlich zu regulieren, werden Nanomaterialien über ihre Anwendungen und Produkte, wie Kosmetika, Lebensmittel und Biozide medienspezifisch reguliert und die Anhänge des Chemikalienstatuts REACH angepasst. Für die Behandlung darüber hinausgehender Nichtwissensfragen wählt die Europäische Union ebenso, wie es sich in den Vereinigten Staaten beobachten ließ, die Form des nichtverbindlichen Rechtsakts. Während die Vereinigten Staaten dabei ihren Schwerpunkt auf ein freiwilliges Datenmeldeverfahren unter der Chemikaliengesetzgebung TSCA legen, fokussiert die Europäische Union die Forschungsethik und die begriffliche Definition von Nanomaterialien.

\subsection{Großbritannien: Ein freiwilliges Datenmeldeverfahren und eine unübliche Kooperation}

Im Zentrum des britischen Regulierungsdiskurses steht wie auf Unionsebene die Frage nach der Regulierung von Nanomaterialien im Stoffrecht. Hier ist insbesondere das von der britischen Umwelt- und Gesundheitsbehörde DEFRA im Jahr 2007 eingeführte freiwillige Datenmeldeverfahren bedeutsam. Dieses ist demjenigen der US-amerikanischen EPA Pate gestanden und kämpft mit denselben Schwierigkeiten. Diese umfassen eine 
— im Vergleich zur Anzahl der sich bereits auf dem Markt befindendenden Nanomaterialien - bescheidene Rücklaufquote und die Unklarheit über seine Weiterführung. Ebenfalls vertieft diskutiert wird in diesem Kapitel eine regierungsexterne freiwillige Maßnahme: der ,responsible Nano Code' - hier kurz als Nano Code bezeichnet, der vergleichbar mit dem EDF - DuPont Schema ebenfalls in einer eher unüblichen Kooperation zwischen einer Wissenschaftsakademie und einer Investitionsberatungsfirma etabliert worden ist. Wie die meisten prinzipienbasierten Verhaltensanweisungen kämpft auch der Nano Code mit verschiedenen Schwierigkeiten. Durch seine Entwicklung im Rahmen eines Kompromisses aus unterschiedlichen Interessen zeichnet er sich durch vage Formulierungen aus und durch seine inhärent freiwillige Orientierung fehlt ihm die rechtliche Verbindlichkeit. Wie im Abschätzungsdiskurs stehen auch im Regulierungsdiskurs Großbritanniens hauptsächlich Implikationen im Zentrum. Trotz dieses starken Fokus auf die Technikfolgen, wird die britische Regierung ebenso wie die Regierungen der anderen untersuchten Länder darin kritisiert, zuwenig zur Vermeidung potenziell nachteiliger Auswirkungen von Nanomaterialien zu tun.

\subsubsection{Nanomaterialien im Stoffrecht}

Wie auf der Unionsebene und in den anderen untersuchten Nationen wird auch im britischen Regulierungsdiskurs die zentrale Herausforderung in der Frage nach der Berücksichtigung der Nanotechnologie im Rahmen der bestehenden Regulierung gesehen. Ebenfalls problematisiert wird die mehrdeutige Definition von Nanotechnologie und Nanomaterialien (vgl. Marchant, et al. 2007,190). Ferner stehen Unsicherheiten über potenzielle Risiken, die Frage nach der Gleichbehandlung mit anderen Technologien sowie die Berücksichtigung von sozialen und ethischen Risiken in der Sicherheits-, Umwelt- und Gesundheitsregulierung im Zentrum des Diskurses (vgl. Kearnes \& Doubleday 2010). Zudem sieht sich auch die britische Regierung vor die Schwierigkeit gestellt, den Regulierungsgegenstand einer schwer eingrenzbaren und sich laufend weiterentwickelnden Technologie festzulegen. Insbesondere sollte sowohl eine Unter- als auch eine Überregulierung vermieden werden und eine potenzielle Regulierung die Balance zwischen der Verhinderung möglicher nachteiliger Implikationen und der Förderung einer potenziell lukrativen Technologie gewährleisten (ibid.). 
Ein wichtiger Akteur im britischen Regulierungsdiskurs ist die- Umwelt, Ernährungs- und Landwirtschaftsbehörde DEFRA. Neben der Einberufung von Expertengremien hat sie verschiedene Studien zuhanden der in ihrer Verantwortung stehenden Nanotechnologieforschungskoordinationsgruppe (NRCG) veröffentlicht (siehe Kapitel 2.4). In diesen beschreibt sie die Forschungs- und Regulierungslücken in den Bereichen Umwelt, Gesundheit und Sicherheit (EHS). ${ }^{116}$ Zudem empfiehlt die DEFRA die Schaffung eines Nanomaterialienregisters. Hauptsächliche Regulierungslücken identifiziert sie bei der Europäischen Chemikalienregulierung REACH und ihrem Geltungsbereich für jährliche Produktionsmengen von über einer Tonne. Da sich die Produktionsvolumina von Nanomaterialien in der Regel im Gramm- oder maximal im Kilogrammbereich befinden, sind diese durch REACH größtenteils nicht abgedeckt, wie ein Interviewpartner der Umweltbehörde erläutert. Im Unterschied zu seinen Kollegen der Europäischen Kommission, vertritt er die Meinung, dass eine regulatorische Anpassung von REACH unumgänglich ist. Für ihn besteht die Frage lediglich darin, wie diese zu erfolgen hat:

„Da sind offene Fragen bezüglich der Grenzwerte. Dies ist eine weite und komplexe Angelegenheit und klar, wenn die Grenzwertregel bei allen Stoffen aufgehoben würde, dann hätten wir ein Problem, weil es 100'000 Chemikalien gibt, die begutachtet werden müssten und schon so ist die Arbeitsbelastung der Europäischen Chemikalienagentur beträchtlich. Es wird in Europa einige wohlüberlegte Diskussionen darüber geben müssen, wie REACH genau korrigiert werden muss. Und in drei oder vier Jahren werden wir hoffentlich eine europäische Chemikalienregulierung haben, welche die Probleme um die Nanomaterialien anspricht." (Nanotechnologiespezialist, Department of Environment, Food and Rural Affairs/DEFRA, 7.11.2008, Übersetzung der Autorin).

Die im Interview geäußerte Hoffnung, im Jahr 2011 oder 2012 eine auf Nanomaterialien angepasste Europäische Chemikalienregulierung vorliegen zu haben, hat sich bis zum Abschluss der vorliegenden Erhebung im Dezember 2014 nicht erfüllt.

Unter Berücksichtigung der im Royal Society Bericht und in verschiedenen Nachfolgestudien festgestellten Regierungslücken hat die britische Umweltbehörde im Jahr 2007 ein freiwilliges Datenmeldeverfahren für synthetische Nanomaterialien (VRS) publiziert. Das Verfahren sieht die

$116 \mathrm{Zu}$ den verschiedenen Studien siehe beispielsweise (Aitken, et al. 2009; Chaudhry, et al. 2005a; Chaudhry, et al. 2005b; DEFRA 2006; HM Government 2006; HM Government 2007a). 
freiwillige Meldung von Daten durch Firmen und Organisationen vor, welche in die Herstellung, die Verwendung, den Import, die Forschung und die Entsorgung von nanoskaligen Materialien involviert sind. Ziel der DEFRA ist es, mittels der im Rahmen des Systems gemeldeten Daten ein besseres Verständnis der Eigenschaften und der Charakteristika der in Großbritannien hergestellten und verwendeten synthetischen Nanomaterialien zu erhalten. Zudem sollen aufgrund der gewonnenen Daten potenzielle Umwelt- und Gesundheitsrisiken, Sicherheitsfragen und die Exposition von Arbeitnehmern und Konsumenten besser berücksichtigt werden (HM Government 2007b). ${ }^{117}$ Der in die Entwicklung des Datenmeldeverfahrens involvierte Interviewpartner der Umweltbehörde erläutert die Hintergrundüberlegungen zum VRS. Diese basieren insbesondere auf dem Wunsch der DEFRA nach einem Marktüberblick:

„Das VRS entstand hauptsätzlich aus der Idee, dass die britische Industrie viel nanowissenschaftliche Forschung durchführt. Wenn sie nun Daten über die Toxizität oder die Form und die Zusammensetzung von Nanomaterialien generiert, wäre es gut, wenn sie diese mit uns teilen würde. Das würde uns helfen, unsere eigene Beweisgrundlage und unser eigenes Forschungsprogramm zu ergänzen. Wenn man wissen will, welche Nanoprodukte auf dem Markt sind, dann muss man wirklich mit der Industrie sprechen.“ (Nanotechnologiespezialist, DEFRA, 7.11.2008, Übersetzung der Autorin).

Wie in den anderen untersuchten Ländern werden auch in Großbritannien Regulierungslücken festgestellt. Solche umfassen die in der Royal Society Studie beschriebenen Bedenken bezüglich der regulatorischen Abdeckung der sich rasch auf dem Markt ausbreitenden Nanomaterialien. Verschiedene Interviewpartner bringen die Lancierung des Datenmeldeverfahrens mit den wahrgenommenen Regulierungsdefiziten zusammen. Dazu stellt ein Interviewpartner des nationalen Forschungsrats für Ingenieurs- und Physikwissenschaften (EPSRC) fest, dass das freiwillige Schema einen Ansatz im Umgang mit einer Regulierungslücke darstellt:

„Gewissermaßen war das freiwillige Meldesystem ein Symptom, das aus einem Regulierungsvacuum entstand." (Nanotechnologiespezialist, EPSRC, 2.12.2008, Übersetzung der Autorin).

Im Jahr 2009 veröffentlicht die DEFRA eine Evaluation ihres seit zwei Jahren laufenden freiwilligen Meldeverfahrens (VRS). Die Anzahl der ge-

117 Siehe auch http://archive.DEFRA.gov.uk/environment/quality/nanotech/policy.ht $\mathrm{m}(29.6 .2011)$. 
meldeten Nanomaterialien beläuft sich auf dreizehn. Diese Rückmeldequote wird von verschiedener Seite als enttäuschend bezeichnet. Diese Enttäuschung wird vom Nanotechnologiekonsumprodukteinventar des Wilson Zentrums unterstützt, das in diesem Zeitraum von über 50 sich auf dem Markt befindenden Nanotechnologieprodukten alleine in Großbritannien ausgeht. ${ }^{118}$ Dies führt zu verschiedenen Kritiken am VRS. Der Vertreter des EPSRC zieht den Schluss, dass das freiwillige Datenmeldeverfahren ein Misserfolg ist. Er bewertet das Schema als unklar konzipiert, so dass die Firmen keinen Anreiz darin sehen, ihre Daten zu melden:

„Das freiwillige Meldeschema der DEFRA wird weitherum als Fehlschlag betrachtet. Ein entmutigender Misserfolg, wirklich; da sich niemand darum bemüht hat, irgendetwas zu melden. Es entstand der Eindruck, dass es falsch aufgefasst wurde. Die Leute waren sich nicht im Klaren darüber, weshalb sie irgendetwas melden sollten.“ (Nanotechnologiespezialist, EPSRC, 2.12.2008, Übersetzung der Autorin).

Der verantwortliche Leiter der DEFRA gibt sich ebenfalls enttäuscht über die geringe Rückmeldequote:

„Ja, da stehen wir jetzt zwei Jahre später mit 13 Meldungen da; es ist ein bisschen enttäuschend." (Nanotechnologiespezialist, DEFRA, 7.11.2008, Übersetzung der Autorin).

Gleichzeitig versucht er, diese zu rationalisieren. Eine Schwierigkeit sieht er bei der Pionierhaftigkeit des britischen Ansatzes. Seine Behörde hat das System von Grund auf neu entwickelt und fortlaufend Anpassungen und Überarbeitungen vorgenommen. Daraus ist ein umfangreiches Raster entstanden, das die Firmen möglicherweise alleine schon durch seinen Umfang davor abgeschreckt hat, ihre Daten zu melden:

„Es war das erste Schema weltweit und daher, als wir es lancierten, erstellten wir eine ziemlich umfangreiche Datenliste. Sie umfasste insgesamt um die 10 Seiten. [...] Die Industrie, die ein spezifisches Nanomaterial für eine spezifische Eigenschaft auswertet, versteht nicht notwendigerweise alles über sein toxikologisches Profil und ich denke, dass viele Firmen durch den Umfang der benötigten Daten eingeschüchtert waren." (Nanotechnologiespezialist, DEFRA, 7.11.2008, Übersetzung der Autorin).

Zudem bringt er die geringe Rückmeldequote mit dem fehlenden Bewusstsein in bestimmten Branchen in Verbindung. Branchen, die traditionellerweise mit nanoskaligen Materialien arbeiten, wie die Farbindustrie, reali-

118 Siehe http://www.nanotechproject.org/inventories/consumer/ (29.6.2011). 
sieren möglicherweise gar nicht, dass sie zur Nanotechnologieindustrie gehören und sind sich nicht darüber im Klaren darüber gewesen, dass das VRS auch sie betrifft:

„Viele Firmen sind sich dessen gar nicht bewusst, dass sie Nanotechnologie machen, oder sie sind sich nicht bewusst, dass sie Nanomaterialien verarbeiten. Klar, die Farbindustrie benutzt Nanomaterialien in Farben, aber das machen sie schon seit Jahren. Es ist schwierig, so jemanden dazuzubringen, von sich selbst zu denken, dass er ein Nano-Hersteller ist und an Regierungsprogrammen für Nanomaterialien teilnehmen sollte." (Nanotechnologiespezialist, DEFRA, 7.11.2008, Übersetzung der Autorin).

Insgesamt bleiben jedoch die Gründe, die zu der geringen Rücklaufquote geführt haben, unklar. Der DEFRA-Vertreter interpretiert die Quote als Abbild der kommerziellen Realität einer sich in Entwicklung befindenden Industrie. Er denkt aber gleichwohl über den Nutzen eines obligatorischen Schemas nach:

„Ein weiterer Zwek des Schemas war es, das Ausmaß der Kommerzialisierung herauszufinden. So hat das Schema sicher gezeigt, dass gar nicht so viel passiert. In einem frühen Stadium der Nanotechnologie ist vielleicht eine Rückmeldung von 13 gar nicht so weit davon entfernt, was man erwarten dürfte. Deshalb ist eine der Optionen weiterzufahren wie bisher. Aber klar, wir überlegen uns auch obligatorische Ansätze." (Nanotechnologiespezialist, DEFRA, 7.11.2008, Übersetzung der Autorin).

Die Vermutung, dass die 13 Meldungen durchaus die kommerzielle Realität abbilden, wird von einer Branchenvertreterin geteilt. Sie zieht den Vergleich zum US-amerikanischen Datenmeldeverfahren, welches ebenfalls eine geringe Meldequote verzeichnet:

„Und das andere ist, dass die 13 Meldungen durchaus kommerzielle Realität sind. Wir haben dasselbe in den Vereinigten Staaten beobachtet, die Rückmeldungen auf das Voluntary Reporting Scheme der EPA liegen auch weit unter dem, was sie erwartet haben." (Nanotechnologieverantwortliche, NIA, 5.11.2008).

Die Defizite von nichtverbindlichen Rechtsakten werden wie in den anderen untersuchten Ländern auch in Großbritannien hauptsächlich in zwei Bereichen verortet: In ihrer Freiwilligkeit und in der Sicherstellung der kommerziell notwendigen Vertraulichkeit. In einem hoch kompetitiven Feld führt die Freiwilligkeit dazu, dass die Firmen in der Publikation ihrer Daten zurückhaltend sind und dass sie sich kaum die Zeit nehmen, die Dokumente auszufüllen. Die Vertreterin einer Konsumentenschutzorganisati- 
on wünscht sich aus diesem Grund, dass das freiwillige Datenmeldeverfahren in ein obligatorisches System umgewandelt wird:

„Bei dieser Art freiwilliger Datenmeldeschemen werden die Firmen keine Daten melden. Es handelt sich hier um ein höchst kompetitives Feld, in welchem wir nicht sehen, weshalb sich die Firmen verpflichtet fühlen sollten, freiwillig Informationen bereitzustellen. Deshalb wünschen wir uns ein obligatorisches Datenmeldeverfahren." (Nanotechnologieverantwortliche, WHICH, 12.1.2009, Übersetzung der Autorin).

Der Vertreter einer Umweltorganisation teilt diese Ansicht, dass die Industrie die vorhandenen Daten nur kommuniziert, wenn sie mittels eines obligatorischen Meldeverfahrens dazu gezwungen wird und wenn ein solches die Vertraulichkeit gewährleistet:

„Ich bin sicher, dass in den Akten der Industrie eine Menge zusätzlicher Informationen vorhanden ist. Die Firmen produzieren diese Informationen, das ist öffentlich zugänglich. Und ich denke, dass es eine obligatorische Meldung von Sicherheitsdaten und von Resultaten von Sicherheitstests geben muss einige Hinweise, natürlich in einem gewissen Maß innerhalb der Grenzen der kommerziellen Geheimhaltung." (Nanotechnologiespezialist, Greenpeace, 27.11.2008, Übersetzung der Autorin).

Neben seiner Freiwilligkeit wird das Versagen des VRS ähnlich wie beim US-amerikanischen Datenmeldeprogramm auch auf die Komplexität der verlangten Daten zurückgeführt. Eine Branchenverbandsvertreterin erwähnt zudem Fragen des geistigen Eigentums, die eine Rolle gespielt haben mögen:

„Es ist nicht so, dass diese Firmen die Daten nicht haben, wenn sie mit Nanopartikeln arbeiten. Aber man musste zwölf Seiten detaillierte Fragen ausfüllen, wofür Firmen einfach nicht die Ressourcen haben. Zudem ist verlangt, echte Daten und echte Herstellungsverfahren etc. ganz genau transparent zu machen, das ist Information, die Firmen nicht geben können, weil sie dann ihr geistiges Eigentum verlieren würden." (Nanotechnologieverantwortliche, NIA, 5.11.2008).

Wie in den Vereinigten Staaten wird auch in Großbritannien die Einschätzung vertreten, dass ein großer Teil der Nanotechnologiehersteller kleine und oftmals neugegründete Firmen sind. Wie der Vertreter der Umweltbehörde erläutert, können sich diese kaum die Zeit nehmen, Daten für die Behörden zusammenzustellen, wenn sie nicht dazu verpflichtet werden:

„Es war ein freiwilliges Schema und da gibt es Schwierigkeiten, die Industrie dazu zu bringen, an etwas teilzunehmen, an dem sie nicht müssen. Viele dieser Nanomaterialienhersteller sind kleine Firmen in einem frühen Stadium. Sie haben nicht viel Zeit, Daten für die Regierung zusammenzutragen, insbe- 
sondere, wenn es sich nicht um eine obligatorische Auflage handelt." (Nanotechnologiespezialist, DEFRA, 7.11.2008, Übersetzung der Autorin).

In ihrer im Jahr 2010 veröffentlichten Nanotechnologiestrategie stellt die britische Regierung in Aussicht, ein Nachfolgeprogramm zum Datenmeldeverfahren VRS einzuführen, in welches nicht nur Materialien, sondern auch Produkte miteinbezogen werden (HM Government 2010). Dazu sollte eine Arbeitsgruppe aus Vertreterinnen und Vertreter der Wirtschaftsbehörde (IS), der Umweltbehörde (DEFRA), der Gesundheitsbehörde (DH), der Lebensmittelbehörde (FSA) und der Gesundheitsbehörde (HSE) eingesetzt werden. Zudem kündigt die Regierung an, die auf der Unionsebene vollzogenen Gesetzesänderungen $\mathrm{zu}$ neuen Lebensmitteln (novel food), Kosmetik und potenzielle Anpassungen von REACH zu evaluieren und zu begleiten. Zudem sieht die Regierung die Notwendigkeit darin, die Gesetze in den Bereichen ,Medizin und Gesundheitsprodukte' anzupassen (vgl. auch Kapitel 2.4). ${ }^{119}$

\subsubsection{Der Royal Society - Insight Investment Responsible Nano Code}

Wie in den Vereinigten Staaten hat das wahrgenommene Regulierungsdefizit auch in Großbritannien zu regulatorischen Aktivitäten von Nichtregierungsakteuren geführt. Auch in Großbritannien finden eher unübliche Akteure zu einer Kooperation zusammen: Nämlich eine der renommiertesten nationalen naturwissenschaftlichen Akademien; die Royal Society mit der relativ unbekannten britischen Investitionsberatungsfirma Insight Investment, dem neu gegründeten Branchenverband der Nanotechnologieindustrie (Nanotechnology Industries Association, NIA) ${ }^{120}$ und der vom Handelsministerium (DTI) finanzierten Initiative Nanotechnology KTN . Diese vier Akteure entwickeln in der Folge einen ethisch geprägten prinzipienbasierten Verhaltenskodex, den Verantwortungsvollen Nanokodex (Responsible Nano Code, RNC).

119 Ein solches Nachfolgeprogramm zum VRS ist bis zum Abschluss dieser Erhebung im Jahr 2014 nicht eingesetzt worden.

120 Die NIA wird im Jahr 2005 mit Geldern der britischen Regierung als angeblich erster Branchenverband im NT Bereich gegründet. Sie vertritt Hersteller-, Forschungs- und Entwicklungsfirmen, sowie den Handel (Quelle Interview mit Steffi Friedrichs, NIA, 31.10.2008). 
Die Kooperation beginnt mit einem Arbeitstreffen, das die Royal Society mit der Investitionsberatungsfirma Insight Investment im Jahr $2007 \mathrm{zu}$ wirtschaftlichen Aspekten und der verantwortlichen Entwicklung der Nanotechnologie organisiert hat. Insight Investment hat zuvor in Nanotechnologiefirmen eine Umfrage zur Unternehmensverantwortung durchgeführt. Zu diesem Arbeitstreffen haben die beiden Organisationen eine Reihe privatwirtschaftlicher Schlüsselakteure eingeladen. Wie der Vertreter des nationalen Forschungsrats ausführt, ist die Idee zu diesem Treffen aus dem Bewusstsein heraus entstanden, dass insbesondere die von der Royal Society und der Royal Academy of Engineering (2004) verfassten Empfehlungen nach Dialogen von forschungspolitischen Gremien und der Regierung gut aufgenommen worden sind, die Industrie jedoch relativ zurückhaltend geblieben ist:

„Da entstand der Eindruck, dass der private Sektor sich sehr bedeckt hält und versucht, überhaupt nicht in diese Diskussionen einbezogen zu werden. Deshalb hat die Royal Society ein Arbeitstreffen organisiert, worin sie verschiedene Akteure des privaten Sektors zusammengebracht hat." (Nanotechnologiespezialist, EPSRC, 2.12.2008, Übersetzung der Autorin).

Die Vertreterin eines Branchenverbandes beurteilt das fehlende Engagement der Industrie im Bereich der Nanotechnologie als enttäuschend. Sie vertitt die Meinung, dass verschiedene Akteure von der Industrie erwarten, dass sie sich angesichts des öffentlichen Vertrauensverlustes, den sie in der Gentechnikkontroverse erlitten hat, im Bereich der Nanotechnologie proaktiver positioniert; sei es durch eine aktive Kommunikationsstrategie oder durch die Entwicklung einer selbstregulatorischen Maßnahme:

„Nach der Publikation des RS\&RAE-Berichtes machten sich eine Menge unterschiedlicher Parteien Gedanken über Nanotechnologie. Die einzige, die in konsolidierter Form ein bisschen gefehlt hat, ist die Industrie. Die Royal Society ist ein bisschen enttäuscht darüber; auch angesichts der Lektionen, die aus der GMO Debatte hätten gelernt werden sollen." (Nanotechnologieverantwortliche, NIA, 5.11.2008).

Als wichtigste Erkenntnis aus dem Arbeitstreffen ist die Einsicht entstanden, dass die meisten Akteure den Zeitraum, den die Entwicklung und die Inkraftsetzung einer neuen Regulierung benötigt, unterschätzen. Wie der Vertreter des nationalen Forschungsrates EPSRC erläutert, haben sich die beteiligten Akteure dazu entschieden, angesichts der Regulierungslücke einen zeitnah in Kraft zu setzenden freiwilligen Verhaltenskodex zu entwickeln: 
„Das Ergebnis des Arbeitstreffens war die Entscheidung, dass freiwillige Prinzipien, wie ein Kodex hilfreich wären, zusammen mit dem Gefühl, dass die Regulierung langsamer ist, als es sich verschiedene Leute gewünscht hätten." (Nanotechnologiespezialist, EPSRC, 2.12.2008, Übersetzung der Autorin).

Als ein weiteres Ergebnis des Arbeitstreffens ist ein unabhängiges Forum, das Responsible Nano Forum entstanden. Dessen Aufgabe liegt darin, die Zivilbevölkerung unabhängig über die Nanotechnologie informieren. ${ }^{121}$

Der Responsible Nano Code (RNC) wird im Jahr 2008 veröffentlicht. Dieser prinzipienbasierte freiwillige Verhaltenskodex betrifft die Firmen und Organisationen, die an der Forschung, der Entwicklung, der Produktion, dem Handel und dem Vertrieb von nanoskaligen Substanzen und Produkten beteiligt sind. Ziel der lancierenden Organisationen ist es, ein verstärktes Produktlebenszyklusdenken anzuregen. Der Kodex enthält die sieben folgenden übergreifenden Prinzipien: ${ }^{122}$

1) Die Verankerung der nanotechnologiespezifischen Verantwortlichkeit und Entscheidung auf höchster Führungsebene.

2) Die proaktive Einbindung von Anspruchsgruppen.

3) Die Sicherstellung von Arbeitsgesundheits- und Sicherheitsaspekten sowie die Reduktion potenzieller Gesundheits- Umwelt- oder Sicherheitsrisiken eigener Nanoprodukte über den gesamten Produktlebenszyklus.

4) Die Vermeidung darüber hinausgehender sozialer-, Umwelt-, Gesundheits- und ethischer Implikationen durch die eigene unternehmerische Tätigkeit im Bereich der Nanotechnologie.

5) Der proaktive, offene und kooperative Austausch mit Geschäftspartnern und deren Unterstützung in der Anwendung des Kodexes.

6) Die offene und transparente Kommunikation der eigenen unternehmerischen Tätigkeit im Bereich der Nanotechnologie und der ergriffenen Risikomanagementmaßnahmen.

121 Das Responsible Nanoforum wird inzwischen in Matter umbenannt. Es beschreibt sich als ,action tank“ und fokussiert nicht mehr ausschließlich für Nanotechnologie, sondern grundsätzlich für neue Technologien (emerging technologies) (siehe http://www.matterforall.org/about-us/ 5.7.2011). Matter unterhält unter anderem auch die konsumentenorientierte Website nano\&me (siehe http://w ww.nanoandme.org/home/ 7.7.2011).

122 Siehe http://www.responsiblenanocode.org/ (29.6.2011). 
7) Die regelmäßige Information über die Implementierung des Verhaltenskodexes in der eigenen Firma.

Der RNC wird von den verschiedenen in den Nanotechnologiediskurs involvierten Akteuren unterschiedlich bewertet. Grundsätzlich wird die Idee eines selbstregulatorischen Lenkungsansatzes im unregulierten System begrüßt. Verschiedene Interviewpartner bezeichnen seinen Inhalt und die Umsetzung jedoch als dürftig. Die Vertreterin einer Konsumentenschutzorganisation schätzt den RNC als relativ schwache Maßnahme ein:

„Der Responsible Nano Code ist etwas, das Firmen in der Abwesenheit von Regulierung offenbar haben wollten. Aber wir sind besorgt, da es sich um eine sehr stark verwässerte Maßnahme handelt.“ (Nanotechnologieverantwortliche, WHICH, 12.1.2009, Übersetzung der Autorin).

Zudem vertritt sie die Ansicht, dass dieser mit Ausnahme der Einbindung der nachfolgenden Mitglieder der Lieferkette nicht über die für Herstellerfirmen grundsätzlich geltenden rechtlichen Bestimmungen zur Produktsicherheit hinausgeht:

„Der Kodex ist nichts anderes als eine Neuformulierung der bereits vorhandenen gesetzlichen Verpflichtungen. Einige Details gehen weiter, wie beispielsweise der Versuch, die eigenen Kunden frühzeitig in den Prozess einzubinden. Das ist vielleicht eine nützliche Anleitung. Aber ganz sicher kann dies kein Ersatz für eine gesetzliche Regulierung sein. Und in keinster Weise führt er in die Nähe dessen, was wir uns nur schon als Ausgangslage einer solchen Maßnahme erhofft hatten." (Nanotechnologieverantwortliche, WHICH, 12.1.2009, Übersetzung der Autorin).

Da die beiden Finanzpartner verstärkt kommerzielle Interessen vertreten, stellt der Verantwortliche Nanokodex das Produkt eines Kompromisses dar. Wie ein Interviewpartner der lancierenden Royal Society erläutert, stellt der Kodex eine Zwischenposition zwischen den lancierenden Organisationen dar:

„Die Prinzipien des RNC sind durch die Arbeitsgruppe entwickelt worden, in welcher wir lediglich eine der beteiligten Parteien waren. Deshalb war es das Ergebnis eines Kompromisses. Angesichts der Tatsache, dass zwei der beteiligten Gründungspartner mit einem leicht unterschiedlichen Standpunkt starteten, denke ich, kann man verstehen, dass das, was wir erreicht haben, sich irgendwo zwischen diesen beiden Positionen befindet." (Nanotechnologieverantwortlicher, Royal Society, 5.11.2008, Übersetzung der Autorin).

Der befragte Vertreter des EPSRC vertritt die Ansicht, dass selbstregulatorische Lenkungsmaßnahmen wie der Verantwortliche Nanokodex im Kontext der Produkthaftpflicht eine verbindliche Komponente beinhalten. Im 
Schadensfall werden jeweils Verschulden und Entschädigungspflicht eines Unternehmens nach den von ihm vorgängig ergriffenen Vorsorgemaßnahmen beurteilt. Solche betreffen auch die Mitgliedschaft in einem branchenübergreifenden Verhaltenskodex. Daraus entsteht aus seiner Sicht für die betroffenen Firmen die Verpflichtung, freiwillige Kodizes mitzuunterzeichnen. Ansonsten werden Unternehmen als nicht ausreichend vorsorgeorientiert bewertet und im Schadensfall mit einer Haftpflichtklage belangt. Durch diese potenzielle Verbindlichkeit stellt die inhaltliche Ausgestaltung des NRC seiner Ansicht nach einen hart errungenen Kompromiss dar:

„Die Existenz eines freiwilligen Kodex hat durchaus Rechtskraft im Sinne von; wenn sich eine Katastrophe ereignet und die Firma eingeklagt würde, dann würde die Unterzeichnung eines Industriekodexes durchaus als ein starkes Zeichen gewertet, vernünftige Vorsorgevorkehrungen getroffen zu haben. In diesem Sinn nimmt es weniger die Form von weichem als vielmehr diejenige von hartem Recht an und das ist der Grund, weshalb es wirklich viel problematischer ist, etwas auszuarbeiten, das für alle annehmbar ist. Deshalb ist es eine sehr schwierige und sehr delikate Angelegenheit." (Nanotechnologiespezialist, EPSRC, 2.12.2008, Übersetzung der Autorin).

Die Interviews zeigen, dass die in Großbritannien vor dem Hintergrund der Gentechnikkontroverse und den verschiedenen Lebensmittelskandalen verstärkt wahrgenommene Zurückhaltung der Hersteller zu Irritationen bei den Branchenverbänden und den forschungspolitischen Gremien geführt hat. Angesichts des durch den Royal Society-Bericht in Großbritannien prominent lancierten Risikodiskurses wird ein dringender Bedarf an industriellen Vorsorgemaßnahmen gesehen. Der RNC behandelt Nanomaterialien und darin insbesondere die im Gesetz traditionell verankerte Produktverantwortung. Dabei wird der RNC, auch wenn er branchenintern aus Haftpflichtgründen formal annähernd verpflichtenden Charakter hat, inhaltlich als schwache Maßnahme kritisiert.

\subsubsection{Zusammenfassung Großbritannien: ein Regulierungsdefizit und eine Nichtregierungsinitiative}

Die in den britischen Regulierungsdiskurs involvierten Akteure umfassen Vorsorgebehörden, wie die Behörde für Umwelt, Ernährung und Landwirtschaft DEFRA, die Gesundheitsbehörden und die Lebensmittelbehörde. Im Unterschied zu den Vereinigten Staaten, wo die Wahrnehmung einer Regulierungslücke und mangelnder Aktivität der Regierung zu selbstregulatorischen Maßnahmen geführt hat, sind in Großbritannien regierungsex- 
terne Akteure aus der Wahrnehmung eines mangelnden Engagements der Industrie aktiv geworden. Akteure, wie die wissenschaftliche Akademie Royal Society, die Investitionsberatungsfirma Insight Investment, der neu gegründete Branchenverband NIA und das vom DTI finanzierte Nanotechnology KTN haben sich zusammengeschlossen, um einen prinzipienbasierten Verhaltenskodex zur verantwortungsvollen Nanotechnologieentwicklung zu entwickeln. Während die Royal Society im Abschätzungsdiskurs mit ihrer umfassenden Technikanalyse viel Aufmerksamkeit und $\mathrm{Zu}$ stimmung erhalten hat, ist sie mit ihrem Engagement in der Entwicklung eines freiwilligen Nanokodex weniger erfolgreich gewesen. Dies deutet auf mögliche Schwierigkeiten hin, welche die Entwicklung substanzieller selbstregulatorischer Maßnahmen durch ihre Freiwilligkeit, Unverbindlichkeit und die fehlende Funktionstrennung zwischen der regulierenden Instanz und derjenigen, auf die sich die Maßnahme bezieht, mit sich bringt.

Thematisch stehen in Großbritannien insbesondere die Umwelt-, Gesundheits- und Sicherheitsimplikationen von Nanomaterialien im Fokus des Regulierungsdiskurses. Darin wird, wie in den anderen untersuchten Ländern und auf der Unionsebene, die Frage nach der Behandlung von Nanomaterialien im bestehenden Recht thematisiert und ein Regulierungsdefizit festgestellt.

Die beobachteten Praktiken umfassen das von der DEFRA lancierte freiwillige Datenmeldeschema. Ein solches ist im nachfolgenden Jahr in einer leicht angepassten Form in den Vereinigten Staaten eingeführt worden. Die Rückmeldungen sind, wie bei demjenigen in den Vereinigten Staaten unter den Erwartungen geblieben. Zentrale Defizite des Schemas werden in seiner Freiwilligkeit und in seiner Komplexität gesehen, die insbesondere kleine und mittelgroße Unternehmen und Neugründungen vor einer Teilnahme abgeschreckt haben. Damit gilt der den freiwilligen Datenmeldeverfahren zugrunde liegende Anspruch als gescheitert, Firmen dazu zu bringen, den Behörden auf freiwilliger Basis vertrauliche Daten zur Verfügung zu stellen.

Wie in den Vereinigten Staaten lässt sich auch in Großbritannien die Praxis beobachten, dass ansonsten kaum kooperierende regierungsexterne Akteure selbstregulatorische Ansätze entwickeln. Im Unterschied zum handlungsorientierten DuPont-EDF-NanoriskFramework, das von einem Hersteller mitentwickelt worden ist, wird mit dem Royal Society - Insight Investment-Responsible Nano Code ein prinzipienbasierter Verhaltenskodex von nicht in die Herstellung von Nanomaterialien involvierten Akteu- 
ren publiziert. Während einige Akteure das EDF - DuPont Nanorisk Framework grundsätzlich wohlwollend bewerten, wird der Responsible Nanocode fast durchwegs kritisiert. Dabei dominiert die Einschätzung, dass letzterer mit seiner Prinzipienbasierung, dem breiten Fokus und seinen auf allgemeiner Ebene verhafteten Formulierungen das Ziel kaum erfüllt, in der Abwesenheit einer gesetzlichen Regulierung, Nanotechnologierisiken vorsorgeorientiert zu erkennen. Vielmehr akzentuiert sich am Responsible Nanocode eine grundsätzliche Kritik an selbstregulatorischen Lenkungsmaßnahmen. Diese stellen nach Ansicht von verschiedenen Interviewpartnern häufig ein eher allgemein formuliertes Produkt eines Kompromisses dar, der kaum über die gesetzlich verlangte Produktverantwortung hinausgeht, auch wenn er innerhalb der Branche aus Gründen der Haftpflicht durchaus bindenden Charakter entwickeln kann.

\subsection{Deutschland: Evaluationen des Stoffrechts und selbstregulierende Hersteller}

Vergleichbar mit den anderen untersuchten Ländern wird auch im deutschen Regulierungsdiskurs die Frage nach der Behandlung von Nanomaterialien im Stoffrecht zentral verhandelt. Im Unterschied zu Großbritannien und den Vereinigten Staaten mit ihren freiwilligen Datenmeldeverfahren und der Europäischen Union mit dem Verhaltenskodex für verantwortungsvolle Nanotechnologieforschung, haben die deutschen Behörden keine eigenen nicht bindende regulatorische Maßnahmen ergriffen. Zudem hat es sich gezeigt, dass im Regulierungsdiskurs Deutschlands keine einzelne Maßnahme herausragt. Vielmehr haben Hersteller, Branchenverbände und letztere auch in Kooperation mit Behörden eine Vielzahl an selbstregulatorischen Steuerungs- und Lenkungsmaßnahmen ergriffen, die und dies ist eine weitere Besonderheit Deutschlands - hauptsächlich von der Industrie; also Herstellerfirmen und ihrem Branchenverband, dem Verband der Chemischen Industrie e.V. (VCI), entwickelt worden sind. Die deutschen Behörden haben keine mit den Datenmeldesystemen Großbritanniens und den Vereinigten Staaten vergleichbaren freiwilligen Regulierungsmaßnahmen ergriffen und sind lediglich in Kooperationen mit Firmen und Branchenverbänden in solche eingebunden. Dennoch werden sie im Unterschied zu den Behörden in den Vereinigten Staaten nicht als zu wenig engagiert kritisiert. Im Gegenteil, sie werden als engagiert wahrgenommen. Im Unterschied zu den Vereinigten Staaten und wie auf der Uni- 
onsebene und in Großbritannien gelten die deutschen Vorsorgebehörden als finanziell ausreichend ausgestattet. Die Finanzierung der Behörden ist im deutschen Regulierungsdiskurs nur dann ein Thema, wenn es um Fragen der Forschungsmittel geht (vgl. Kapitel 2.6.7), ansonsten ist sie kein Thema im Diskurs.

\subsubsection{Nanomaterialien im Stoffrecht}

Die Frage, ob Nanomaterialien durch das geltende Stoffrecht ausreichend reguliert sind, wird in Deutschland hauptsächlich in Abschätzungsstudien behandelt. Beispiele umfassen die von regierungsexternen Experten erstellten Rechtsgutachten und Anhörungen unterschiedlicher Anspruchsgruppen (siehe Kapitel 2.6). Dazu hat das Umweltbundesamt UBA im Mai 2006 ein von ihm der Sonderforschungsgruppe Institutionenanalyse Darmstadt und dem Ökoinstitut in Freiburg in Auftrag gegebenes Rechtsgutachten Nano-Technologien (ReNaTe) veröffentlicht (siehe Kapitel 2.6.6). Das Gutachten umfasst eine Analyse des bestehenden Rechtsrahmens, des Regulierungsbedarfs und der Regulierungsmöglichkeiten auf europäischer und nationaler Ebene (Führ, et al. 2006). Ein Vertreter des Umweltbundesamtes erläutert, wie die Erkenntnisse aus dem ReNaTe-Gutachten in die unterschiedlichen Gremien eingespeist werden. Zudem hat das Gutachten eine legitimierende Basis für die fachlichen Vorschläge seiner Behörde geschaffen:

„Basierend auf dem ReNaTe-Gutachten haben wir das Ministerium darauf hingewiesen, wie aus unserer Sicht die rechtliche Situation mit Nano ist. Wir haben daraus Vorschläge an das Ministerium abgeleitet, die auch in Fragestellungen für den Nanodialog; die Nanokommission einmündeten." (Nanotechnologieverantwortlicher, Umweldbundesamt/UBA, Dessau-Rosslau, 16.9. 2008).

Ein in die Verfassung des Gutachtens involvierter Rechtswissenschaftler zeigt Regulierungslücken im Stoffrecht auf. In Übereinstimmung mit den Verfasserinnen und Verfassern des Rechtsgutachtens der Europäischen Kommission spricht auch er die Problematik der Anwendung der in REACH für herkömmliche Stoffe vorgesehenen massenbasierten Regulierung auf Nanomaterialien an. Diese führt dazu, dass lediglich Nanomaterialien mit einem Produktionsvolumen von über einer Tonne pro Jahr von der REACH Regulierung erfasst werden. Da Nanomaterialien meist in geringeren Mengen produziert werden, sieht er hier ein Problem: 
„Es gibt den Bedarf, ein spezifisches Modul in das Stoffrecht zu implantieren, weil dieses mit seiner Tonnenphilosophie auf die Nanomaterialien nicht passt. Darüber gibt es keinen wirklichen Dissens, auch mit der Industrie nicht.“ (Leitungsmitglied, Sonderforschungsgruppe Institutionenanalyse (sofia), Hochschule Darmstadt, 9.9.2008).

Die Regulierungsanalyse der Europäischen Union beschäftigt auch die deutschen Behörden. Ein Vertreter des Bundesinstituts für Risikobewertung stellt die Einschätzung der Kommission infrage, dass Nanomaterialien durch REACH ausreichend abgedeckt sind. Seiner Ansicht nach ist das Nichtwissen zu groß, um dies abschließend zu beurteilen. Er vertritt zudem die Haltung, dass angesichts des umfangreichen Nichtwissens eine neue Regulierung die Unsicherheit noch verstärken würde:

„Wissenschaftliche Komitees der Europäischen Union sind der Ansicht, dass die aktuelle Regulierung Nanoteilchen mitabdeckt. Man kann das nicht so allgemein sagen. Bisher ist das eine Fall-zu-Fall Entscheidung, weil wir zu wenig wissen. Erst wenn wir die geeigneten Kriterien haben, können wir überprüfen, ob spezielle Regulierungen benötigt werden. Da aber die methodische Unsicherheit groß ist, ist es schwierig, jetzt etwas vorzuschreiben, was der Staat nicht kontrollieren kann. Das würde zu einer großen Rechtsunsicherheit führen." (Leitungsmitglied, Bundesinstitut für Risikobewertung/BfR, Berlin 15.10.2008).

Das hohe Maß des mit Nanotechnologie verbundenen Nichtwissens und die damit verknüpften regulatorischen Schwierigkeiten sind auch Gegenstand einer vom BMBF finanzierten und vom Büro für Interdisziplinäre Nanotechnikforschung (nanobüro) an der TU Darmstadt durchgeführten Studie (siehe Kapitel 2.6.6). Darin weisen die Autoren auf die vielschichtigen Wissenslücken in der regulatorischen Behandlung der Nanotechnologie hin (Lösch, et al. 2008):

„Das Phänomen ,Nanotechnologie' konfrontiert uns auch mit systematischen und - damit - präventiv unaufhebbaren Wissensgrenzen.“ (Lösch, et al. 2008, 4).

Vergleichbar zur Einschätzung der Interviewpartner auf Unionsebene, identifizieren die Autoren die Wissensgrenzen bei den fehlenden Standards, Normen und Testverfahren:

„Eine Vielzahl an Wissensgrenzen und Wissenslücken - z.B. fehlende Standards, Normen und Testverfahren, etc. - stellen Sicherheitsforschungen und Regulierungsmaßnahmen vor besondere Herausforderungen." (Lösch, et al. 2008, 4). 
Die Autoren schildern die Nanotechnologie als hoch komplex und als verschiedene Anwendungsbereiche vielschichtig durchdringend dar. Durch die umfassenden Sicherheitsansprüche, die an die Produkte und die Verfahren gestellt werden, schätzen die Autoren diese deshalb als kaum regulierbar ein. Aus diesem Grund schlagen sie ein alternatives Risiko- und Regulierungsmanagementgremium vor (Gammel, et al. 2009); eine Empfehlung, die bis zum Abschluss dieser Studie jedoch nicht umgesetzt worden ist. Zudem lässt sich beobachten, dass sich die Behörden bei Regulierungsfragen stark am europäischen Recht orientieren. Statt eigene freiwillige Rechtsakte zu etablieren, wie das britische Datenmeldeerfahren VRS, konzentrieren sich die deutschen Behörden auf Fragen der Forschung und der gesetzlichen Anpassung von REACH auf Nanomaterialien.

Zudem lässt sich eine enge Kooperation der drei Vorsorgebehörden Umweltbundesamt (UBA), Bundesinstitut für Risikobewertung (BfR) und Bundesanstalt für Arbeitsschutz und Arbeitsmedizin (BAuA) sowohl in der Forschung als auch in Regulierungsfragen feststellen. Aus dieser Kooperation entstehen unter anderem zwei gemeinsame Positionspapiere zu Forschungs- und Regulierungsstrategien in den Bereichen Umwelt, Sicherheit und Gesundheit (siehe Kapitel 2.6.7). Ein Verantwortlicher des Bundesinstituts für Risikobewertung (BfR) erläutert, dass seine Behörde den effektivsten Regulierungsweg in der Anpassung der Europäischen Chemikalienregulierung REACH sieht. Er zeigt die regulatorischen Herausforderungen auf, Stoffe sowohl hinsichtlich ihrer chemischen als auch ihrer physikalischen Eigenschaften zu beurteilen:

„In REACH ist die Nanoskaligkeit nicht explizit erwähnt, aber der ,physical state' bei der Exposition. Das gibt die Möglichkeit, nanoskalige Eigenschaften miteinzubeziehen. Wir schlagen vor, dass explizit auf die Größe und die Charakteristika der Nanoteilchen eingegangen wird. Das sind physikalische Eigenschaften und das Stoffrecht ist ein Chemikalienrecht. Deshalb beurteilen wir jetzt unter REACH beides; die stofflichen Eigenschaften und allfällige Reaktionen aufgrund der Nanoskaligkeit des Stoffes." (Leitungsmitglied, Bundesinstitut für Risikobewertung/BfR, Berlin 15.10.2008).

In ihrem zweiten, im Jahr 2013 veröffentlichten Positionspapier, schlagen die Vorsorgebehörden ein Konzept vor, wie Nanomaterialien mit ihren spezifischen Eigenschaften mit der REACH-Verordnung wirksam reguliert werden könnten. Diese Änderungen betreffen inbesondere die Anhänge und darin die Empfehlung, die Tonnagegrenzen für die Prüf- und Informationspflichten von Herstellern und Importeuren zu reduzieren (BAuA, 
et al. 2013). ${ }^{123}$ Dazu erläutert der Interviewpartner des BfR, dass aus ihrer Sicht die geringen Produktionsvolumina von Nanomaterialien die Anpassung der Tonnagegrenze in REACH notwendig machen:

„REACH gilt erst ab einer Tonne [Produktionsvolumen] pro Jahr. In der Nanoteilchenchemie brauche ich nur ganz geringe Mengen, um Effekte zu erzielen. Deshalb muss REACH geändert werden.“ (Leitungsmitglied, Bundesinstitut für Risikobewertung/BfR, Berlin 15.10.2008).

Wie es sich in den anderen untersuchten Ländern gezeigt hat, spielen im Bereich Arbeitssicherheit auch in Deutschland Grenzwerte eine zentrale Rolle. Ein Verantwortlicher dieser Behörde erläutert, dass die Erarbeitung einer Empfehlung in Kooperation mit den unterschiedlichen Anspruchsgruppen stattfindet. Ebenfalls als bedeutsam für die deutschen Behörden erwähnt er die internationale Kooperation mit Partnerbehörden, die durch die Publikation von Forschungsstrategien begünstigt wird:

„Ein wichtiges Thema für uns sind verbindliche Grenzwerte. Dazu erarbeiten wir im AGS [Ausschuss für Gefahrstoffe] eine Empfehlung. Diese wird in der Regel übernommen, denn dort finden die Anspruchsgruppendiskussionen statt. Da wir unsere Forschungsstrategie international publiziert haben, werden wir als Institution bekannt und tauschen uns mit verschiedenen Arbeitsschutzbehörden aus, wie derjenigen Brasiliens und Australiens." (Nanotechnologieverantwortlicher Bundesanstalt für Arbeitsschutz und Arbeitsmedizin/ BAuA, 18.9.2008).

Die internationale Kooperation ist auch für weitere Vorsorgebehörden wichtig. Der Vertreter des Bundesinstituts für Risikobewertung weist darauf hin, dass seine Behörde den Regulierungsbedarf in Zusammenarbeit mit Amtskollegen aus anderen Mitgliedsstaaten der Europäischen Union ermittelt. Zudem dient die internationale Kooperation auf der OECD-Ebene der Überprüfung von Testvorschriften:

„Zusammen mit Kollegen aus England und den Niederlanden, haben wir den Regulierungsbedarf ermittelt. ${ }^{124}$ Ich denke, wir müssen abwarten. Im Rahmen

123 Die Behörden sehen die Verbesserung der Informationsübermittlung im Sicherheitsdatenblatt vor, sowie eine über die Stoffregistrierung hinausgehende risikobezogene Behandlung der Nanomaterialien auch bei der Dossier- und Stoffbewertung und in eventuellen Zulassungs- und Beschränkungsverfahren. Die Behörden empfehlen auch die gemeinsame Registrierung von nanoskaligen Formen mit dem chemisch identischen Basismaterial sowie die eigenständige Bewertung und Dokumentation der damit verbundenen Risiken (BAuA, et al. 2013).

124 Dies erfolgt im Rahmen einer akademischen Publikation (Chaudhry, et al. 2010). 
der OECD prüfen wir, ob unsere Testvorschriften ausreichen." (Leitungsmitglied, Bundesinstitut für Risikobewertung/BfR, Berlin 15.10.2008).

Im Unterschied zu Großbritannien, den Vereinigten Staaten und der Europäische Union haben die deutschen Behörden keine freiwilligen Regulierungsmaßnahmen ergriffen. Die Strategien der Behörden im Umgang mit dem mit der Nanotechnologieregulierung verbundenen Nichtwissen umfassen das Verfassen von Abschätzungsstudien und Positionspapieren zur Nanotechnologietauglichkeit des europäischen Rechts, wie insbesondere $\mathrm{REACH}$, die internationale Kooperation mit Partnerbehörden und die nationale Zusammenarbeit mit unterschiedlichen Anspruchsgruppen. Neben den vielfältigen informellen Aktivitäten der Behörden zeigt sich in Deutschland auch eine ausgeprägte Tendenz der Selbstregulierung durch regierungsexterne Akteure. Diese ist Gegenstand des nachfolgenden Kapitels.

\subsubsection{Selbstregulierung durch Hersteller}

Selbstregulatorische Steuerungs- und Lenkungsansätze werden in Deutschland hauptsächlich von den Industrieunternehmen und ihren Branchenverbänden ergriffen. Dabei fällt insbesondere die hohe Anzahl der ergriffenen Maßnahmen auf und dass in Deutschland hauptsächlich die Industrie, also Herstellerfirmen und der Branchenverband VCI besonders aktiv in der Entwicklung solcher Ansätze sind. In Deutschland hat - im Unterschied beispielsweise zu Großbritannien und in diesem hohen Ausmaß auch zu den Vereinigten Staaten - hauptsächlich die zentral in die Produktion von nanoskaligen Materialien und Produkten involvierte Branche, die chemische Industrie und die ihr zugewandten Bereiche, angefangen, sich selbst zu regulieren. Dies ist zeitlich vergleichsweise früh geschehen, nämlich bereits im Jahr 2004. Einzelne, insbesondere multinational operierende Konzerne haben Sicherheitsleitfäden, Verhaltenskodizes oder nanotechnologiespezifische Risikomanagementsysteme erarbeitet. Solche Maßnahmen sind auch in Kooperation mit Branchenverbänden entwickelt worden. Die Branchenverbände wiederum haben einzelne Maßnahmen auch in Kooperation mit den Behörden verfasst. Beispielsweise hat der Verband der chemischen Industrie e.V. (VCI) im Jahr 2004 zusammen mit dem multinationalen Chemie-Unternehmen BASF einen ersten Verhaltenskodex zum sicheren Umgang mit Nanomaterialien am Arbeitsplatz publiziert (vgl. Fleischer 2011). Eine Industrievertreterin führt die 
selbstregulatorischen Aktivitäten der chemischen Industrie Deutschlands auf eine verantwortungsbewusste Haltung zurück, die sie in dieser Branche bereits seit längerer Zeit beobachtet:

„Das ist nichts wirklich Neues, letztendlich hat die chemische Industrie immer schon Responsible Care, oder sagen wir seit 25 oder 30 Jahren." (Nanotechnologieverantwortliche, Corporate \& Governmental Relations, BASF Deutschland, 11.09.08).

Zudem haben sowohl der VCI als auch die Firma BASF im Jahr 2006 Empfehlungen zum Umgang mit Nanomaterialien veröffentlicht. Während sich die Empfehlungen des VCI hauptsächlich auf eigene Positionen und rechtliche Gesichtspunkte konzentrieren (VCI 2006), schlägt BASF in seinem Arbeitssicherheits-Leitfäden konkrete Schutzmaßnahmen und die Verwendung geschlossener Systeme für die Arbeitsplätze mit Nanomaterialien vor (BASF 2006). Aus einer Zusammenarbeit zwischen dem VCI und der Bundesanstalt für Arbeitsschutz und Arbeitsmedizin (BAuA) sind weitere Maßnahmen, wie die handlungsorientierten BAuA-VCI-Leitfäden für Tätigkeiten mit Nanomaterialien am Arbeitsplatz entstanden (BAuA \& VCI 2007; BAuA \& VCI 2012), eine gemeinsame Erhebung unter Mitgliederfirmen über Arbeitssicherheits- und Gesundheitsaspekte von synthetischen Nanomaterialien, Sicherheitsdatenblätter für Laborarbeiten mit Nanomaterialien sowie ein Publikationsplan für verschiedene Studien. ${ }^{125}$ Eine Branchenvertreterin erläutert, dass auf der Verbandsebene lancierte selbstregulatorische Ansätze branchenübergreifend sichtbar werden. Diese Sichtbarkeit ist angesichts des übergreifenden Charakters der Nanotechnologie von zentraler Bedeutung; insbesondere auch deshalb, weil sie in anderen Branchen außerhalb der Chemie kaum Aktivitäten sieht:

„Letztendlich kann so was nur der VCI machen. Deswegen haben wir das auch in den VCI eingebracht. Denn bisher ist fast nur die chemische Industrie aktiv. Es gibt noch ein paar andere Branchen, aber einige sind außen vor und wir haben versucht, das ein bisschen breiter aufzustellen." (Nanotechnologieverantwortliche, BASF Deutschland, 11.09.08).

Die Kooperation mit einem Branchenverband bietet den Behörden den Vorteil des direkten Datenzugangs. Zudem zeigen sich die Branchenver-

125 Die BAuA-VCI-Leitfäden empfehlen eine Reihe von Schutzmaßnahmen für das Arbeiten mit Nanomaterialien und liefern methodische Hinweise für die Gefährdungsbeurteilung von Nanomaterialien am Arbeitsplatz.Siehe dazu beispielsweise https://www.vci.de/Themen/Chemikaliensicherheit/Nanomaterialien/Seiten/Li stenseite.aspx/ (17.04.12). 
bandsvertreter verantwortlich dafür, den Datentransfer zu den Behörden sicherzustellen. Ein Vertreter des VCI bewertet die Weitergabe von Wissen als die zentrale Aufgabe seines Verbandes. Dabei schildert er, wie sein Verband in einer konstruktiven Zusammenarbeit mit den Behörden einen Leitfaden zum Arbeitsschutz erarbeitet hat:

„Das ist unsere Verantwortung, dieses Wissen weiterzugeben. Manches arbeiten wir in Kooperation mit den Behörden aus, zum Beispiel den Leitfaden zum Arbeitsschutz. Wir arbeiten gerne mit den Behörden. Die sind in der Regel kompetent, weil sie ja auch Fachleute sind. So gibt es eine Gruppe, die REACH für Nano umsetzen soll und da haben wir Vorschläge zur Ausgestaltung des Sicherheitsdatenblatts eingereicht." (Nanotechnologieverantwortlicher, Verband der Chemischen Industrie e.V. (VCI), 3.9.2008).

Im Anschluss haben weitere Hersteller selbstregulatorische Maßnahmen veröffentlicht, wie beispielsweise der Pharmakonzern Bayer, der im Jahr 2007 einen Leitfaden für die gute Praxis (Code of Good Practice) zum Umgang mit Nanomaterialien entwickelt hat (Bayer 2007). Ein Interviewpartner der Europäischen Kommission begrüßt die privaten Initiativen der deutschen Industrieunternehmen, selbstregulatorische Ansätze zu entwickeln:

„In Deutschland haben BASF und Bayer eigene, ziemlich weitgehende Initiativen entwickelt, die unabhängig von nationalen oder auch internationalen Behörden geschehen. Und es ist auch sinnvoll, dass sie das für sich selbst machen. Das ist im Sinn von direkter Regulierung der Industrie." (Nanotechnologieverantwortlicher, Europäische Kommission, DG Research, 7. Oktober 2008).

Weitere selbstregulatorische Steuerungs- und Lenkungsmaßnahmen folgen in den Jahren 2008-2010. Diese umfassen beispielsweise die Sicherheitsempfehlung der deutschen Berufsgenossenschaft Rohstoffe und chemische Industrie (BG RCI/damals BG Chemie) sowie die vom Chemieunternehmen Evonik verfassten prinzipienorientierten Strategien des unternehmensinternen Umgangs mit Nanomaterialien und seine Deklaration, dass es dem International Council of Chemical Associations (ICCA) Einsicht in seine Daten gewährt. ${ }^{126}$

126 Diese Deklaration umfasst eine Kurzzusammenfassung über die betriebseigene Verwendung von Nanomaterialien, Arbeitssicherheits- und Umweltschutzvorkehrungen, Informationen $\mathrm{zu}$ betriebseigenen Risikoforschungsansätzen und zur Umsetzung der Anforderungen unter der Europäischen Chemikalienregulierung REACH (siehe http://nanotech.law.asu.edu/ 7.6.2011). 
Die zu einem späteren Zeitpunkt im Diskursverlauf ergriffenen Lenkungsmaßnahmen stehen in einem verstärkten Bestreben nach internationaler Zusammenarbeit. Ein Branchenvertreter erläutert, dass die Selbstverpflichtung in der Chemiebranche auch auf der internationalen Ebene vorangetrieben wird. Dabei nimmt die internationale Kooperation in den entsprechenden Branchenverbänden eine zentrale Stellung ein. Themen und Praktiken der internationalen Kooperation umfassen vergleichbar zur nationalen Ebene, Gesundheits-, Sicherheits- und Umweltfragen, Öffentlichkeitsarbeit, Wissensaustausch und Kooperation mit den Regierungsorganisationen:

„Als Gründungsunternehmen haben wir in der ICCA an der weltweiten Selbstverpflichtung der chemischen Industrie, mitgewirkt. Hier geht es darum, Sicherheit, Gesundheit und Umweltschutz bei der Entwicklung, Herstellung und Verwendung von Produkten zu verbessern, das Wissen über die Auswirkungen auf Mensch und Umwelt zu erweitern, das Bewusstsein der Mitarbeiter für Sicherheit und Umweltschutz zu stärken, und Abnehmer, Weiterverarbeiter, Anwender und die Öffentlichkeit über die sichere Anwendung $\mathrm{zu}$ informieren, in den Dialog zu treten und mit Regierungen und Behörden bei der Entwicklung und Umsetzung von wirksamen Vorschriften zusammenzuarbeiten." (Nanotechnologieverantwortlicher, Evonik/Degussa, HanauWolfgang, 10.9.2008).

Weitere Maßnahmen umfassen beispielsweise das Positionspapier zur Arbeitssicherheit der Deutschen Gesetzlichen Unfallversicherung (DGUV) und die im gleichen Jahr veröffentlichten Antworten auf häufig gestellte Fragen zu Gesundheitsaspekten (DGUV 2010). In der Folge hat die DGUV gemeinsam mit Berufsgenossenschaften, wie der BG Chemie, Hilfestellungen für den Umgang mit Nanomaterialien im Labor publiziert (DGUV 2012). Zudem hat der Verband der deutschen Lack- und Druckfarbenindustrie e.V. (VDL) einen Leitfaden für den Umgang mit Nanomaterialien am Arbeitsplatz entwickelt (VDL 2010).

In der Analyse des deutschen Regulierungsdiskurses stellt sich die Frage, weshalb die Herstellerfirmen und die Branchenverbände im Unterschied zu den anderen untersuchten Ländern derart aktiv die Entwicklung selbstregulatorischer Ansätze vorangetrieben haben. Dazu werden in den Interviews insbesondere zwei Aspekte angesprochen, welche die Haltung der deutschen Chemieindustrie angesichts des mit potenziellen Implikationen der Nanotechnologie verknüpften Nichtwissens beeinflusst haben. Die Aktivität der deutschen Industrie im Nanotechnologiediskurs mit früheren Technikkontroversen verglichen, wie beispielsweise der Gentechnik. Damals ist die Industrie für ihre intransparente Kommunikationspolitik kriti- 
siert und als mitverantwortlich für die ablehnende Haltung der Öffentlichkeit bezichtigt worden. Zum anderen wird der Industrie grundsätzlich eine neoliberale Regulierungsaversion unterstellt, die im Fall der Nanotechnologie zu Rechtsunsicherheit insbesondere in Haftungsfragen führt. Dazu erläutert ein Interviewpartner der Arbeitsschutzbehörde, dass die Industrie aus der Gentechnikkontroverse die Lehre gezogen hat, dass mit proaktivem Verhalten und transparenter Information das öffentliche Vertrauen gewonnen und eine strikte gesetzliche Regulierung vermieden werden kann:

„Im Bereich genetisch modifizierte Organismen hat sich gezeigt, dass durch mangelnde Transparenz in der Öffentlichkeit ein Misstrauen entstanden ist, was zu strengen Gesetzen geführt hat. Da hat die Industrie gesagt, hier müssen wir mit Informationen an die Öffentlichkeit gehen und die Kooperationen mit Behörden suchen, um Vertrauen und Transparenz zu schaffen. Wie weit das dann wirklich ausreicht, das muss man sehen. Aber zunächst einmal bin ich mit der Industrie recht zufrieden." (Nanotechnologieverantwortlicher Bundesanstalt für Arbeitsschutz und Arbeitsmedizin/BAuA, 18.9.2008).

Zudem wird die hohe Zahl der in Deutschland entstandenen industriellen Selbstregulierungsmaßnahmen auch auf den Nachahmungseffekt zurückgeführt. Dazu vertritt der Interviewpartner eines privatwirtschaftlichen Forschungs- und Beratungsinstituts die Meinung, dass individuelle Selbstregulierungsmaßnahmen einelner Firmen zu einer Selbstregulierungsdynamik in der gesamten Branche geführt haben:

„Wenn BASF so etwas [Code of Conduct] macht, dann wird Bayer auch gefragt, was sie machen. Das ist eine andere Regulierungsdynamik, die da entstehen kann." (Nanotechnologiespezialist, Institut für Ökologische Wirtschaftsforschung/IÖW, Berlin, 15.10.2008).

Aus den in früheren Kontroversen, wie denjenigen um die Gentechnik festgestellten kommunikationsstrategischen Defiziten der Industrie wird für diese im Nanotechnologiediskurs die Notwendigkeit abgeleitet, kommunikativ aktiv zu werden. Bei verschiedenen Interviewpartnern scheint Konsens darin zu bestehen, dass mit einer frühzeitigen und transparenten Informations- und Selbstregulierungsstrategie die Entwicklung einer kritischen Haltung in der Öffentlichkeit und kontroverse Diskurse verhindert werden können. Diese Auffassung entsteht auch aus der Überzeugung, dass eine vorausschauende Kommunikationsstrategie eine vertrauensbildende Maßnahme darstellt, mit welcher die Industrie die öffentliche Wahrnehmung beeinflussen kann. Die Notwendigkeit zur Kommunikation wird mit den Attributen der Proaktivität, Transparenz und Verantwortung - 
bzw. im nachfolgenden Interviewzitat des IÖW-Vertreters mit ,Sensibilität' — umschrieben:

„Das Biotechnologieargument ist schon früh in der Diskussion gewesen. Die Industrie hat gesehen, dass Technologien nicht mehr nur als gut bewertet werden. So müssen sie in dem Bereich [der Nanotechnologie] sensibler handeln.“ (Nanotechnologiespezialist, Institut für Ökologische Wirtschaftsforschung/ IÖW, Berlin, 15.10.2008).

Daneben zeigt sich auch die Überzeugung, dass eine transparente Kommunikation nicht nur die gesellschaftliche Akzeptanz fördert, sondern darüber hinaus direkt den ökonomischen Erfolg beeinflusst. Ein Branchenvertreter erläutert, dass für seine Organisation der Dialog mit den Anspruchsgruppen strategisch wichtig ist:

„Die auf der Nanotechnologie basierenden Produkte werden sich nur dann dauerhaft durchsetzen, wenn die Gesellschaft sie akzeptiert und ihren Nutzen erkennt. Deswegen verpflichten wir uns zu einem verantwortungsvollen Umgang mit der Nanotechnologie. Dazu gehört das Informieren und das umfassend in den Dialog Treten mit den beteiligten interessierten Gruppen als ein notwendiger Aspekt einer erfolgreichen Geschäftsstrategie." (Nanotechnologieverantwortlicher, Evonik/Degussa, Hanau-Wolfgang, 10.9.2008).

Ein weiterer Branchenverbandsvertreter sieht diese Notwendigkeit im Ergreifen von selbstregulatorischen Ansätzen erfüllt. Da er potenzielle, mit der Nanotechnologie verbundene Risiken als gering einschätzt, bewertet er die Selbstregulierung eher als Reputationspflege, als dass er ihr eine substantielle Vorsorgewirkung abgewinnt:

„Ich sehe es als Goodwillmaßnahme der Industrie, was besser ist, als wenn sie sich abschotten würde. Auch wenn das Risiko eher als gering eingeschätzt wird, so kann es durch die Informationspolitik durchaus negativ gewertet werden. (Wolfgang Luther, VDI Technologiezentrum, Düsseldorf, 9.9.2008).

Ein problematischer Aspekt der industriellen Selbstregulierung wird in der geforderten Transparenz gesehen. So kann diese zu Interessenskonflikten führen, da die unverschlüsselte Kommunikation sensibler Daten zwar der Vertrauensbildung dient, jedoch auch zu kompetitiven Benachteiligungen und kommerziellen Einbussen führen kann. Der Interviewpartner des VDI-Technologiezentrums spricht diese Problematik an. Er sieht die Grenzen von freiwilligen Maßnahmen dann als erreicht an, wenn vertrauliche Daten transparent gemacht werden müssen. Dort entstehen Interessenskonflikte um die Innovationsvorherrschaft, die nicht alleine bei den selbstregulatorischen Steuerungs- und Lenkungsmaßnahmen auftreten, sondern auch im Kontakt mit den Behörden eine Rolle spielen: 
„Bei diesen freiwilligen Maßnahmen spielen immer auch wettbewerbsrechtliche Aspekte eine Rolle. Wenn man eine Risikoabschätzung abgeben möchte, muss man technisch relevante Informationen preisgeben und das ist ein Problem, das auch meiner Einschätzung nach nicht leicht zu lösen ist. Auch in einem geschützten informellen Datenaustausch mit den Behörden sind immer verschiedene Interessen abzuwägen, also Firmeninteresse und öffentliche Interessen." (Nanotechnologieverantwortlicher, VDI Technologiezentrum, Düsseldorf, 9.9.2008).

Die Entwicklung von selbstregulatorischen Ansätzen durch regierungsexterne Organisationen und Akteure wird in den Interviews unterschiedlich bewertet. Einerseits werden ihre Verbindlichkeit und ihr Nutzen insbesondere im Schadensfall angezweifelt. Andererseits werden informelle Ansätze in Bereichen umfangreichen Nichtwissens im Vorregulierungsdiskurs als gewinnbringend bewertet. Ein Behördenvertreter äußert sich kritisch zur Verbindlichkeit von industrieller Selbstregulierung:

„Wenn freiwillige Maßnahmen von $80 \%$ der Hersteller getragen werden, müsste ich billigend in Kauf nehmen, 20\% meiner Bevölkerung nicht zu schützen und das kann ich natürlich alleine schon aufgrund der gesetzlichen Lage nicht. (Leitungsmitglied, Bundesinstitut für Risikobewertung/BfR, Berlin 15.10.2008).

Derselbe Interviewpartner sieht die Vorteile der freiwilligen Maßnahmen in der Sensibilisierung. Im Schadensfall hingegen, reichen selbstregulatorische Ansätze seiner Ansicht nach nicht aus:

„Freiwillige Massnahmen sind ein guter erster Schritt zur Sensibilisierung, aber sobald es sicherheitsrelevante Belange oder um eine justiziable Überwachung betrifft, reicht Freiwilligkeit nicht aus. Denn mit einer freiwilligen Massnahme können Sie im eventuellen Schadensersatzfall vor Gericht nichts erreichen." (Leitungsmitglied, Bundesinstitut für Risikobewertung/BfR, Berlin 15.10.2008).

Ein Verteter einer deutschen Arbeitsschutzbehörde betont, dass der gesetzliche Weg, angesichts des hohen Maßes an Nichtwissen hinsichtlich der arbeitsmedizinischen Auswirkungen von Nanomaterialien, nicht zielführend ist. Am Beispiel der Entwicklung von Grenzwertvorschlägen in Bereichen mit umfangreichen Wissenslücken erläutert er den Nutzen von informellen Vorgehensweisen. Solche umfassen die Diskussion der eigenen Position in Abschätzungsstudien und in internationalen Gremien, sowie die Publikation von eigenen Erkenntnissen in internationalen Zeitschriften:

„Für Nanomaterialien gibt es kaum Grenzwerte und es wird nach dem Minimierungsprinzip, also einer Annäherung an Null, vorgegangen. Dies ist für 
die Praxis schwierig, die lieber einen konkreten Wert haben will und grosses Interesse an einem Vorschlag hat. Vor diesem Hintergrund macht es Sinn, nicht nur den traditionellen Weg zu wählen, da das im Einzelfall lange dauern kann, sondern eine eigene Position zu beziehen und mit einer Veröffentlichung die internationale Diskussion zu befruchten." (Nanotechnologieverantwortlicher Bundesanstalt für Arbeitsschutz und Arbeitsmedizin/BAuA, 18.9.2008).

Verschiedene Interviewpartner sehen die selbstregulatorischen Steuerungsund Lenkungsmaßnahmen hauptsächlich als Übergangsstadien in der Einführungsphase von neuen Technologien an. Diese sind nach Ansicht der Vertreterin von BASF insbesondere im Zeitraum vor der Inkraftsetzung einer gesetzlichen Regulierung bedeutsam:

„Der Code of Conduct kam aus der Politik heraus. Wir haben vor vier Jahren gesehen, dass Fragen zum sicheren Umgang mit Nanomaterialien aufkommen, die man nicht beantworten kann, obwohl bereits Produkte auf dem Markt sind. Diese Situation braucht einen Rahmen. Die Politik kann nicht auf Grund von Annahmen regulativ tätig werden, das ist nicht durchsetzbar. Das kann nur mit Hilfe freiwilliger Instrumente klappen. Deswegen haben wir diesen Code of Conduct gemacht." (Nanotechnologieverantwortliche, BASF Deutschland, 11.09.08)

Die Herstellervertreterin betrachtet demnach die freiwillige Selbstregulierung und die Kooperation mit der Politik und den Industrieverbänden dann als besonders hilfreich, wenn das Maß an Nichtwissen hoch ist. Der Vorteil solcher Maßnahmen ergibt sich also dann, wenn Produkte vor der Klärung von Sicherheitsfragen und vor dem Vorliegen ausreichender Information für eine gesetzliche Regulierung in den Markt eingeführt werden.

\subsubsection{Zusammenfassung Deutschland: Ein Regulierungsdefizit und selbstregulierende Hersteller}

Die in den deutschen Regulierungsdiskurs eingebundenen Akteure umfassen wie in den anderen untersuchten Ländern und auf der Unionsebene die Vorsorgebehörden der Bereiche Umwelt, Gesundheit, Arbeitssicherheit und Risikovorsorge. Im Unterschied zu Großbritannien und größtenteils auch den Vereinigten Staaten, zeigt sich im deutschen Regulierungsdiskurs die Herstellerindustrie als überwiegend aktive Branche. Daneben spielen ihre Verbände, die Berufsgenossenschaften und die gesetzlichen Versicherer in der Entwicklung von selbstregulatorischen Ansätzen eine aktive Rolle. 
Die Analyse der Themen des deutschen Regulierungsdiskurses zeigt, dass hier ebenfalls die Frage nach der Behandlung von Nanomaterialien im bestehenden Stoffrecht bedeutsam ist. Dabei stellen auch in Deutschland verschiedene Akteure ein Regulierungsdifizit fest. Das vergleichsweise intensive Engagement der deutschen Hersteller wird thematisch mit einem in der Gentechnikkontroverse festgestellten ,Kommunikationsdefizit' der Industrie begründet. Dabei wird die Haltung vertreten, dass eine transparente Kommunikation die gesellschaftliche Akzeptanz neuer Technologien begünstigt. Daraus leiten Herstellerfirmen das Dispositiv ab, kommunikative Aktivitäten und selbstregulatorische Maßnahmen zu entwickeln.

Bezüglich der ergriffenen Praktiken fällt auf, dass die deutschen Behörden kein Dispositiv zur Entwicklung eigener freiwilliger Ansätze abgeleitet haben und keine mit den Vereinigten Staaten, Großbritannien und der Europäischen Union vergleichbaren Maßnahmen ergriffen haben, wie die Entwicklung eines freiwilligen Datenmeldeverfahrens oder die Etablierung eines Verhaltenskodexes. Vielmehr wählen die Vorsorgebehörden hauptsächlich kooperative und konsensorientierte Strategien wie die Mitarbeit in internationalen Gremien, den Wissensaustausch, die Anhörung von Anspruchsgruppen und das Verfassen von gemeinsamen Positionen. Darüberhinaus ist insbesondere die Arbeitssicherheitsbehörde in Kooperation mit den Herstellern in verschiedene selbstregulatorische Steuerungsund Lenkungsansätze involviert. Beispielsweise hat die BAuA gemeinsam mit dem Branchenverband der chemischen Industrie verschiedene Leitfäden und Sicherheitsdatenblätter für Nanomaterialien am Arbeitsplatz entwickelt.

Möglicherweise haben die Aktivitäten der Hersteller nicht nur den Regulierungsdiskurs, sondern auch die regulatorischen Strategien der deutschen Behörden im Hinblick auf die Verteilung der Regulierungskompetenz beeinflusst. Die Behörden haben möglicherweise angesichts der vielfältigen Aktivitäten der Industrie die Haltung entwickelt, dass keine ergänzenden Maßnahmen notwendig sind. Die auch im deutschen Diskurs beobachtete Feststellung einer Regulierungslücke und die in den Interviews abgebildeten kritischen Stimmen, insbesondere die von Behördenvertretern, deuten jedoch darauf hin, dass die Delegation der Regulierungskompetenz an die Hersteller dem Bedürfnis nach klaren Richtlinien nicht zu entsprechen vermag. 


\subsection{Nichtwissen Regulieren: Gesellschaftliche Distribution der Regulierungsverantwortung}

Ein zweites zentrales Dispositiv im wissenschaftspolitischen Diskurs um die Nanotechnologie zeichnet sich durch den länderübergreifenden Ansatz aus, Nichtwissen mittels der Integration in bestehende gesetzliche und neugeschaffene freiwillige und selbstregulatorische Ansätze zu lenken. Dabei sind im Nanotechnologiediskurs neben den traditionellerweise in die Regulierung involvierten nationalen Regierungen und Behörden auch internationale und vielfältige regierungsexterne Akteure, wie Firmen, Umweltorganisationen, Wissenschaftsakademien, Expertengremien und Stiftungen an den Regulierungsaktivitäten beteiligt. Wo nicht gesetzlich reguliert wird, gewinnen diese im Rahmen der in der Literatur unter dem Begriff der ,new governance' bezeichneten Phänomene an Bedeutung und führen zur Distribution der Regulierungsverantwortung an die Gesamtgesellschaft.

Im Unterschied zum Abschätzungsdiskurs zeigt sich der Regulierungsdiskurs thematisch etwas eingeschränkter. Da Regulierungsfragen im Diskursverlauf hauptsächlich nach der Fokusverschiebung von der Nanotechnologie auf Nanomaterialien und von ELSI zu EHS bedeutsam geworden sind, konzentrieren sich diese in erster Linie auf EHS, also Umwelt, Gesundheits- und Sicherheitsfragen von Nanomaterialien. Visionen spielen kaum eine Rolle. Auch soziale und ethische Aspekte spielen eine eher untergeordnete Rolle. Zu letzteren hat lediglich die Europäische Kommission eine Maßnahme zur Sicherstellung der Forschungsverantwortung ergriffen. Wichtige Themen im Regulierungsdiskurs umfassen Fragen der Kooperation und der Harmonisierung ebenso wie solche nach Definitionen, Grenzwerten und der Implementierung der regulatorischen Vorgaben auf Nanomaterialien. Diese Themen spielen in den untersuchten Ländern eine vergleichbare Rolle und werden von den zuständigen nationalen Behörden auch in die Diskussionen in den internationalen Gremien hineingetragen.

Durch das in Nichtwissensdiskursen beobachtbare Dispositiv, die Regulierungsverantwortung an die Gesamtgesellschaft und in Foren der internationalen Kooperation zu delegieren, verlieren im Nanotechnologiediskurs regulierungskulturelle Unterschiede wie Vermeidungs- oder Vorsorgeorientierung zunehmend an Bedeutung. Dabei zeigt die Analyse des Regulierungsdiskurses, dass sich die in der Literatur für die Vereinigten Staaten und Europa beschriebenen regulierungskulturellen Unterschiede der Vermeidungs- versus der Vorsorgeorientierung im Bereich der Nanotech- 
nologie kaum mehr manifestieren. Diese Unterschiede finden sich zwar explizit in den beiden für die regulatorische Behandlung der Nanotechnologie wesentlichen Rechtsakten zum Stoffrecht; im US-amerikanischen Chemikalienrecht TSCA und in der europäischen REACH-Verordnung. Während TSCA als wissenschaftsbasiert, vermeidungsorientiert und schwacher Statut beschrieben wird, gilt REACH als risikobasiert, vorsorgeorientiert und weltweit führendes Statut. Der US-amerikanische Ansatz der Vermeidungsorientierung und der Wissenschaftsbasierung sieht regulatorische Maß-nahmen nur bei Vorliegen eines gesicherten wissenschaftlichen Nachweises eines Schadens vor. In der europäischen Vorsorgeorientierung und Risikobasierung sind regulatorische Maßnahmen auch unter Ungewissheit möglich. Das Ergreifen einer regulatorischen Maßnahme ist in der europäischen Rechtsordnung also bereits beim Verdacht einer potenziellen Schädigung möglich.

Diese divergierenden Regulierungskulturen implizieren, dass sich auch die regulatorische Behandlung der Nanotechnologie in den beiden Wirtschaftsräumen unterscheiden müsste. Das mit den potenziellen nachteiligen Implikationen der Nanotechnologie einhergehende hohe Maß an Nichtwissen hätte in der US-amerikanischen Vermeidungsorientierung die Abwesenheit sämtlicher regulatorischer Akte gerechtfertigt. Demgegenüber hätte die prominente Verankerung des Vorsorgeprinzips in Europa das Schaffen einer gesetzlichen Regulierung von Nanomaterialien gerechtfertigt. Beides lässt sich so nicht feststellen. Vielmehr hat die US-amerikanische Umweltbehörde mit dem freiwilligen Datenmeldeverfahren einen regulatorischen Akt etabliert, der als Akt an sich und trotz der kritischen Rezeption seines Inhaltes und seiner regulatorischen Schlagkraft, als vorsorgeorientierte Maßnahme bezeichnet werden kann. Auf Unionsebene überrascht die angesichts des prominent verankerten Vorsorgeprinzips zurückhaltende regulatorische Behandlung von Nanomaterialien angesichts der verschiedenen Gefährdungshinweise. Das von Böschen \& Wehling (2012) beschriebene Potenzial des Vorsorgeprinzips, neben toxikologisch nachweisbaren Parametern auch Fragen des Nichtwissens, wie Indikatoren für das mögliche Gefährdungspotenzial von Chemikalien zu berücksichtigen, wird in den untersuchten europäischen Ländern und auf Unionsebene nicht ausgeschöpft.

Die kulturraumspezifischen Unterschiede lassen sich hauptsächlich in der Stellung, der Rolle und der Ausstattung der Behörden wiederfinden. Die US-amerikanische Umweltbehörde, die unter den Deregulierungsaktivitäten der zwischen den Jahren 2001 und 2009 regierenden republikani- 
schen Administration marginalisiert worden ist, gilt als finanziell unausreichend ausgestattet. Dieses Problem wird in ihren Partnerbehörden in Großbritannien, Deutschland und bei der Generaldirektion Umwelt auf Unionsebene nicht erwähnt. Bezüglich der finanziellen Ausstattung fällt die Rolle der deutschen Vorsorgebehörden auf, denen im Unterschied zu denjenigen der anderen untersuchten Länder die Kompetenz fehlt, eigene Forschungsmittel einzusetzen. ${ }^{127}$

Kleinere Unterschiede finden sich auch in den Praktiken der jeweiligen Umweltbehörden. So konzentriert sich die US-amerikanische EPA hauptsächlich darauf, wissenschaftliche Analysen zur Sicherheit von Nanomaterialien zu erstellen oder in Auftrag zu geben. Im US-amerikanischen Regulierungskontext der Vermeidungsorientierung wird solch wissenschaftlich gesicherten Daten eine hohe Bedeutung zugewiesen (siehe Kapitel 3.2). Demgegenüber publizieren die britischen und die deutschen Umweltbehörden sowie die Europäische Kommission verstärkt strategische Berichte darüber, wie mit dem Nichtwissen umgegangen werden soll. Sowohl die britischen und deutschen Umweltbehörden als auch die Europäische Kommission führen eigene Rechts- und Technikanalysen durch. Die Behörden aller untersuchten Länder, inklusive der Union, fokussieren im Regulierungsdiskurs auf vergleichbare Bereiche des Nichtwissens, nämlich auf Nanomaterialien, Umwelt-, Gesundheits- und Sicherheitsaspekte (EHS), auf Definitionsfragen von Nanomaterialien und die Frage nach der Zuständigkeit bzw. der Anpassung der bestehenden Gesetze auf die spezifischen Charakteristika von Nanomaterialien und auf die Implementierung der bestehenden Gesetze, wie durch Testrichtlinien, Sicherheitsprüfungen und Grenzwerte.

Im Unterschied zu Deutschland und der Europäischen Union hat Großbritannien ein freiwilliges Datenmeldesystem eingeführt. Dieses gilt als Vorbild für das ein Jahr später in den Vereinigten Staaten eingeführte NMSP. Vergleichbar zu den Praktiken in den Vereinigten Staaten, die Anhänge von TSCA mit neuen Nutzungsregeln (SNUR) zu ergänzen, hat die Europäische Union Nanomaterialien ebenfalls durch die Anpassung des sektoralen Produktrechts in den Bereichen Kosmetika und Lebensmittel und durch die Adaption der die Implikationen betreffenden Anhänge zum Stoffrecht in REACH reguliert. Als weitere vorsorgeorientierte Maßnah-

127 In Deutschland verfügt lediglich das BMBF über Forschungsmittel, auch in thematischen Bereichen, die in die Kompetenz der Vorsorgebehörden fallen, wie Arbeitsschutz, Risikoanalyse und Umweltverträglichkeit. 
men auf Unionsebene können der Ethikkodex für die Nanotechnologieforschung und die Empfehlung zu einer Nanomaterialiendefinition gelesen werden. In Deutschland haben die Behörden keine eigenen, mit denjenigen der Vereinigten Staaten, Großbritanniens und der Europäischen Kommission vergleichbaren nicht bindenden Regulierungsansätze entwickelt. Vielmehr sind freiwillige Ansätze hier insbesondere von der Herstellerindustrie in Form von selbstregulatorischen Maßnahmen ergriffen worden. Dies hat die Behörden möglicherweise in ihren Regulierungsentscheidungen beeinflusst. Dabei umfassen die von den Vorsorgebehörden ergriffenen Ansätze hauptsächlich Studien, Gutachten, Richtlinien, Empfehlungen und eine enge Kooperation untereinander. Dazu kommt die situative $\mathrm{Zu}$ sammenarbeit mit Herstellerorganisationen, wie die Kooperation der Arbeitssicherheitsbehörde BAuA mit dem VCI. Demgegenüber lassen sich in den anderen Ländern keine aus der Kooperation zwischen Behörden und Herstellerorganisationen entstandenen Regulierungsansätze feststellen.

Interessanterweise schätzen die Regierungen in allen untersuchten Ländern die Nanotechnologie als durch die bestehende gesetzliche Regulierung ausreichend abgedeckt ein. Gleichzeitig wird in allen untersuchten Ländern im Bereich der Nanotechnologie ein Regulierungsdefizit festgestellt. Trotz der deutlich strengeren Chemikaliengesetzgebung in der Europäischen Union werden auch dort Regulierungslücken identifiziert. Dies führt sowohl national als auch international zur Mobilisierung von regierungsexternen Akteuren und auf internationaler Ebene auch zu derjenigen von Regierungsorganisationen. Insbesondere in Deutschland haben verschiedene Hersteller vielfältige selbstregulatorische Ansätze entwickelt. In den Vereinigten Staaten und in Großbritannien sind es hauptsächlich regierungsexterne Organisationen, wie Stiftungen, Akademien, Dienstleister und weitere Nichtregierungsorganisationen, die - teils auch in unüblichen Kooperationen - selbstregulatorische Maßnahmen ergreifen.

Wie diese Analyse jedoch zeigt, kranken die freiwilligen und selbstregulatorischen Ansätze an ihrer Unverbindlichkeit und an ihrem unspezifischen und ungeklärten Geltungsbereich. Insbesondere die selbstregulatorischen Maßnahmen sind mit der Schwierigkeit der fehlenden Funktionstrennung zwischen der regulierenden und der von der Maßnahme betroffenen Instanz konfrontiert. Die meisten dieser Maßnahmen sind mit der Absicht entwickelt worden, ein Richtmaß in ihrem Bereich zu setzen und von anderen Firmen und Branchen übernommen zu werden. Das ist in keinem der Fälle in dieser Form eingetreten. Vielmehr haben die von den unter- 
schiedlichen gesellschaftlichen Anspruchsgruppen entwickelten Verfahren $\mathrm{zu}$ einer grundsätzlichen Offenheit geführt, mit welcher verschiedenste Akteure aktiv werden und eigene Maßnahmen entwickeln. Gleichzeitig fühlt sich aber niemand dazu verpflichtet oder verantwortlich, sich ernsthaft mit einer anderen Maßnahme auseinanderzusetzen oder eine solche zu übernehmen.

Damit ist ein Dispositiv der Selbstregulierung entstanden, das zu einer Distribution der Regulierungsverantwortung von nationalen Regierungsorganisationen weg hin zu regierungsexternen Akteuren und in internationale Foren geführt hat. Damit verwischen im Nanotechnologiediskurs regulierungskulturelle Unterschiede, die sich in Bereichen wie in der Umweltund Chemikalienregulierung insbesondere zwischen den Vereinigten Staaten und Europa manifestieren.

Wie beim Abschätzungsdiskurs zeigt sich also auch im Regulierungsdiskurs der Verlust eindeutig zuordenbarer Kompetenzen. Alle beginnen zu regulieren, doch durch die Auflösung der Trennung zwischen der Regulierungsinstanz und dem zu regulierenden Gegenstand, fließen insbesondere in Fällen der Selbstregulierung auch verstärkt Partikularinteressen ein. Diese dürften in einigen Fällen das eigentliche Ziel von Regulierung, nämlich den Schutz von Mensch und Umwelt vor nachteiligen Auswirkungen von Nanomaterialien überlagern. Die unverbindlichen Ansätze führen also zu einer Diffusion der Regulierungsverantwortung in die Gesamtgesellschaft und damit zum Verlust von eindeutig festgelegten institutionellen Kompetenzen und Zuständigkeiten, was auch ihre Durchsetzbarkeit verunmöglicht. Damit verliert die Regulierung unter Nichtwissen also nicht nur ihre Verbindlichkeit, sondern auch ihre Wirkung. 